\title{
A One-Pot Iodo-Cyclization/Transition Metal-Catalyzed Cross-Coupling Sequence: Synthesis of Substituted Oxazolidin-2-ones From $N$-Boc-allylamines
}

\author{
Pauline Chaumont-Olive, ${ }^{1}$ and Janine Cossy ${ }^{1 *}$ \\ ${ }^{1}$ Molecular, Macromolecular Chemistry, and Materials, ESPCI Paris, PSL University, CNRS, \\ 75005 Paris, France.
}

Table of Contents

1. General details 2

2. Substrates synthesis 3

3. lodo-cyclization/metal-catalyzed cross-coupling sequence 13

3.1. Optimization of the iodo-cyclization step 13

3.2. lodo-cyclization/metal-catalyzed cross-coupling sequence $\quad 14$

3.2.1. Iodo-cyclization/cobalt-catalyzed cross-coupling sequence using arylmagnesium bromide

3.2.2. Iodo-cyclization/copper-catalyzed cross-coupling sequence using cyclopropylmagnesium bromide

4. Preparation of the crystals of $3 j$ and 4 and X-Ray crystallographic data 27

5. ${ }^{1} \mathrm{H},{ }^{19} \mathrm{~F}$ and ${ }^{13} \mathrm{C}$ NMR spectra 28 


\section{General Details}

Reagents (Aldrich) were purchased as reagent grade and used without further purification. Reactions were performed in oven-dried glassware under Ar atmosphere. The reactions that require heating were performed using a sand bath equipped with a probe to monitor the right temperature. Flash column chromatography was performed on silica gel ( $60 \AA$, 230-400 mesh, particle size 0.040-0.063 $\mathrm{mm}$, Merck). The solvent compositions are reported individually in parentheses. Analytical thin layer chromatography (TLC) was performed on aluminium sheets coated with silica gel 60 F254 (Merck, Macherey-Nagel) or with silica gel 60 RP-18 $F_{254 s}$ (Merck, Macherey-Nagel). Visualization was achieved using an alkaline aqueous solution of potassium permanganate $\left(\mathrm{KMnO}_{4}\right)$. Evaporation of solvents in vacuo was performed at $25-35{ }^{\circ} \mathrm{C}$ and $900-10$ mbar. Reported yields refer to spectroscopically and chromatographically pure compounds that were dried under high vacuum (0.10.05 mbar) before analytical characterization. ${ }^{1} \mathrm{H},{ }^{13} \mathrm{C}$ and ${ }^{19} \mathrm{~F}$ and nuclear magnetic resonance (NMR) spectra were recorded on a Bruker AV 400 spectrometer at $400 \mathrm{MHz}\left({ }^{1} \mathrm{H}\right), 101 \mathrm{MHz}\left({ }^{13} \mathrm{C}\right)$ and 376 $\mathrm{MHz}\left({ }^{19} \mathrm{~F}\right)$. Chemical shifts $\delta$ are reported in ppm using the residual deuterated solvent signals as an internal reference $\left(\mathrm{CDCl}_{3}: \delta_{\mathrm{H}}=7.26 \mathrm{ppm}, \delta_{\mathrm{C}}=77.16 \mathrm{ppm}\right.$; MeOD: $\left.\delta_{\mathrm{H}}=3.31 \mathrm{ppm}, \delta_{\mathrm{C}}=49.00 \mathrm{ppm}\right)$. For ${ }^{1} \mathrm{H},{ }^{19} \mathrm{~F}$ and ${ }^{13} \mathrm{C} N M R$, coupling constants $J$ are given in $\mathrm{Hz}$ and the resonance multiplicity is described as $s$ (singlet), $d$ (doublet), $t$ (triplet), pent (pentuplet), $m$ (multiplet), and br (broad). All spectra were recorded at $298 \mathrm{~K}$. Infrared (IR) spectra were recorded on a Bruker Tensor $27 \mathrm{FT}$-IR spectrometer and are reported as wavenumbers $v\left(\mathrm{~cm}^{-1}\right)$. High resolution mass spectrometry (HRMS) and analyses were performed by the Laboratoire de Spectrométrie de Masse from Sorbonne Université, Paris using electrospray ionization (ESI) as the mass source and a LTQ-Orbitrap XL from Thermo Scientific (Thermo Fisher Scientific, Courtaboeuf, France) as the mass analyzer. Gas Chromatography coupled to Mass Spectrometry (GC/MS) analyses were performed on a Shimadzu GC/MS-QP2010S using an electronic impact (EI) spectrometer. The abundance indicated for each mass number ( $\mathrm{m} / \mathrm{z}$ values) is given in percentage relative to the strongest peak of $100 \%$ abundance (base peak). Melting points were determined using a Büchi melting point apparatus in open capillaries. Nomenclature follows the suggestions proposed by the software ChemDraw Professional 16.0. 


\section{Substrates synthesis}

\section{General procedure A: $\mathrm{N}$-Boc protection of primary amine}

$\mathrm{Et}_{3} \mathrm{~N}$ (1.5 equiv) and $\mathrm{Boc}_{2} \mathrm{O}$ (1 equiv) were added to a solution of primary amine (1 equiv) in $\mathrm{CH}_{2} \mathrm{Cl}_{2}$ at $\mathrm{rt}$ and the reaction was stirred for $4 \mathrm{~h}$. The reaction mixture was diluted with $\mathrm{CH}_{2} \mathrm{Cl}_{2}$ and washed with $\mathrm{H}_{2} \mathrm{O}(2 \times 10 \mathrm{~mL})$ then brine $(2 \times 10 \mathrm{~mL})$. The organic layer was dried over $\mathrm{MgSO}_{4}$, filtrated and the solvent was evaporated under reduced pressure. The expected compounds were recrystallized in cold hexanes and filtrated, affording white solids.

\section{General procedure B: $\mathbf{N}$-Allylation}

$\mathrm{NaH}$ (1.5 equiv) and allyl bromide (1.5 equiv) were added to a solution of $\mathrm{NH}$-Boc substrate (1 equiv) in DMF at $0{ }^{\circ} \mathrm{C}$. The reaction mixture was warmed up to $60^{\circ} \mathrm{C}$ and stirred for $16 \mathrm{~h}$. After cooling down the suspension to $0{ }^{\circ} \mathrm{C}$, the reaction was quenched with $\mathrm{H}_{2} \mathrm{O}(10 \mathrm{~mL})$ and diluted in AcOEt $(10 \mathrm{~mL})$. The layers were separated and the organic layer was washed with $\mathrm{H}_{2} \mathrm{O}(2 \times 10 \mathrm{~mL})$, a saturated aqueous $\mathrm{NaHCO}_{3}$ solution $(2 \times 10 \mathrm{~mL})$ and brine $(2 \times 10 \mathrm{~mL})$. The organic layer was dried over $\mathrm{MgSO}_{4}$, filtrated and the solvent was evaporated under reduced pressure. The crude product was purified by silica gel column chromatography (petroleum ether/AcOEt) affording the expected compound.

\section{General procedure C: Reduction of carboxylic acid to alcohol}

$\mathrm{BH}_{3} \cdot \mathrm{Me}_{2} \mathrm{~S}$ (2 equiv) was added to a solution of carboxylic acid ( 1 equiv) in THF at $0{ }^{\circ} \mathrm{C}$. The reaction mixture was stirred at $0{ }^{\circ} \mathrm{C}$ for $2 \mathrm{~h}$, warmed up to $\mathrm{rt}$ and stirred for $3 \mathrm{~h}$. The reaction media was cooled down to $0{ }^{\circ} \mathrm{C}$ and slowly quenched with a saturated aqueous $\mathrm{K}_{2} \mathrm{CO}_{3}$ solution $(2 \mathrm{~mL})$. The layers were separated and the aqueous solution was extracted with AcOEt $(2 \times 10 \mathrm{~mL})$. The combined organic layers were dried over $\mathrm{MgSO}_{4}$, filtrated and the solvent was evaporated under reduced pressure. The expected compound was obtained as a pure product without further purification.

\section{General procedure D: Oxidation of primary alcohol to aldehyde}

DMSO (2 equiv) and $\mathrm{Et}_{3} \mathrm{~N}$ (4 equiv) were added to a solution of alcohol (1 equiv) in $\mathrm{CH}_{2} \mathrm{Cl}_{2}$ at $-10{ }^{\circ} \mathrm{C}$. $\mathrm{Py} \mathrm{SO}_{3}$ (2 equiv) was added to the media in three portions over $20 \mathrm{~min}$. The mixture was allowed to warm up to rt and after $16 \mathrm{~h}$ the mixture was treated with citric acid (aq. 10\%, $2 \mathrm{~mL}$ ) and stirred for $10 \mathrm{~min}$. The layers were separated and the organic layer was extracted with AcOEt $(3 \times 2 \mathrm{~mL})$, washed with citric acid (aq. $10 \%, 2 \mathrm{~mL}$ ), with a saturated aqueous $\mathrm{NaHCO}_{3}$ solution $(2 \mathrm{~mL}$ ) and then with brine $(4 \mathrm{~mL})$. The combined organic layers were dried over $\mathrm{MgSO}_{4}$, filtered and the solvent was evaporated under reduced pressure. The crude product was purified by silica gel column chromatography (petroleum ether/AcOEt) affording the expected compound.

\section{General procedure E: Wittig reaction}

$n$-BuLi ( 2 equiv) was added to a solution of methyltriphenyphosphonium bromide (2 equiv) in THF at $-78{ }^{\circ} \mathrm{C}$ affording a yellow suspension. After complete disappearance of the solid (about $1 \mathrm{~h}$ ), a solution of aldehyde (1 equiv) in THF was added dropwise to the media. The reaction mixture was allowed to warm up to $\mathrm{rt}$ and after $16 \mathrm{~h}$ the media was quenched with a saturated aqueous $\mathrm{NH}_{4} \mathrm{Cl}$ solution $(2 \mathrm{~mL})$ and the organic layer was extracted with AcOEt $(2 \times 10 \mathrm{~mL})$. The combined organic layers were dried over $\mathrm{MgSO}_{4}$, filtrated and the solvent was evaporated under reduced pressure. The crude product was purified by silica gel column chromatography (petroleum ether/AcOEt) affording the expected compound.

Note: all the unsatutared amines will be called $N$-Boc, $N$-alkyl allylamines 
Chemical Formula: $\mathrm{C}_{12} \mathrm{H}_{17} \mathrm{NO}_{2}$

$\mathrm{Et}_{3} \mathrm{~N}$ (3.14 mL, $22.5 \mathrm{mmol}, 1.5$ equiv) and $\mathrm{Boc}_{2} \mathrm{O}(3.27 \mathrm{~mL}, 14.3 \mathrm{mmol}, 0.95$ equiv) were added to a solution of benzylamine (1.64 mL, $15 \mathrm{mmol}, 1$ equiv) in $\mathrm{CH}_{2} \mathrm{Cl}_{2}(75 \mathrm{~mL})$ according to General Procedure A. $\mathrm{N}$-Boc-benzylamine 1a' was isolated after recrystallization in cold hexanes as a white solid $(2.93 \mathrm{~g}, 99 \%)$. The spectral data were identical to those reported in the literature.

m.p.: $69^{\circ} \mathrm{C}$;

$\boldsymbol{R}_{\mathrm{f}}=0.24\left(\mathrm{SiO}_{2}\right.$, petroleum ether $\left./ \mathrm{AcOEt}=96: 4\right) ;$

IR (ATR) v = 3339, 3304, 3063, 3033, 2978, 2929, 1702, 1676, 1606, 1537, 1449, 1388, 1364, 1267, $1251,1166,1136,1051,1028,1018,947\left(\mathrm{~cm}^{-1}\right)$;

${ }^{1} \mathrm{H}$ NMR $\left(400 \mathrm{MHz}, \mathrm{CDCl}_{3}\right) \delta$ 7.35-7.24 (m, 5H), 4.89 (br s, $\left.1 \mathrm{H}\right), 4.32(\mathrm{~d}, \mathrm{~J}=5.0 \mathrm{~Hz}, 2 \mathrm{H}), 1.47(\mathrm{~s}, 9 \mathrm{H})$;

${ }^{13} \mathrm{C}$ NMR (101 MHz, $\left.\mathrm{CDCl}_{3}\right) \delta 156.0,139.0,128.7$ (2C), 127.6, $127.4(2 \mathrm{C}), 79.6,44.8,28.5$ (3C);

MS (+EI) m/z: 207 (M+*, 17), 151 (26), 150 (28), 133 (27), 132 (9), 107 (47), 106 (100), 105 (26), 104 (21), 91 (61), 89 (6), 79 (44), 78 (19), 77 (37), 74 (7), 65 (16), 63 (7), 59 (35), 57 (55), 56 (51), 55 (19), $53(9), 52(10), 51(28), 50(18)$.

$N$-Boc, $N$-benzyl-allylamine, $1 a^{2}$

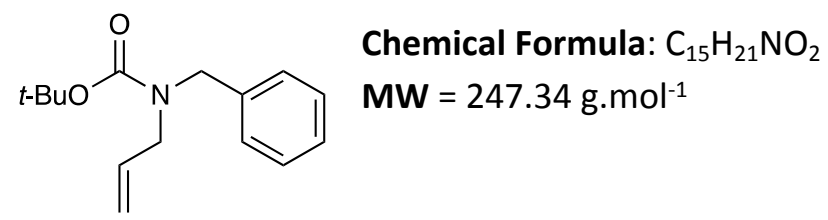

$\mathrm{NaH}$ ( $240 \mathrm{mg}, 6 \mathrm{mmol}, 1.5$ equiv) and allyl bromide $(0.52 \mathrm{~mL}, 6 \mathrm{mmol}, 1.5$ equiv) were added to a solution of $\mathrm{N}$-Boc-benzylamine $1 \mathrm{a}^{\prime}\left(829 \mathrm{mg}, 4 \mathrm{mmol}, 1\right.$ equiv) in $\mathrm{DMF}(20 \mathrm{~mL})$ at $0{ }^{\circ} \mathrm{C}$ according to General Procedure B. N-Boc, $N$-benzyl-allylamine $1 \mathrm{a}$ was isolated after purification by flash chromatography on silica gel (petroleum ether/AcOEt $=98: 2$ ) as a colorless oil $(925 \mathrm{mg}, 94 \%$, presence of two rotamers in a ratio 1:1). The spectral data were identical to those reported in the literature.

$\boldsymbol{R}_{\mathrm{f}}=0.64\left(\mathrm{SiO}_{2}\right.$, petroleum ether $\left./ \mathrm{AcOEt}=96: 4\right)$;

IR (ATR) $v=3064,2975,2928,1689,1644,1495,1452,1406,1364,1242,1164,1138,993\left(\mathrm{~cm}^{-1}\right)$;

\footnotetext{
${ }^{1}$ M. E. Duggan, J. S. Imagire Synthesis 1989, 131-132.

${ }^{2}$ G. W. Kabalkaa, N.-S. Li, R. D. Pace Synthetic Commun. 1995, 25, 2135-2143.
} 
${ }^{1} \mathrm{H}$ NMR $\left(400 \mathrm{MHz}, \mathrm{CDCl}_{3}\right) \delta$ 7.35-7.24 (m, 5H), 5.76 (br s, 1H), 5.13 (br m, 2H), 4.42 (br s, 2H), [3.85 (br s); $3.73($ br s), 2H], 1.48 (s, 9H);

${ }^{13} \mathrm{C}$ NMR $\left(101 \mathrm{MHz}, \mathrm{CDCl}_{3}\right) \delta[155.9 ; 155.6], 138.4,133.8,128.5$ (2C), [127.9; 127.4, (2C)], 127.2, [117.0; 116.4], 79.9, [49.5; 49.3], 48.7, 28.5 (3C);

MS (+EI) m/z: $247\left(\mathrm{M}^{+}, 0.23\right), 192$ (7), 191 (51), 150 (26), 147 (5), 146 (24), 132 (10), 120 (6), 118 (7), 106 (13), 104 (6), 92 (17), 91 (100), 79 (6), 77 (5), 70 (8), 65 (13), 57 (93), 56 (42), 55 (8), 51 (5).

$(+)-N$-Boc-[(R)-phenylethyl]-amine, $1 b^{\prime}{ }^{3}$

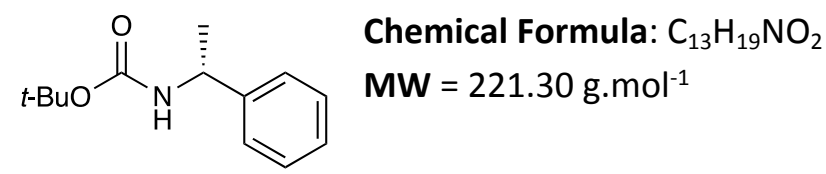

$\mathrm{Et}_{3} \mathrm{~N}$ (1.46 mL, $10.5 \mathrm{mmol}, 1.5$ equiv) and $\mathrm{Boc}_{2} \mathrm{O}(1.45 \mathrm{~g}, 6.65 \mathrm{mmol}, 0.95$ equiv) were added to a solution of $(R)$-phenylethylamine ( $0.89 \mathrm{~mL}, 7 \mathrm{mmol}, 1$ equiv) in $\mathrm{CH}_{2} \mathrm{Cl}_{2}(35 \mathrm{~mL})$ according to General Procedure $\mathrm{A}$. $\mathbf{N}$-Boc-phenylethylamine $\mathbf{1} \mathbf{b}^{\prime}$ ' was isolated after recrystallization in cold hexanes as a white solid $(1.37 \mathrm{~g}, 93 \%)$. The spectral data were identical to those reported in the literature.

m.p.: $92^{\circ} \mathrm{C}$;

$\boldsymbol{R}_{\mathrm{f}}=0.77\left(\mathrm{SiO}_{2}\right.$, petroleum ether/AcOEt = 80:20);

$[\alpha]^{20}{ }_{D}=+47\left(c 5.9, \mathrm{CHCl}_{3}\right),\left([\alpha]^{20}{ }_{D}^{\text {litt. }}=+45\left(c 0.7, \mathrm{CHCl}_{3}\right)^{4}\right) ;$

IR (ATR) $v=3378,2982,2965,1684,1514,1495,1447,1388,1363,1311,1243,1165,1075,1057$, 1028, 1008, $996\left(\mathrm{~cm}^{-1}\right)$;

${ }^{1} \mathrm{H}$ NMR $\left(400 \mathrm{MHz}, \mathrm{CDCl}_{3}\right) \delta$ 7.37-7.25 (m, 5H), $4.81(\mathrm{br} \mathrm{s}, 1 \mathrm{H}+\mathrm{NH}), 1.48-1.44(\mathrm{~m}, 12 \mathrm{H})$;

${ }^{13} \mathrm{C}$ NMR (101 MHz, $\left.\mathrm{CDCl}_{3}\right) \delta 155.2,144.2,128.7$ (2C), 127.2, $126.0(2 \mathrm{C}), 79.5,50.3,28.5$ (3C), 22.8;

MS (+EI) m/z: 107 (9), $106\left(\mathrm{M}-\mathrm{C}_{5} \mathrm{H}_{10} \mathrm{NO}_{2}+\mathrm{H}^{+*}, 100\right), 104$ (6), 92 (10), 91 (34), 79 (14), 77 (9), 65 (14).

(+)-N-Boc, $N$-[(R)-phenylethyl]-allylamine, $1 b^{5}$

Chemical Formula: $\mathrm{C}_{16} \mathrm{H}_{23} \mathrm{NO}_{2}$

\footnotetext{
${ }^{3}$ Y. Sun Park, M. L. Boys, P. Beak J. Am. Chem. Soc. 1996, 118, 3757-3758.

${ }^{4}$ S. N. Mlynarski, A. S. Karns, J. P. Morken J. Am. Chem. Soc. 2012, 134, 16449-16451.

${ }^{5}$ V. N. Wakchaure, P. S. J. Kaib, M. Leutzsch, B. List Angew. Chem. Int. Ed. 2015, 54, 11852-11856.
} 
$\mathrm{NaH}$ (136 mg, $3.39 \mathrm{mmol}, 1.5$ equiv) and allyl bromide $(0.29 \mathrm{~mL}, 3.39 \mathrm{mmol}, 1.5$ equiv) were added to a solution of $N$-Boc-[(R)-phenylethyl]-amine $1 b^{\prime}$ (500 mg, $2.26 \mathrm{mmol}, 1$ equiv) in DMF (1.17 mL) at 0 ${ }^{\circ} \mathrm{C}$ according to General Procedure B. $\mathrm{N}$-Boc, $N$-[(R)-phenylethyl]-allylamine $\mathbf{1 b}$ was isolated after purification by flash chromatography on silica gel (petroleum ether/AcOEt $=98: 2$ ) as a colorless oil (493 $\mathrm{mg}, 84 \%$, presence of two rotamers in a ratio 1:1). The spectral data were identical to those reported in the literature.

$\boldsymbol{R}_{\mathrm{f}}=0.82\left(\mathrm{SiO}_{2}\right.$, petroleum ether $/$ AcOEt $\left.=95: 5\right)$;

$[\alpha]^{25}=+97\left(c 1.70, \mathrm{CHCl}_{3}\right),\left([\alpha]^{25}{ }_{\mathrm{D}}^{\text {litt. }}=+86\left(c 1.68, \mathrm{CHCl}_{3}\right) 5\right)$

IR $($ ATR) $v=2975,2931,1684,1447,1397,1364,1321,1268,1251,1210,1169,1141,1025,986$ $\left(\mathrm{cm}^{-1}\right)$;

${ }^{1} \mathrm{H}$ NMR $\left(400 \mathrm{MHz}, \mathrm{CDCl}_{3}\right) \delta$ 7.34-7.22 (m, 5H), 5.69 (br s, 1H), 5.50 (br s, 1H), $4.99(\mathrm{~m}, 2 \mathrm{H}),[3.74(\mathrm{~m})$; $3.43(\mathrm{~m}), 2 \mathrm{H}], 1.52(\mathrm{~d}, J=7.1 \mathrm{~Hz}, 3 \mathrm{H}), 1.45(\mathrm{~s}, 9 \mathrm{H})$;

${ }^{13} \mathrm{C}$ NMR $\left(101 \mathrm{MHz}, \mathrm{CDCl}_{3}\right) \delta 155.9,142.1,136.1,128.4$ (3C), 127.2 (2C), 115.6, 79.8, 53.1, 46.2, 28.6 (3C), 17.8;

MS (+EI) m/z: 107 (9), $106\left(\mathrm{C}_{8} \mathrm{H}_{9}+\mathrm{H}^{+*}, 100\right), 104$ (6), 92 (11), 91 (35), 79 (14), 77 (9), 65 (14), 51 (5).

\section{(士)-N-Benzyl-cyclohex-2-ene-1-amine, $1 c^{\prime} 6$}

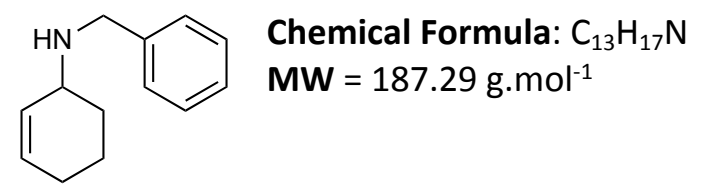

Neat Ti(OiPr) $)_{4}(2 \mathrm{~mL}, 6.76 \mathrm{mmol}, 1.69$ equiv) and benzylamine $(0.44 \mathrm{~mL}, 4 \mathrm{mmol}, 1$ equiv) were added to cyclohexenone $(0.39 \mathrm{~mL}, 4 \mathrm{mmol}, 1$ equiv) and the mixture was stirred at $\mathrm{rt}$ for $3 \mathrm{~h}$. The reaction mixture was diluted with $\mathrm{MeOH}\left(18.2 \mathrm{~mL}\right.$, dried overnight on $\left.\mathrm{MgSO}_{4}\right)$ and cooled to $0{ }^{\circ} \mathrm{C} . \mathrm{NaBH}_{4}(227$ $\mathrm{mg}, 6 \mathrm{mmol}, 1.5$ equiv) was slowly added to the media and stirred at $0{ }^{\circ} \mathrm{C}$. After $30 \mathrm{~min}$ the reaction media was quenched with a saturated aqueous $\mathrm{NaHCO}_{3}$ solution $(2 \mathrm{~mL})$ and $\mathrm{MeOH}$ was evaporated under reduced pressure. The reaction media was extracted with $\mathrm{CH}_{2} \mathrm{Cl}_{2}(3 \times 10 \mathrm{~mL})$ and the combined organic phases were dried over $\mathrm{MgSO}_{4}$, filtrated and the solvent was evaporated under reduced pressure. The crude product was purified by silica gel column chromatography (petroleum ether/AcOEt $=80: 20$ ) affording $1 \mathrm{c}^{\prime}$ as a colorless oil $(225 \mathrm{mg}, 30 \%)$. The spectral data were identical to those reported in the literature.

$\boldsymbol{R}_{\mathrm{f}}=0.21\left(\mathrm{SiO}_{2}\right.$, petroleum ether/AcOEt $\left.=80: 20\right)$

IR (ATR) $v=3300,3061,3022,2924,2856,2832,1649,1604,1494,1451,1137,1106,1075,1027$, $958\left(\mathrm{~cm}^{-1}\right)$;

${ }_{6}^{6}$ J. Pawlas, Y. Nakao, M. Kawatsura, J. F. Hartwig J. Am. Chem. Soc. 2002, 124, 3669-3679. 
${ }^{1} \mathrm{H}$ NMR $\left(400 \mathrm{MHz}, \mathrm{CDCl}_{3}\right)$ 8 7.38-7.31 (m, 4H), 7.28-7.23 (m, 1H), 5.82-5.74 (m, 2H), $3.89\left(\mathrm{~d}, \mathrm{~J}_{\mathrm{AB}}=13.0\right.$ $\mathrm{Hz}, 1 \mathrm{H}), 3.84\left(\mathrm{~d}, J_{\mathrm{AB}}=12.1 \mathrm{~Hz}, 1 \mathrm{H}\right), 3.24(\mathrm{~m}, 1 \mathrm{H}), 2.10-1.98(\mathrm{~m}, 2 \mathrm{H}), 1.95-1.88(\mathrm{~m}, 1 \mathrm{H}), 1.81-1.69(\mathrm{~m}$, $\mathrm{NH}+1 \mathrm{H}), 1.61-1.47(\mathrm{~m}, 2 \mathrm{H})$;

${ }^{13} \mathrm{C}$ NMR $\left(101 \mathrm{MHz}, \mathrm{CDCl}_{3}\right) \delta 140.6,129.8,129.2,128.5$ (2C), $128.3(2 \mathrm{C}), 127.1,52.5,51.0,29.5,25.4$, 20.3;

MS (+EI) m/z: 187 (M+•, 28), 186 (10), 172 (5), 159 (28), 158 (8), 144 (39), 108 (7), 106 (8), 96 (8), 92 (10), 91 (100), 81 (9), $80(5), 79(12), 77$ (7), 68 (8), 65 (16).

\section{( \pm )-N-Boc, $N$-benzyl-cyclohex-2-ene-1-amine, 1c}

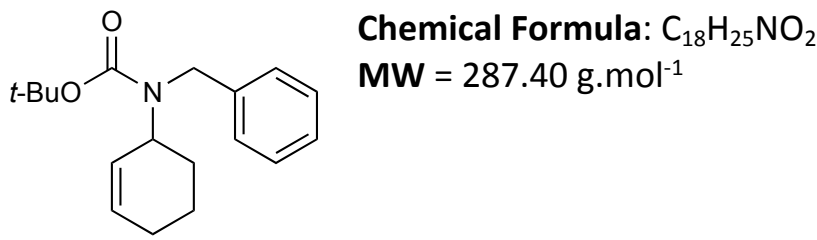

$\mathrm{Boc}_{2} \mathrm{O}$ (247 mg, $1.13 \mathrm{mmol}, 0.95$ equiv) and $\mathrm{Et}_{3} \mathrm{~N}(0.25 \mathrm{~mL}, 1.79 \mathrm{mmol}, 1.5$ equiv) were added to a solution of $\mathrm{N}$-benzyl-cyclohex-2-enyl-1-amine $1 c^{\prime}$ (223 mg, $1.19 \mathrm{mmol}, 1$ equiv) in $\mathrm{CH}_{2} \mathrm{Cl}_{2}(6 \mathrm{~mL})$ at rt for $16 \mathrm{~h}$. L-Proline (137 mg, $1.19 \mathrm{mmol}, 1$ equiv) and $\mathrm{Et}_{3} \mathrm{~N}(0.17 \mathrm{~mL}, 1.19 \mathrm{mmol}, 1$ equiv,) were added to the media and stirred at $\mathrm{rt}$ for an additional $2 \mathrm{~h}$ to remove the unreacted $\mathrm{Boc}_{2} \mathrm{O}$. A $10 \%$ aqueous solution of citric acid $(2 \mathrm{~mL})$ was added to quench the reaction. The layers were separated and the organic layer was extracted with $\mathrm{CH}_{2} \mathrm{Cl}_{2}(2 \times 10 \mathrm{~mL})$. The combined organic layers were washed with a $10 \%$ aqueous solution of citric acid $(2 \times 10 \mathrm{~mL})$ then with a saturated aqueous $\mathrm{NaHCO}_{3}$ solution $(2 \times$ $10 \mathrm{~mL})$ and brine $(2 \times 10 \mathrm{~mL})$. The organic layer was dried over $\mathrm{MgSO}_{4}$, filtrated and the solvent was evaporated under reduced pressure. The crude product was purified by silica gel column chromatography (petroleum ether/AcOEt $=95: 5$ ) affording $1 \mathrm{c}$ as a colorless oil (239 $\mathrm{mg}, 74 \%$, presence of two rotamers in a 70:30 ratio).

$\boldsymbol{R}_{\mathrm{f}}=0.36\left(\mathrm{SiO}_{2}\right.$, petroleum ether $\left./ \mathrm{AcOEt}=95: 5\right)$;

IR (ATR) $v=3027,2973,2929,2859,1686,1451,1403,1389,1364,1275,1246,1162,1115,1029$, $991\left(\mathrm{~cm}^{-1}\right)$;

${ }^{1} \mathbf{H}$ NMR $\left(400 \mathrm{MHz}, \mathrm{CDCl}_{3}\right) \delta$ 7.31-7.17 (m, 5H), $5.80(\mathrm{~m}, 1 \mathrm{H}), 5.49(\mathrm{~m}, 1 \mathrm{H}), 4.87(\mathrm{~m}, 0.7 \mathrm{H}), 4.48-4.12$ $(\mathrm{m}, 3.3 \mathrm{H}), 2.01-1.81(\mathrm{~m}, 3 \mathrm{H}), 1.75-1.65(\mathrm{~m}, 2 \mathrm{H}),[1.48(\mathrm{~s}), 1.29(\mathrm{~s}), 9 \mathrm{H}]$;

${ }^{13} \mathrm{C}$ NMR $\left(101 \mathrm{MHz}, \mathrm{CDCl}_{3}\right) \delta[156.15 ; 156.0],[140.8 ; 140.3],[131.3 ; 130.1],[128.9 ; 128.6],[128.2 ;$ $127.5(2 \mathrm{C})],[127.3 ; 127.0],[126.6 ; 126.4(2 \mathrm{C})],[80.2 ; 79.8],[54.3 ; 53.0],[47.7 ; 47.2],[31.4 ; 28.4$ (3C)], [29.8; 28.6], [26.1; 21.6], [25.7; 24.7];

HRMS (+ESI) $\mathrm{m} / z$ calcd for $\mathrm{C}_{18} \mathrm{H}_{25} \mathrm{NO}_{2} \mathrm{Na}$ [M+Na] $]^{+}: 310.1778$; found: 310.1779 . 


\section{(-)-(S)-N-Boc-2-hydroxymethylpyrrolidine, 1d' ${ }^{7}$}

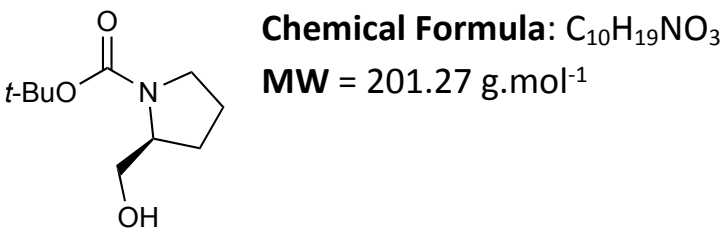

$\mathrm{BH}_{3} . \mathrm{Me}_{2} \mathrm{~S}(0.56 \mathrm{~mL}, 5.58 \mathrm{mmol}, 2$ equiv) was added to a solution of $\mathrm{N}$-Boc-L-proline (600 mg, 2.79 mmol, 1 equiv) in THF (7.7 mL) according to General Procedure C. N-Boc-2-hydroxymethylpyrrolidine 1d' was obtained without further purification as a yellowish oil $(561 \mathrm{mg}$, quantitative yield, presence of two rotamers). The spectral data were identical to those reported in the literature.

$\boldsymbol{R}_{\mathrm{f}}=0.24\left(\mathrm{SiO}_{2}\right.$, petroleum ether $/$ AcOEt $\left.=80: 20\right) ;$

$[\alpha]^{25}=-45\left(c \quad 0.4, \mathrm{CHCl}_{3}\right),\left([\alpha]^{25}{ }_{\mathrm{D}}^{\text {litt. }}=-45\left(\mathrm{c} 0.4, \mathrm{CHCl}_{3}\right)^{8}\right) ;$

IR (ATR) $v=3410,2972,2932,2876,1691,1666,1477,1454,1390,1364,1343,1250,1162,1125$, 1105, 1046, 1001, $972\left(\mathrm{~cm}^{-1}\right)$;

${ }^{1} \mathrm{H}$ NMR $\left(400 \mathrm{MHz}, \mathrm{CDCl}_{3}\right) \delta 3.92(\mathrm{~m}, 1 \mathrm{H}), 3.61\left(\mathrm{dd}, \mathrm{J}_{\mathrm{AB}}=11.1,3.7 \mathrm{~Hz}, 1 \mathrm{H}\right), 3.56\left(\mathrm{dd}, \mathrm{J}_{\mathrm{AB}}=11.1,7.3 \mathrm{~Hz}\right.$, $1 \mathrm{H}+\mathrm{OH}), 3.43\left(\mathrm{dt}_{\mathrm{App}}, J_{\mathrm{AB}}=10.8,6.8 \mathrm{~Hz}, 1 \mathrm{H}\right), 3.29\left(\mathrm{dt}_{\mathrm{App}}, J_{\mathrm{AB}}=10.8,6.8 \mathrm{~Hz}, 1 \mathrm{H}\right), 1.99(\mathrm{~m}, 1 \mathrm{H}), 1.90-1.69$ (m, 2H), $1.58(\mathrm{~m}, 1 \mathrm{H}), 1.45(\mathrm{~s}, 9 \mathrm{H})$;

${ }^{13} \mathrm{C}$ NMR $\left(101 \mathrm{MHz}, \mathrm{CDCl}_{3}\right) \delta 171.1,80.3,67.6,[60.5 ; 60.1], 47.5,28.8,28.6$ (3C), 24.0;

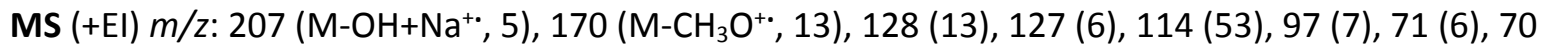
(100), 69 (12), 68 (11), 59 (14), 57 (86), 56 (61), 55 (39), 54 (5), 53 (9), 51 (6), 50 (6).

\section{(-)-(S)-N-Boc-2-formylpyrrolidine, 1d" 9}<smiles>CC(C)(C)OC(=O)N1CCC[C@H]1C=O</smiles>

Chemical Formula: $\mathrm{C}_{10} \mathrm{H}_{17} \mathrm{NO}_{3}$

$\mathrm{MW}=199.25 \mathrm{~g} \cdot \mathrm{mol}^{-1}$

DMSO (0.39 mL, 5.50 mmol, 2 equiv), $\mathrm{Et}_{3} \mathrm{~N}\left(1.53 \mathrm{~mL}, 11 \mathrm{mmol}, 4\right.$ equiv) and $\mathrm{Py} . \mathrm{SO}_{3}$ ( $875 \mathrm{mg}, 5.50 \mathrm{mmol}, 2$ equiv) were added to a solution of $\mathrm{N}$-Boc-2-hydroxymethylpyrrolidine $1 \mathrm{~d}^{\text {' (553 }}$ $\mathrm{mg}, 2.75 \mathrm{mmol}, 1$ equiv) in $\mathrm{CH}_{2} \mathrm{Cl}_{2}(9 \mathrm{~mL})$ according to General Procedure $D$. N-Boc-2formylpyrrolidine 1d" was isolated after purification by flash chromatography on silica gel (petroleum ether/AcOEt $=90: 10$ to $80: 20$ ) as a yellowish oil (303 mg, 55\%, presence of two rotamers in a ratio 65:35). The spectral data were identical to those reported in the literature.

$\boldsymbol{R}_{\mathrm{f}}=0.64\left(\mathrm{SiO}_{2}\right.$, petroleum ether $/$ AcOEt $\left.=80: 20\right) ;$

\footnotetext{
${ }^{7}$ A. G. M. Barrett, D. Pilipauska J. Org. Chem. 1990, 55, 5170-5173.

${ }^{8}$ S. Yamamotoa, H. Sugimotoa, O. Tamuraa, T. Morib, N. Matsuob, H. Ishibashi Tetrahedron 2004, 60, 8919-8927.

${ }^{9}$ I. E. Marko', A. Gautiera, J.-L. Mutonkolea, R. Dumeuniera, A. Atesa, C. J. Urchb, S. M. Brown J. Organomet. Chem. 2001, $624,344-347$.
} 
$[\alpha]^{25}=-96\left(c 0.60, \mathrm{CHCl}_{3}\right),\left([\alpha]^{25}{ }_{D}^{\text {litt. }}=-99\left(c 0.66, \mathrm{CHCl}_{3}\right)^{10}\right) ;$

IR (ATR) $v=3346,2975,2934,2879,1735,1687,1478,1455,1389,1365,1255,1160,1117,1067$, 1010, $980\left(\mathrm{~cm}^{-1}\right)$;

${ }^{1} \mathrm{H}$ NMR $\left(400 \mathrm{MHz}, \mathrm{CDCl}_{3}\right) \delta[9.53(\mathrm{~d}, J=2.8 \mathrm{~Hz}) ; 9.43(\mathrm{~d}, J=2.8 \mathrm{~Hz}), 1 \mathrm{H}],[4.18(\mathrm{~m}) ; 4.02(\mathrm{~m}), 1 \mathrm{H}]$, 3.56-3.40 (m, 2H), 2.13-1.80 (m, 4H), [1.45 (s); $1.40(\mathrm{~s}), 9 \mathrm{H}]$;

${ }^{13} \mathrm{C}$ NMR $\left(101 \mathrm{MHz}, \mathrm{CDCl}_{3}\right) \delta$ [200.8; 200.6], [154.1; 153.7], [80.8; 80.4], [65.1; 65.0], [47.0; 46.9], [28.5; 28.4, (3C)], [28.1; 26.8], [24.8; 24.1];

MS (+EI) m/z: 170 (M-CHO+*, 12), 126 (11), 114 (53), 82 (11), 71 (5), 70 (98), 57 (100), 56 (11), 55 (8).

\section{(-)-(S)-N-Boc-2-vinylpyrrolidine, $1 \mathrm{~d}^{11}$}

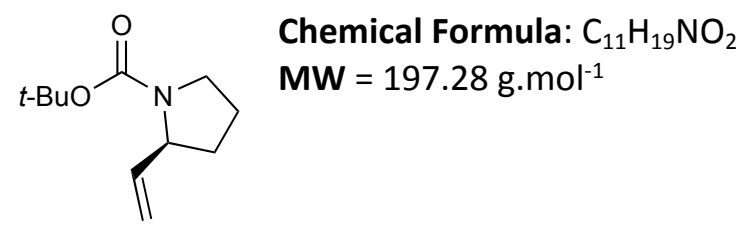

$\mathrm{N}$-Boc-2-formylpyrrolidine 1d" (100 $\mathrm{mg}, 0.50 \mathrm{mmol}, 1$ equiv) was added to a solution of methyltriphenylphosphonium bromide ( $359 \mathrm{mg}, 1.00 \mathrm{mmol}, 2$ equiv) and $n$-BuLi (0.63 mL, $c=1.59$ mol. $\mathrm{L}^{-1}, 1.00 \mathrm{mmol}, 2$ equiv) in THF $(10 \mathrm{~mL})$ according to General Procedure E. N-Boc-2Vinylpyrrolidine $1 \mathbf{d}$ was isolated after purification by flash chromatography on silica gel (petroleum ether $/$ AcOEt $=95: 5)$ as a colorless oil $(70 \mathrm{mg}, 71 \%)$. The spectral data were identical to those reported in the literature.

$\boldsymbol{R}_{\mathrm{f}}=0.42\left(\mathrm{SiO}_{2}\right.$, petroleum ether $\left./ \mathrm{AcOEt}=96: 4\right)$;

$[\alpha]^{25}=-14\left(c\right.$ 1.1, $\left.\mathrm{CHCl}_{3}\right),\left([\alpha]^{25}{ }_{\mathrm{D}}^{\mathrm{litt}}=-12\left(c \mathrm{1} .0, \mathrm{CHCl}_{3}\right)^{12}\right)$

IR (ATR) $v=3082,2973,2930,2876,1689,1643,1478,1454,1387,1363,1319,1269,1254,1164$, 1111, 1090, $987\left(\mathrm{~cm}^{-1}\right)$;

${ }^{1} \mathrm{H}$ NMR $\left(400 \mathrm{MHz}, \mathrm{CDCl}_{3}\right) \delta 5.78(\mathrm{ddd}, J=16.5,10.4,5.8 \mathrm{~Hz}, 1 \mathrm{H}), 5.06\left(\mathrm{br} \mathrm{d}_{\mathrm{App}}, J=7.0 \mathrm{~Hz}, 1 \mathrm{H}\right), 5.02(\mathrm{br}$ $\mathrm{s}, 1 \mathrm{H}), 4.27(\mathrm{~m}, 1 \mathrm{H}), 3.38(\mathrm{~m}, 2 \mathrm{H}), 1.99(\mathrm{~m}, 1 \mathrm{H}), 1.88-1.77(\mathrm{~m}, 2 \mathrm{H}), 1.69(\mathrm{~m}, 1 \mathrm{H}), 1.43(\mathrm{~s}, 9 \mathrm{H})$;

${ }^{13} \mathrm{C}$ NMR $\left(101 \mathrm{MHz}, \mathrm{CDCl}_{3}\right) \delta 154.8,139.0,113.8,79.2,59.2,46.4,31.9,28.6$ (3C), 23.2;

MS (+EI) m/z: $197\left(\mathrm{M}^{+*}, 1.20\right), 141$ (50), 124 (16), 97 (10), 96 (36), 82 (6), 81 (10), 70 (22), 69 (11), 68 (14), 57 (100), 56 (11), 55 (6).

\footnotetext{
${ }^{10}$ A. Dubey, S. R. V. Kandula, P. Kumar Syn. Commun. 2008, 38, 746-753.

${ }^{11}$ C. Serino, N. Stehle, Y. S. Park, S. Florio, P. Beak J. Org. Chem. 1999, 64, 1160-1165.

12 Y. Jagadeesh, K. K. Sridhar, R. Batchu, V. Rao Tet. Asym. 2011, 22, 1485-1489.
} 
(士)-N-Boc-2-hydroxymethylpiperidine, $1 \mathrm{e}^{\prime}{ }^{13}$

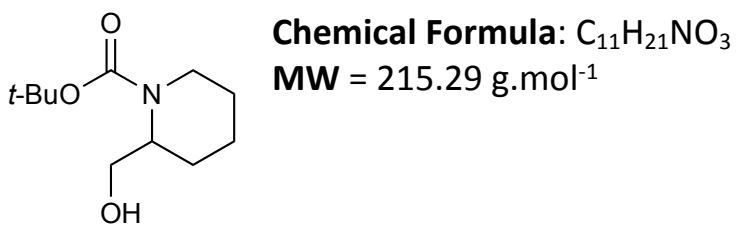

$\mathrm{BH}_{3} \cdot \mathrm{Me}_{2} \mathrm{~S}(0.57 \mathrm{~mL}, 6 \mathrm{mmol}, 2$ equiv) was added to a solution of $\mathrm{N}$-Boc-piperidine-2-carboxylic acid (633 mg, $3 \mathrm{mmol}, 1$ equiv) in THF $(8.3 \mathrm{~mL}$ ) according to General Procedure C. N-Boc-2hydroxymethylpiperidine 1e' was obtained without further purification as a yellowish oil $(517 \mathrm{mg}$, $80 \%$, presence of two rotamers in a 70:30 ratio). The spectral data were identical to those reported in the literature.

$\boldsymbol{R}_{\mathrm{f}}=0.10\left(\mathrm{SiO}_{2}\right.$, petroleum ether/AcOEt = 90:10);

IR (ATR) v = 3422, 2973, 2931, 2663, 1687, 1660, 1476, 1409, 1390, 1363, 1311, 1272, 1248, 1159, 1142, 1044, $999\left(\mathrm{~cm}^{-1}\right)$;

${ }^{1} \mathrm{H}$ NMR $\left(400 \mathrm{MHz}, \mathrm{CDCl}_{3}\right) \delta[4.62(\mathrm{~m}) ; 4.29(\mathrm{~m}), 1 \mathrm{H}], 3.93\left(\mathrm{~d}_{\mathrm{App}}, J_{\mathrm{AB}}=12.8 \mathrm{~Hz}, 1 \mathrm{H}\right), 3.82\left(\mathrm{dd}, \mathrm{J}_{\mathrm{AB}}=11.0\right.$, $9.2 \mathrm{~Hz}, 1 \mathrm{H}), 3.61\left(\mathrm{dd}, J_{\mathrm{AB}}=11.0,5.8 \mathrm{~Hz}, 1 \mathrm{H}\right), 2.86\left(\mathrm{t}_{\mathrm{App}}, J_{\mathrm{AB}}=11.9 \mathrm{~Hz}, 1 \mathrm{H}\right), 2.17(\mathrm{br} \mathrm{s}, \mathrm{OH}), 1.69-1.43(\mathrm{~m}$, $6 \mathrm{H}), 1.45(\mathrm{~s}, 9 \mathrm{H})$;

${ }^{13} \mathrm{C}$ NMR (101 MHz, CDCl 3 , major rotamer) $\delta 156.4,80.0,61.5,52.5,40.1,28.5$ (3C), 25.3 (2C), 19.7;

MS (+EI) m/z: $184\left(\mathrm{M}-\mathrm{CH}_{2} \mathrm{OH}^{+*}, 13\right), 142$ (11), 129 (7), 128 (100), 85 (6), 84 (99), 83 (10), 59 (14), 57

(85), 56 (86), 55 (43), 54 (7), 53 (9), 51 (7), 50 (7).

(士)-N-Boc-2-formylpiperidine, 1e" 13

Chemical Formula: $\mathrm{C}_{11} \mathrm{H}_{19} \mathrm{NO}_{3}$

DMSO (0.28 mL, $3.87 \mathrm{mmol}, 2$ equiv), $\mathrm{Et}_{3} \mathrm{~N}$ (1.08 mL, $7.75 \mathrm{mmol}, 4$ equiv) and Py.SO $\mathrm{SO}_{3}(617 \mathrm{mg}, 3.87$ mmol, 2 equiv) were added to a solution of $N$-Boc-2-hydroxymethylpiperidine 1e' (417 mg, 1.94 mmol, 1 equiv) in $\mathrm{CH}_{2} \mathrm{Cl}_{2}(6.5 \mathrm{~mL})$ according to General Procedure $D$. $\mathrm{N}$-Boc-2-Formylpiperidine $1 \mathrm{e}^{\mathrm{N}}$ was isolated after purification by flash chromatography on silica gel (petroleum ether/AcOEt $=90: 10$ to $80: 20$ ) as a colorless oil (194 $\mathrm{mg}, 47 \%$, presence of two rotamers in a ratio $1: 1)$. The spectral data were identical to those reported in the literature.

$\boldsymbol{R}_{\mathrm{f}}=0.45\left(\mathrm{SiO}_{2}\right.$, petroleum ether $\left./ \mathrm{AcOEt}=90: 10\right)$

IR (ATR) $v=2974,2936,2860,1733,1685,1473,1447,1391,1364,1320,1274,1248,1159,1126$, 1074, 1093, 1043, 1014, $933\left(\mathrm{~cm}^{-1}\right)$;

${ }_{13}$ M. McCrea-Hendrick, C. J. Nichols Syn. Commun. 2009, 39, 3611-3620. 
${ }^{1} \mathrm{H}$ NMR $\left(400 \mathrm{MHz}, \mathrm{CDCl}_{3}\right) \delta 9.58(\mathrm{br} \mathrm{s}, 1 \mathrm{H}), 4.55(\mathrm{~m}, 1 \mathrm{H}), 3.94(\mathrm{~m}, 1 \mathrm{H}), 2.91(\mathrm{~m}, 1 \mathrm{H}), 2.16(\mathrm{~m}, 1 \mathrm{H})$, $1.72-1.61(\mathrm{~m}, 3 \mathrm{H}), 1.46(\mathrm{~s}, 9 \mathrm{H}), 1.38-1.25(\mathrm{~m}, 2 \mathrm{H})$;

${ }^{13} \mathrm{C}$ NMR $\left(101 \mathrm{MHz}, \mathrm{CDCl}_{3}\right) \delta 201.6,[156.0 ; 155.6], 80.6,[61.7 ; 60.8],[43.2 ; 42.1], 28.4(3 \mathrm{C}), 24.8$, 23.7, 21.1;

MS (+EI) m/z: 185 (M-CHO+*, 7), 140 (8), 128 (68), 111 (7), 84 (77), 82 (15), 57 (78), 56 (100), 55 (51), 54 (12), 53 (12), 51 (9), 50 (9).

( \pm )-N-Boc-2-vinylpiperidine, $1 \mathrm{e}^{14}$<smiles>C=CC1CCCCN1C(=O)O[Na]</smiles>

$\mathrm{N}$-Boc-2-formylpiperidine 1e" (117 $\mathrm{mg}, 0.55 \mathrm{mmol}, 1$ equiv) was added to a solution of methyltriphenylphosphonium bromide (392 $\mathrm{mg}, 1.10 \mathrm{mmol}$, 2 equiv) and $n$-BuLi (0.69 mL, $c=1.59$ mol.L-1 $, 1.10 \mathrm{mmol}, 2$ equiv) in THF (11 mL) according to General Procedure E. $N$-Boc-2-vinylpiperidine 1e was isolated after purification by flash chromatography on silica gel (petroleum ether/AcOEt $=$ 95:5) as a colorless oil ( $85 \mathrm{mg}, 73 \%)$. The spectral data were identical to those reported in the literature.

$\boldsymbol{R}_{\mathrm{f}}=0.87\left(\mathrm{SiO}_{2}\right.$, petroleum ether $\left./ \mathrm{AcOEt}=90: 10\right) ;$

IR (ATR) $v=2975,2935,2861,1687,1639,1454,1402,1364,1334,1322,1271,1252,1159,1126$, 1091, 1071, 1060, 1024, $997\left(\mathrm{~cm}^{-1}\right)$;

${ }^{1} \mathrm{H}$ NMR $\left(400 \mathrm{MHz}, \mathrm{CDCl}_{3}\right) \delta 5.73(\mathrm{ddd}, J=17.4,10.6,4.1 \mathrm{~Hz}, 1 \mathrm{H}), 5.16\left(\mathrm{dt}_{\mathrm{App}}, J=10.6,1.9 \mathrm{~Hz}, 1 \mathrm{H}\right)$, $5.02\left(\mathrm{dt}_{\mathrm{App}}, J=17.4,1.5 \mathrm{~Hz}, 1 \mathrm{H}\right), 4.77(\mathrm{br} \mathrm{s}$ App, $1 \mathrm{H}), 3.93\left(\mathrm{br} \mathrm{d}_{\mathrm{App}}, J_{\mathrm{AB}}=13.4 \mathrm{~Hz}, 1 \mathrm{H}\right), 2.81\left(\mathrm{td}_{\mathrm{App}}, J_{\mathrm{AB}}=\right.$ 13.0, $2.8 \mathrm{~Hz}, 1 \mathrm{H}), 1.74-1.63(\mathrm{~m}, 2 \mathrm{H}), 1.61-1.52(\mathrm{~m}, 2 \mathrm{H}), 1.51-1.32(\mathrm{~m}, 2 \mathrm{H}), 1.44(\mathrm{~s}, 9 \mathrm{H})$;

${ }^{13} \mathrm{C}$ NMR $\left(101 \mathrm{MHz}, \mathrm{CDCl}_{3}\right) \delta 155.5,137.0,115.6,79.4,52.6,39.8,29.0,28.5$ (3C), 25.7, 19.6;

MS (+EI) m/z: 211 (M+*, 0.26), 156 (7), 155 (60), 140 (10), 138 (15), 128 (18), 126 (6), 111 (13), 110 (52), 96 (14), 84 (29), 83 (6), 82 (26), 69 (5), 68 (23), 67 (9), 57 (100), 56 (31), 55 (20), 54 (16), 53 (6).

(士)-N-Benzyl-cyclohex-2-ene-1-methanamine, 1f' ${ }^{15}$

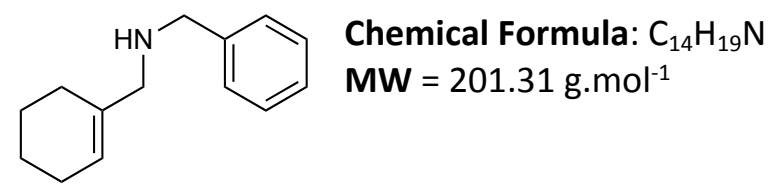

${ }^{14}$ G. A. Molander, J. A. C. Romero Tetrahedron 2005, 61, 2631-2643.

15 S. E. Steinhardt, C. D. Vanderwal J. Am. Chem. Soc. 2009, 131, 7546-7547. 
Benzylamine (0.44 mL, $4 \mathrm{mmol}, 1$ equiv) was added to a suspension of 1-cyclohexene-1carboxaldehyde ( $0.55 \mathrm{~mL}, 4.80 \mathrm{mmol}, 1.2$ equiv) and dried $\mathrm{MgSO}_{4}$ (963 mg, $8 \mathrm{mmol}, 2$ equiv) in $\mathrm{CH}_{2} \mathrm{Cl}_{2}(4 \mathrm{~mL})$ at $\mathrm{rt}$ for $1 \mathrm{~h}$. The reaction mixture was filtrated and the solvent was removed under reduced pressure. The crude product was dissolved in dry $\mathrm{MeOH}(4 \mathrm{~mL})$ and cooled to $0{ }^{\circ} \mathrm{C}$. $\mathrm{NaBH}_{4}$ (227 mg, $6 \mathrm{mmol}, 1.5$ equiv) was added portionwise then the reaction mixture was warmed up to rt and stirred overnight. The reaction was quenched with an aqueous saturated $\mathrm{NaHCO}_{3}$ solution $(2 \mathrm{~mL})$ and the solvent was evaporated under reduced pressure. The organic layer was extracted with $\mathrm{CH}_{2} \mathrm{Cl}_{2}$ $\left(3 \times 10 \mathrm{~mL}\right.$ ) and the combined organic layers were washed with an aqueous saturated $\mathrm{NaHCO}_{3}$ solution ( $2 \times 5 \mathrm{~mL}$ ). The organic layer was dried over $\mathrm{MgSO}_{4}$, filtrated and the solvent was evaporated under reduced pressure. The crude product was purified by silica gel column chromatography (petroleum ether/AcOEt $=80: 20$ ) affording $1 \mathbf{f}^{\prime}$ as a colorless oil $(764 \mathrm{mg}, 95 \%$ ). The spectral data were identical to those reported in the literature.

$\boldsymbol{R}_{\mathrm{f}}=0.44\left(\mathrm{SiO}_{2}\right.$, petroleum ether $\left./ \mathrm{AcOEt}=70: 30\right) ;$

IR (ATR) $v=3308,2921,2854,2832,1494,1451,1437,1358,1331,1158,1136,1102,1073,1027$ $\left(\mathrm{cm}^{-1}\right)$;

${ }^{1} \mathrm{H}$ NMR $\left(400 \mathrm{MHz}, \mathrm{CDCl}_{3}\right) \delta$ 7.30-7.26 (m, 4H), 7.23-7.19 (m, 1H), $5.56(\mathrm{br} \mathrm{m}, 1 \mathrm{H}), 3.71\left(\mathrm{~s}_{\text {App }}, 2 \mathrm{H}\right), 3.10$ (br s $\left.\mathrm{s}_{\text {App }}, 2 \mathrm{H}\right), 2.00-1.91(\mathrm{br} \mathrm{m}, 4 \mathrm{H}), 1.63-1.51(\mathrm{~m}, 4 \mathrm{H}+\mathrm{NH})$;

${ }^{13} \mathrm{C}$ NMR (101 MHz, $\left.\mathrm{CDCl}_{3}\right) \delta 140.7,136.2,128.5$ (2C), 128.47 (2C), 127.0, 123.0, 55.8, 53.3, 27.1, 25.2, 22.9, 22.7;

MS (+EI) m/z: 201 (M+*, 20), 200 (9), 172 (7), 133 (5), 132 (5), 120 (7), 110 (12), 106 (36), 95 (6), 93 (11), 92 (12), 91 (100), 79 (14), 77 (8), 67 (9), 65 (15), 55 (6).

\section{( \pm )-N-Boc, $N$-Benzyl-cyclohex-2-ene-1-methanamine, $1 f$}

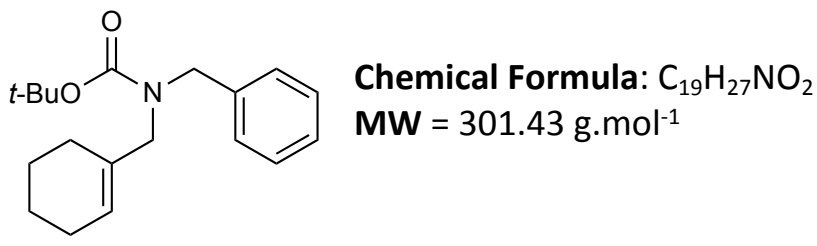

$\mathrm{Boc}_{2} \mathrm{O}$ (749 mg, $3.43 \mathrm{mmol}, 0.95$ equiv) and $\mathrm{Et}_{3} \mathrm{~N}(0.76 \mathrm{~mL}, 5.42 \mathrm{mmol}, 1.5$ equiv) were added to a solution of $\mathrm{N}$-benzyl-cyclohex-1-ene-1-methanamine $\mathbf{1 f}$ ' $726 \mathrm{mg}, 3.61 \mathrm{mmol}, 1$ equiv) in $\mathrm{CH}_{2} \mathrm{Cl}_{2}$ (18 $\mathrm{mL}$ ) at $\mathrm{rt}$ for $16 \mathrm{~h}$. L-Proline (208 mg, $1.81 \mathrm{mmol}, 0.5$ equiv) and $\mathrm{Et}_{3} \mathrm{~N}(0.25 \mathrm{~mL}, 1.81 \mathrm{mmol}, 0.5$ equiv) were added to the media and stirred at $\mathrm{rt}$ for an additional $2 \mathrm{~h}$ to remove the unreacted $\mathrm{Boc}_{2} \mathrm{O}$. A $10 \%$ aqueous solution of citric acid $(4 \mathrm{~mL})$ was added to quench the reaction. The layers were separated and the organic layer was extracted with $\mathrm{CH}_{2} \mathrm{Cl}_{2}(2 \times 20 \mathrm{~mL})$. The combined organic layers were washed with a $10 \%$ aqueous solution of citric acid $(2 \times 10 \mathrm{~mL})$ then with a saturated aqueous $\mathrm{NaHCO}_{3}$ solution $(2 \times 10 \mathrm{~mL})$ and brine $(2 \times 10 \mathrm{~mL})$. The organic layer was dried over $\mathrm{MgSO}_{4}$, filtrated and the solvent was evaporated under reduced pressure. The crude product was purified by silica gel column chromatography (petroleum ether/AcOEt = 95:5) affording $\mathbf{1}$ as a colorless oil (931 mg, 90\%, presence of two rotamers). 
$\boldsymbol{R}_{\mathbf{f}}=0.38\left(\mathrm{SiO}_{2}\right.$, petroleum ether $/$ AcOEt $\left.=95: 5\right)$;

IR (ATR) $v=2974,2925,2857,1689,1495,1412,1363,1308,1237,1162,1111,1073,1029,964$ $\left(\mathrm{cm}^{-1}\right)$;

${ }^{1} \mathrm{H}$ NMR $\left(400 \mathrm{MHz}, \mathrm{CDCl}_{3}\right) \delta$ 7.33-7.29 (m, 2H), 7.27-7.19 (m, 3H), 5.47 (br s $\left.\mathrm{s}_{\mathrm{App}}, 1 \mathrm{H}\right), 4.39\left(\mathrm{br} \mathrm{s}_{\mathrm{App}}, 1 \mathrm{H}\right)$, $4.34\left(\right.$ br s $\left.\mathrm{s}_{\text {App }}, 1 \mathrm{H}\right), 3.76\left(\mathrm{br} \mathrm{s} \mathrm{s}_{\text {App }}, 1 \mathrm{H}\right), 3.65\left(\mathrm{br} \mathrm{s}_{\text {App }}, 1 \mathrm{H}\right), 2.01$ (br $\left.\mathrm{s}_{\text {App }}, 2 \mathrm{H}\right), 1.88$ (br $\left.\mathrm{s}_{\text {App }}, 2 \mathrm{H}\right), 1.64-1.56$ $(\mathrm{m}, 4 \mathrm{H}), 1.51-1.46$ (br s, 9H);

${ }^{13} \mathrm{C}$ NMR $\left(101 \mathrm{MHz}, \mathrm{CDCl}_{3}\right) \delta[156.2 ; 156.1],[138.7 ; 138.5], 133.8,[128.6 ; 128.4,(2 \mathrm{C})],[128.1 ; 127.5$, (2C)], [127.3; 127.1], [124.4; 124.3], [80.2; 79.7], [52.3; 51.9], [49.0; 48.5], 28.5 (3C), 26.1, 25.2, 22.6, 22.5;

HRMS (+ESI) $\mathrm{m} / \mathrm{z}$ calcd for $\mathrm{C}_{19} \mathrm{H}_{27} \mathrm{NO}_{2} \mathrm{Na}[\mathrm{M}+\mathrm{Na}]^{+}:$324.1934; found: 324.1934 .

\section{Iodo-cyclization/metal-catalyzed cross-coupling sequence}

\subsection{Optimization of the iodo-cyclization step}

Table S1. Optimization of the reaction conditions for the iodo-cyclization step from the $N$-Boc, $N$-benzylallylamine 1a in presence of an iodide source.

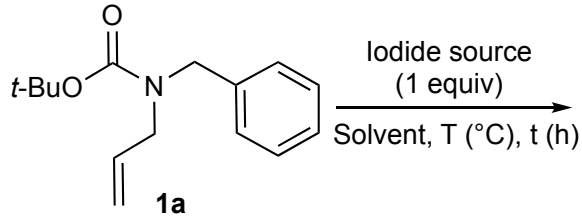<smiles>O=C1OC(CI)CN1Cc1ccccc1</smiles>

2a
Side product:<smiles>C=CCNCc1ccccc1</smiles>

2a'

\begin{tabular}{|c|c|c|c|c|c|c|c|c|}
\hline Entry & lodide source & Solvent & $\mathrm{T}\left({ }^{\circ} \mathrm{C}\right)$ & $t(h)$ & $c\left(\mathrm{~mol}^{-\mathrm{L}^{-1}}\right)$ & $2 a(\%)^{a}$ & $2 a^{\prime}(\%)^{a}$ & $\begin{array}{c}\text { Recovered } \\
\text { 1a }(\%)^{\mathrm{a}}\end{array}$ \\
\hline 1 & $I_{2}$ & $\mathrm{CH}_{2} \mathrm{Cl}_{2}$ & $\mathrm{rt}$ & 16 & 0.125 & $68^{b}$ & - & - \\
\hline 2 & $\mathrm{I}_{2}$ & THF & $\mathrm{rt}$ & 16 & 0.125 & 54 & 43 & 3 \\
\hline 3 & $\mathrm{I}_{2}$ & THF & reflux & 16 & 0.125 & 56 & 44 & - \\
\hline 4 & $\mathrm{I}_{2}$ & THF & $130^{\mathrm{b}}$ & 0.75 & 0.125 & 50 & 44 & 3 \\
\hline 5 & $\mathrm{I}_{2}$ & THF & $130^{\mathrm{b}}$ & 0.75 & 0.67 & 50 & 50 & - \\
\hline 6 & $I_{2}$ & THF & $130^{\mathrm{b}}$ & 0.16 & 0.67 & 50 & 43 & 6 \\
\hline 7 & $\mathrm{I}_{2}$ & THF & $\mathrm{rt}$ & 0.75 & 0.67 & 45 & 35 & 20 \\
\hline 8 & $\mathrm{I}_{2}$ & THF & $180^{\mathrm{b}}$ & 0.75 & 0.67 & - & - & Degradation \\
\hline 9 & $\mathrm{I}_{2}$ & 2-MeTHF & $130^{\mathrm{b}}$ & 0.75 & 0.67 & 49 & 45 & 2 \\
\hline 10 & $\mathrm{I}(\mathrm{coll})_{2} \mathrm{PF}_{6}$ & THF & $130^{\mathrm{b}}$ & 0.75 & 0.67 & 19 & 3 & 82 \\
\hline 11 & $\left(t-\mathrm{BuCO}_{2}\right)_{2} \mathrm{IPh}$ & THF & $130^{\mathrm{b}}$ & 0.75 & 0.67 & Traces & Traces & 85 \\
\hline 12 & Togni reagent & THF & $130^{\mathrm{b}}$ & 0.75 & 0.67 & - & - & 81 \\
\hline 13 & NIS & THF & $130^{\mathrm{b}}$ & 0.75 & 0.67 & 84 & 5 & 11 \\
\hline 14 & NIS & THF & $130^{\mathrm{b}}$ & 1 & 0.67 & 79 & 6 & 15 \\
\hline 15 & NIS & THF & 80 & 0.75 & 0.67 & 81 & 3 & 16 \\
\hline 16 & NIS & THF & $\mathrm{rt}$ & 0.75 & 0.67 & 8 & - & 91 \\
\hline 17 & NIS & THF & 80 & 0.50 & 0.67 & 78 & 3 & 19 \\
\hline 18 & NIS & THF & 80 & 1 & 0.67 & $77^{c}$ & 5 & 18 \\
\hline
\end{tabular}

[a] ${ }^{1} \mathrm{H}$-NMR yield determined by using 1,3-dimethoxybenzene as an internal standard. ${ }^{[b]}$ Heated under microwave irradiation. ${ }^{[c]}$ Isolated yield. 


\section{2. lodo-cyclization/metal-catalyzed cross-coupling sequence}

\section{General procedure F: One-pot iodo-cyclization/Co-, $\mathrm{Cu}$ - or Ni-catalyzed cross-coupling}

$\mathrm{N}$-lodosuccinimide (1 equiv) was added to a solution of $\mathrm{N}$-Boc-allylamine 1 (1 equiv) in THF into a sealed tube and stirred at $80^{\circ} \mathrm{C}$ for $1 \mathrm{~h}$. The reaction media was cooled to $0^{\circ} \mathrm{C}$. TMEDA (40 or $80 \mathrm{~mol}$ $\%)$ and $\mathrm{Co}(\mathrm{acac})_{3}(10 \mathrm{~mol} \%)$, $\mathrm{Cul}(5 \mathrm{~mol} \%)$ or $\mathrm{Ni}(\mathrm{acac})_{2}(2.5 \mathrm{~mol} \%)$ were added to the reaction mixture which was purged with Ar. The Grignard reagent ( 3 equiv) was added via a syringe pump $\left(1.04 \mathrm{mmol} . \mathrm{h}^{-1}\right)$. Once the addition of the Grignard reagent was completed, the reaction mixture was stirred for the indicated time. The reaction media was quenched by addition of a saturated aqueous $\mathrm{NH}_{4} \mathrm{Cl}$ solution $(1 \mathrm{~mL})$. The organic layer was extracted with AcOEt $(2 \mathrm{~mL})$ then washed with a saturated $\mathrm{NH}_{4} \mathrm{Cl}$ aqueous solution $(2 \times 3 \mathrm{~mL})$ and brine $(2 \times 3 \mathrm{~mL})$. The organic layers were combined, dried over $\mathrm{MgSO}_{4}$, filtrated and the solvent was evaporated under reduced pressure. The crude product was purified by silica gel column chromatography (petroleum ether/AcOEt $=80: 20$ to 60:40) affording the expected compound.

\subsubsection{Iodo-cyclization/cobalt-catalyzed cross-coupling sequence using arylmagnesium bromide}

Table S2. Optimization of the reaction conditions for the cross-coupling in a one-pot sequence to access $3 a$ from the $N$-Boc, $N$-benzyl-allylamine 1 a, using phenylmagnesium bromide.

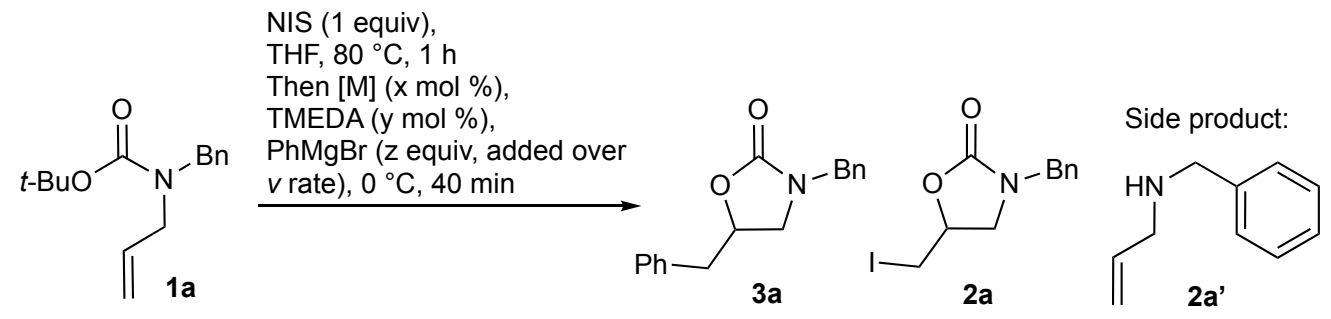

\begin{tabular}{|c|c|c|c|c|c|c|c|c|}
\hline Entry & $\begin{array}{c}{[\mathrm{M}]} \\
(\mathrm{x} \mathrm{mol} \mathrm{\% )}\end{array}$ & $\begin{array}{l}\text { TMEDA } \\
\text { (y mol \%) }\end{array}$ & $\begin{array}{l}\mathrm{PhMgBr} \\
\text { (z equiv) }\end{array}$ & 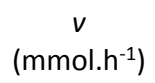 & $3 a(\%)^{a}$ & $2 a(\%)^{a}$ & $2 a^{\prime}(\%)^{a}$ & $\begin{array}{c}\text { Recovered } \\
\text { 1a (\%) }\end{array}$ \\
\hline 1 & $\mathrm{CuCl}_{2}(15)$ & None & 1.5 & 0.26 & None & 77 & 4 & 19 \\
\hline 2 & Cul (15) & None & 1.5 & 0.26 & None & 77 & 4 & 19 \\
\hline 3 & $\mathrm{FeCl}_{3}(15)$ & 100 & 1.5 & 0.26 & 6 & 64 & 8 & 22 \\
\hline 4 & $\mathrm{Fe}(\mathrm{acac})_{3}(15)$ & 100 & 1.5 & 0.26 & 8 & 59 & 12 & 20 \\
\hline 5 & $\mathrm{FeCl}_{2}(15)$ & 100 & 1.5 & 0.26 & 9 & 62 & 10 & 18 \\
\hline 6 & $\mathrm{CoCl}_{2}(15)$ & 100 & 1.5 & 0.26 & Traces & 77 & 5 & 18 \\
\hline 7 & $\mathrm{CoCl}_{2}(15)$ & 100 & 2 & 0.26 & Traces & 74 & 7 & 19 \\
\hline 8 & $\mathrm{Co}(\mathrm{acac})_{2}(15)$ & 100 & 1.5 & 0.26 & 9 & 72 & 3 & 16 \\
\hline 9 & $\mathrm{Co}(\mathrm{acac})_{3}(15)$ & 100 & 1.5 & 0.26 & 11 & 68 & 5 & 16 \\
\hline 10 & $\mathrm{Co}(\mathrm{acac})_{3}(15)$ & 100 & 2 & 0.26 & 17 & 60 & 6 & 17 \\
\hline 11 & $\mathrm{Co}(\mathrm{acac})_{3}(15)$ & 100 & 3 & 0.26 & 47 & 28 & 9 & 16 \\
\hline 12 & $\mathrm{Co}(\mathrm{acac})_{3}(15)$ & 100 & 3 & 0.52 & $74(74)^{b}$ & - & 11 & 15 \\
\hline 13 & $\mathrm{Co}(\mathrm{acac})_{3}(15)$ & 20 & 3 & 0.52 & 58 & - & 25 & 17 \\
\hline 14 & $\mathrm{Co}(\mathrm{acac})_{3}(5)$ & 20 & 3 & 0.52 & 64 & - & 21 & 15 \\
\hline 15 & $\mathrm{Co}(\mathrm{acac})_{3}(5)$ & 20 & 3 & 1.04 & 69 & - & 16 & 15 \\
\hline 16 & $\mathrm{Co}(\mathrm{acac})_{3}(5)$ & 40 & 3 & 1.04 & 65 & 6 & 15 & 14 \\
\hline 17 & $\mathrm{Co}(\mathrm{acac})_{3}(10)$ & 40 & 3 & 1.04 & $76(76)^{b}$ & - & 13 & 11 \\
\hline 18 & $\mathrm{Co}(\mathrm{acac})_{3}(15)$ & 100 & 3 & 1.04 & 62 & 11 & 11 & 16 \\
\hline 19 & $\mathrm{Co}(\mathrm{acac})_{3}(5)$ & 20 & 3 & 4.16 & 53 & 7 & 24 & 16 \\
\hline 20 & $\mathrm{Co}(\mathrm{acac})_{3}(5)$ & 40 & 3 & 4.16 & 65 & - & 16 & 19 \\
\hline
\end{tabular}

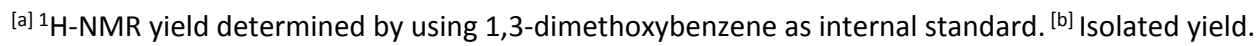




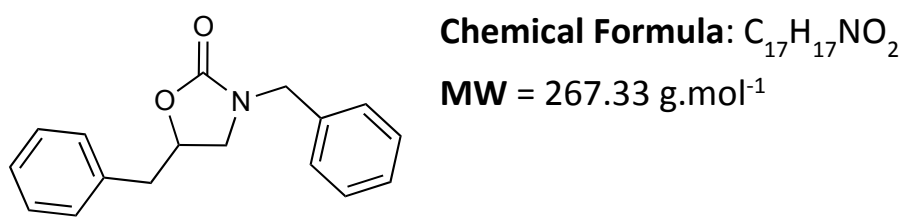

$\mathrm{N}$-lodosuccinimide (49 mg, $0.22 \mathrm{mmol}, 1$ equiv) was added to a solution of $N$-Boc, $N$-benzyl-allylamine 1a $\left(54 \mathrm{mg}, 0.22 \mathrm{mmol}, 1\right.$ equiv) in THF $\left(0.33 \mathrm{~mL}\right.$ ) and stirred at $80{ }^{\circ} \mathrm{C}$ for $1 \mathrm{~h}$. TMEDA (13 $\mu \mathrm{L}, 0.087$ $\mathrm{mmol}, 40 \mathrm{~mol} \%), \mathrm{Co}(\mathrm{acac})_{3}(8 \mathrm{mg}, 0.022 \mathrm{mmol}, 10 \mathrm{~mol} \%)$ and phenylmagnesium bromide $(0.65 \mathrm{~mL}$, $1 \mathrm{~mol} . \mathrm{L}^{-1}, 0.65 \mathrm{mmol}$ ) were added to the reaction mixture according to General Procedure $F$ and stirred for $40 \mathrm{~min}$. $\mathrm{N}$-Benzyl-5-benzyl-oxazolidin-2-one $3 \mathrm{3a}$ was isolated after purification by flash chromatography on silica gel (petroleum ether/AcOEt $=80: 20$ to $60: 40$ ) as a yellowish oil $(44 \mathrm{mg}$, $76 \%)$.

$\boldsymbol{R}_{\mathrm{f}}=0.55\left(\mathrm{SiO}_{2}\right.$, petroleum ether $\left./ \mathrm{AcOEt}=60: 40\right) ;$

IR (ATR) $v=3028,2921,1737,1603,1494,1426,1370,1348,1248,1202,1182,1096,1061,1027$, $957\left(\mathrm{~cm}^{-1}\right)$;

${ }^{1} \mathrm{H}$ NMR $\left(400 \mathrm{MHz}, \mathrm{CDCl}_{3}\right)$ 8 7.36-7.29 (m, 6H), 7.20-7.26 (m, 4H), $4.75(\mathrm{~m}, 1 \mathrm{H}), 4.38(\mathrm{~s}, 2 \mathrm{H}), 3.39\left(\mathrm{t}_{\mathrm{app}}\right.$, $\left.J_{A B}=8.9 \mathrm{~Hz}, 1 \mathrm{H}\right), 3.13\left(\mathrm{dd}, J_{\mathrm{AB}}=6.6\right.$ and $\left.8.8 \mathrm{~Hz}, 1 \mathrm{H}\right), 3.08\left(\mathrm{dd}, J_{\mathrm{AB}}=5.9\right.$ and $\left.14.0 \mathrm{~Hz}, 1 \mathrm{H}\right), 2.92\left(\mathrm{dd}, J_{\mathrm{AB}}=\right.$ 6.7 and $14.0 \mathrm{~Hz}, 1 \mathrm{H})$;

${ }^{13} \mathrm{C}$ NMR (101 MHz, CDCl ${ }_{3}$ ) $\delta 158.0,135.8,135.1,129.6$ (2C), 128.9 (2C), 128.8 (2C), $128.1(2 \mathrm{C}), 128.0$, $127.2,73.7,48.30,48.25,40.7$;

HRMS (+ESI) $m / z$ calcd for $\mathrm{C}_{17} \mathrm{H}_{18} \mathrm{NO}_{2}[\mathrm{M}+\mathrm{H}]^{+}: 268.1332$; found: 268.1333 .

\section{( \pm )-N-Benzyl-5-(p-fluorobenzyl)-oxazolidin-2-one, 3b}

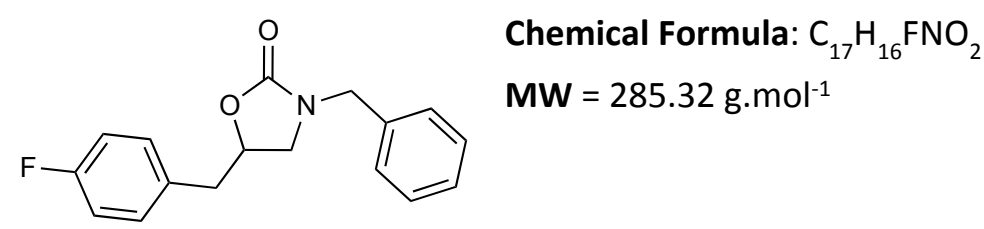

$N$-lodosuccinimide (49 mg, $0.22 \mathrm{mmol}, 1$ equiv) was added to a solution of $N$-Boc, $N$-benzyl-allylamine 1a $\left(54 \mathrm{mg}, 0.22 \mathrm{mmol}, 1\right.$ equiv) in THF $\left(0.33 \mathrm{~mL}\right.$ ) and stirred at $80^{\circ} \mathrm{C}$ for $1 \mathrm{~h}$. TMEDA (13 $\mu \mathrm{L}, 0.087$ $\mathrm{mmol}, 40 \mathrm{~mol} \%), \mathrm{Co}(\mathrm{acac})_{3}(8 \mathrm{mg}, 0.022 \mathrm{mmol}, 10 \mathrm{~mol} \%)$ and $p$-fluorophenylmagnesium bromide $\left(0.82 \mathrm{~mL}, 0.80 \mathrm{~mol} . \mathrm{L}^{-1}, 0.65 \mathrm{mmol}\right)$ were added to the reaction mixture according to General Procedure $F$ and stirred for $40 \mathrm{~min}$. N-Benzyl-5-( $p$-fluorobenzyl)-oxazolidin-2-one $\mathbf{3 b}$ was isolated after purification by flash chromatography on silica gel (petroleum ether/AcOEt $=80: 20$ to $70: 30$ ) as a white solid (40 mg, 65\%).

m.p.: $70^{\circ} \mathrm{C}$; 
$\boldsymbol{R}_{\mathrm{f}}=0.46\left(\mathrm{SiO}_{2}\right.$, petroleum ether/AcOEt $\left.=60: 40\right)$

IR (ATR) $v=3058,2955,2929,1740,1725,1605,1505,1495,1444,1427,1366,1351,1302,1269$, 1217, 1193, 1158, 1092, 1062, 1013, $959\left(\mathrm{~cm}^{-1}\right)$;

${ }^{1} \mathrm{H}$ NMR $\left(400 \mathrm{MHz}, \mathrm{CDCl}_{3}\right) \delta$ 7.34-7.26 (m, 3H), 7.16-7.11 (m, 4H), $6.97($ br t, J = 8.7 Hz, 2H), $4.70(\mathrm{~m}$, $1 \mathrm{H}), 4.38\left(\mathrm{~d}, J_{\mathrm{AB}}=15.0 \mathrm{~Hz}, 1 \mathrm{H}\right), 4.30\left(\mathrm{~d}, J_{\mathrm{AB}}=15.0 \mathrm{~Hz}, 1 \mathrm{H}\right), 3.40\left(\mathrm{t}_{\mathrm{app}}, J_{\mathrm{AB}}=8.7 \mathrm{~Hz}, 1 \mathrm{H}\right), 3.08\left(\mathrm{dd}, J_{\mathrm{AB}}=\right.$ 6.6 and $8.7 \mathrm{~Hz}, 1 \mathrm{H}), 2.97\left(\mathrm{dd}, J_{A B}=6.0\right.$ and $\left.14.3 \mathrm{~Hz}, 1 \mathrm{H}\right), 2.90\left(\mathrm{dd}, J_{\mathrm{AB}}=5.9\right.$ and $\left.14.3 \mathrm{~Hz}, 1 \mathrm{H}\right)$;

${ }^{13} \mathrm{C}$ NMR $\left(101 \mathrm{MHz}, \mathrm{CDCl}_{3}\right) \delta 161.1\left(\mathrm{~d}, J_{C-F}=245.6 \mathrm{~Hz}\right), 157.9,135.7,131.2\left(\mathrm{~d}, J_{C-F}=7.8 \mathrm{~Hz}, 2 \mathrm{C}\right), 130.8$ $\left(d, J_{C-F}=3.3 \mathrm{~Hz}\right), 128.9(2 \mathrm{C}), 128.1(2 \mathrm{C}), 128.0,115.7,115.6,73.4,48.3,48.0,39.8$;

${ }^{19} \mathrm{~F}$ NMR $\left(376 \mathrm{MHz}, \mathrm{CDCl}_{3}\right) \delta-115.56(\mathrm{~s}, 1 \mathrm{~F})$;

HRMS (+ESI) $\mathrm{m} / \mathrm{z}$ calcd for $\mathrm{C}_{17} \mathrm{H}_{16} \mathrm{FNO}_{2} \mathrm{Na}[\mathrm{M}+\mathrm{Na}]^{+}$: 308.1057; found: 308.1058 .

( \pm )-N-Benzyl-5-(p-methylbenzyl)-oxazolidin-2-one, 3c

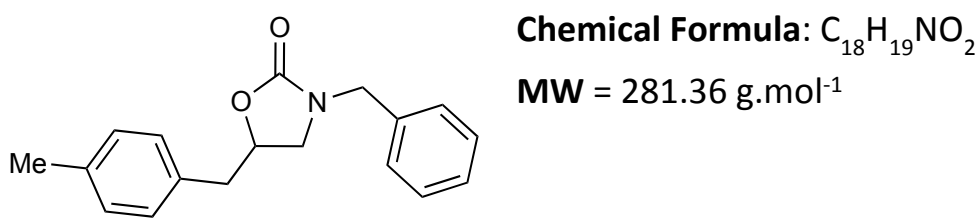

$\mathrm{N}$-lodosuccinimide (49 $\mathrm{mg}, 0.22 \mathrm{mmol}, 1$ equiv) was added to a solution of $N$-Boc, $N$-benzyl-allylamine 1a $\left(54 \mathrm{mg}, 0.22 \mathrm{mmol}, 1\right.$ equiv) in THF $(0.33 \mathrm{~mL})$ and stirred at $80{ }^{\circ} \mathrm{C}$ for $1 \mathrm{~h}$. TMEDA (13 $\mu \mathrm{L}, 0.087$ $\mathrm{mmol}, 40 \mathrm{~mol} \%), \mathrm{Co}(\mathrm{acac})_{3}(8 \mathrm{mg}, 0.022 \mathrm{mmol}, 10 \mathrm{~mol} \%)$ and $p$-tolylmagnesium bromide $(0.81 \mathrm{~mL}$, 0.81 mol. $\mathrm{L}^{-1}, 0.65 \mathrm{mmol}$ ) were added to the reaction mixture according to General Procedure $F$ and stirred for $40 \mathrm{~min}$. $\mathrm{N}$-Benzyl-5-( $p$-methylbenzyl)-oxazolidin-2-one $\mathbf{3 c}$ was isolated after purification by flash chromatography on silica gel (petroleum ether/AcOEt $=80: 20$ to 70:30) as a yellowish oil (42 $\mathrm{mg}, 68 \%)$.

$\boldsymbol{R}_{\mathrm{f}}=0.85\left(\mathrm{SiO}_{2}\right.$, petroleum ether $/$ AcOEt $\left.=60: 40\right)$

IR (ATR) $v=3026,2919,2860,1740,1690,1489,1426,1364,1347,1248,1202,1182,1091,1028$, $956\left(\mathrm{~cm}^{-1}\right)$;

${ }^{1}$ H NMR (400 MHz, $\left.\mathrm{CDCl}_{3}\right) \delta$ 7.32-7.26 (m, 3H), 7.16-7.14 (m, 2H), 7.10 (d, $\left.J=8.0 \mathrm{~Hz}, 2 \mathrm{H}\right), 7.05(\mathrm{~d}, J=$ $8.1 \mathrm{~Hz}, 2 \mathrm{H}), 4.71(\mathrm{~m}, 1 \mathrm{H}), 4.36(\mathrm{~s}, 2 \mathrm{H}), 3.36\left(\mathrm{t}_{\mathrm{app}}, J_{\mathrm{AB}}=8.6 \mathrm{~Hz}, 1 \mathrm{H}\right), 3.11\left(\mathrm{dd}, J_{\mathrm{AB}}=6.6\right.$ and $\left.8.7 \mathrm{~Hz}, 1 \mathrm{H}\right)$, $3.02\left(\mathrm{dd}, \mathrm{J}_{\mathrm{AB}}=5.7\right.$ and $\left.14.0 \mathrm{~Hz}, 1 \mathrm{H}\right), 2.86\left(\mathrm{dd}, \mathrm{J}_{\mathrm{AB}}=6.8\right.$ and $\left.14.0 \mathrm{~Hz}, 1 \mathrm{H}\right), 2.33(\mathrm{~s}, 3 \mathrm{H})$;

${ }^{13} \mathrm{C}$ NMR $\left(101 \mathrm{MHz}, \mathrm{CDCl}_{3}\right) \delta 158.1,136.8,135.8,131.9,129.5$ (2C), $129.4(2 \mathrm{C}), 128.9(2 \mathrm{C}), 128.1(2 \mathrm{C})$, $127.9,73.8,48.3,48.2,40.2,21.1$;

HRMS (+ESI) $m / z$ calcd for $\mathrm{C}_{18} \mathrm{H}_{19} \mathrm{NO}_{2} \mathrm{Na}[\mathrm{M}+\mathrm{Na}]^{+}:$304.1308; found: 304.1309 . 


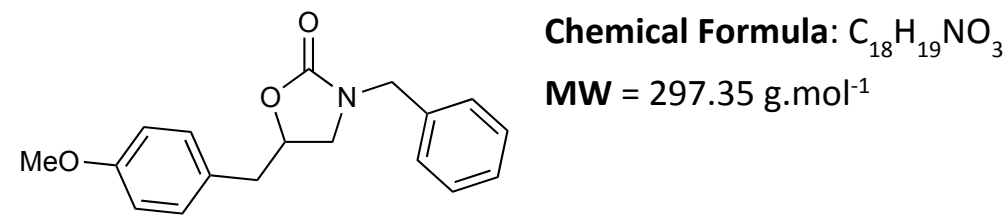

$N$-lodosuccinimide (49 mg, $0.22 \mathrm{mmol}, 1$ equiv) was added to a solution of $N$-Boc, $N$-benzyl-allylamine 1a $\left(54 \mathrm{mg}, 0.22 \mathrm{mmol}, 1\right.$ equiv) in THF $\left(0.33 \mathrm{~mL}\right.$ ) and stirred at $80{ }^{\circ} \mathrm{C}$ for $1 \mathrm{~h}$. TMEDA (13 $\mu \mathrm{L}, 0.087$ $\mathrm{mmol}, 40 \mathrm{~mol} \%), \mathrm{Co}(\mathrm{acac})_{3}(8 \mathrm{mg}, 0.022 \mathrm{mmol}, 10 \mathrm{~mol} \%)$ and $p$-methoxyphenylmagnesium bromide (1.39 mL, $\left.0.47 \mathrm{~mol} . \mathrm{L}^{-1}, 0.65 \mathrm{mmol}\right)$ were added to the reaction mixture according to General Procedure $F$ and stirred for $40 \mathrm{~min}$. N-Benzyl-5-( $p$-methoxybenzyl)-oxazolidin-2-one $\mathbf{3 d}$ was isolated after purification by flash chromatography on silica gel (petroleum ether/AcOEt $=80: 20$ to $70: 30$ ) as a white solid (40 mg, 62\%).

m.p.: $80^{\circ} \mathrm{C}$;

$\boldsymbol{R}_{\mathrm{f}}=0.61\left(\mathrm{SiO}_{2}\right.$, petroleum ether $\left./ \mathrm{AcOEt}=60: 40\right)$

IR (ATR) $v=3063,3009,2961,2928,2869,2835,1724,1609,1581,1510,1496,1490,1444,1432$, $1375,1366,1298,1266,1245,1210,1181,1114,1097,1074,1061,1027,1011,960\left(\mathrm{~cm}^{-1}\right)$;

${ }^{1} \mathrm{H}$ NMR $\left(400 \mathrm{MHz}, \mathrm{CDCl}_{3}\right) \delta$ 7.33-7.26 (m, 3H), 7.14-7.11 (m, 2H), $7.08(\mathrm{br} \mathrm{d}, J=8.7 \mathrm{~Hz}, 2 \mathrm{H}), 6.82(\mathrm{br}$ $\mathrm{d}, J=8.7 \mathrm{~Hz}, 2 \mathrm{H}), 4.69(\mathrm{~m}, 1 \mathrm{H}), 4.37\left(\mathrm{~d}, J_{\mathrm{AB}}=15.0 \mathrm{~Hz}, 1 \mathrm{H}\right), 4.32\left(\mathrm{~d}, J_{\mathrm{AB}}=15.0 \mathrm{~Hz}, 1 \mathrm{H}\right), 3.79(\mathrm{~s}, 3 \mathrm{H}), 3.36$ $\left(\mathrm{t}, J_{A B}=9.2 \mathrm{~Hz}, 1 \mathrm{H}\right), 3.10\left(\mathrm{dd}, J_{A B}=6.6\right.$ and $\left.8.7 \mathrm{~Hz}, 1 \mathrm{H}\right), 2.97\left(\mathrm{dd}, J_{A B}=5.6\right.$ and $\left.14.2 \mathrm{~Hz}, 1 \mathrm{H}\right), 2.86(\mathrm{dd}$, $J_{A B}=6.6$ and $\left.14.2 \mathrm{~Hz}, 1 \mathrm{H}\right)$;

${ }^{13} \mathrm{C}$ NMR (101 MHz, CDCl $) \delta 158.9,158.1,135.8,130.6$ (2C), 128.9 (2C), $128.0(2 \mathrm{C}), 127.95$ (2C), 127.0, 114.2, 73.8, 55.4, 48.3, 48.1, 39.7;

HRMS (+ESI) $m / z$ calcd for $\mathrm{C}_{18} \mathrm{H}_{19} \mathrm{NO}_{3} \mathrm{Na}[\mathrm{M}+\mathrm{Na}]^{+}: 320.1257$; found: 320.1257 .

( \pm )-N-Benzyl-5-(m-methoxybenzyl)-oxazolidin-2-one, 3e<smiles>COc1cccc(CC2CN(Cc3ccccc3)C(=O)O2)c1</smiles>

$N$-lodosuccinimide (49 mg, $0.22 \mathrm{mmol}, 1$ equiv) was added to a solution of $N$-Boc, $N$-benzyl-allylamine 1a (54 mg, $0.22 \mathrm{mmol}, 1$ equiv) in THF $\left(0.33 \mathrm{~mL}\right.$ ) and stirred at $80{ }^{\circ} \mathrm{C}$ for $1 \mathrm{~h}$. TMEDA (13 $\mu \mathrm{L}, 0.087$ mmol, $40 \mathrm{~mol} \%), \mathrm{Co}(\mathrm{acac})_{3}(8 \mathrm{mg}, 0.022 \mathrm{mmol}, 10 \mathrm{~mol} \%)$ and $m$-methoxyphenylmagnesium bromide $\left(0.82 \mathrm{~mL}, 0.80 \mathrm{~mol} . \mathrm{L}^{-1}, 0.65 \mathrm{mmol}\right)$ were added to the reaction mixture according to General Procedure $F$ and stirred for $40 \mathrm{~min}$. $\mathrm{N}$-Benzyl-5-( $m$-methoxybenzyl)-oxazolidin-2-one $3 \mathrm{e}$ was isolated after purification by flash chromatography on silica gel (petroleum ether/AcOEt $=80: 20$ to $70: 30$ ) as a yellowish oil (39 $\mathrm{mg}, 60 \%$ ). 
$\boldsymbol{R}_{\mathrm{f}}=0.64\left(\mathrm{SiO}_{2}\right.$, petroleum ether $\left./ \mathrm{AcOEt}=60: 40\right)$

IR (ATR) $v=3029,2922,2835,1739,1600,1583,1489,1430,1365,1347,1257,1152,1098,1036$ $950\left(\mathrm{~cm}^{-1}\right)$;

${ }^{1} \mathrm{H}$ NMR $\left(400 \mathrm{MHz}, \mathrm{CDCl}_{3}\right) \delta$ 7.34-7.26 (m, 3H), $7.05(\mathrm{~m}, 3 \mathrm{H}), 6.79-6.60(\mathrm{~m}, 3 \mathrm{H}), 4.73(\mathrm{~m}, 1 \mathrm{H}), 4.36(\mathrm{~s}$, $2 \mathrm{H}), 3.77(\mathrm{~s}, 3 \mathrm{H}), 3.37\left(\mathrm{t}_{\mathrm{App}}, J_{A B}=8.6 \mathrm{~Hz}, 1 \mathrm{H}\right), 3.11\left(\mathrm{dd}, \mathrm{J}_{\mathrm{AB}}=8.7,6.6 \mathrm{~Hz}, 1 \mathrm{H}\right), 3.03\left(\mathrm{dd}, J_{A B}=14.0,5.7\right.$ $\mathrm{Hz}, 1 \mathrm{H}), 2.87\left(\mathrm{dd}, \mathrm{J}_{\mathrm{AB}}=14.0,6.8 \mathrm{~Hz}, 1 \mathrm{H}\right)$;

${ }^{13} \mathrm{C}$ NMR $\left(101 \mathrm{MHz}, \mathrm{CDCl}_{3}\right) \delta 159.9,158.0,136.6,135.8,129.8,128.9(2 \mathrm{C}), 128.0(2 \mathrm{C}), 127.9,121.8$, $115.2,112.7,73.6,55.3,48.3,48.2,40.6$;

HRMS (+ESI) $m / z$ calcd for $\mathrm{C}_{18} \mathrm{H}_{20} \mathrm{NO}_{3}[\mathrm{M}+\mathrm{H}]^{+}:$298.1438; found: 298.1439 .

(士)-N-Benzyl-5-(o-methylbenzyl)-oxazolidin-2-one, $3 f$

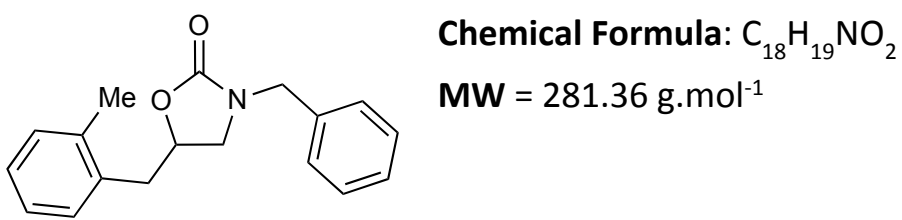

$N$-lodosuccinimide (49 mg, $0.22 \mathrm{mmol}, 1$ equiv) was added to a solution of $N$-Boc, $N$-benzyl-allylamine 1a (54 mg, $0.22 \mathrm{mmol}, 1$ equiv) in THF ( $0.33 \mathrm{~mL}$ ) and stirred at $80{ }^{\circ} \mathrm{C}$ for $1 \mathrm{~h}$. TMEDA (13 $\mu \mathrm{L}, 0.087$ $\mathrm{mmol}, 40 \mathrm{~mol} \%), \mathrm{Co}(\mathrm{acac})_{3}(8 \mathrm{mg}, 0.022 \mathrm{mmol}, 10 \mathrm{~mol} \%)$ and $o$-tolylmagnesium bromide $(0.42 \mathrm{~mL}$, $1.56 \mathrm{~mol} . \mathrm{L}^{-1}, 0.65 \mathrm{mmol}$ ) were added to the reaction mixture according to General Procedure $F$ and stirred for $40 \mathrm{~min}$. $\mathrm{N}$-Benzyl-5-(o-methylbenzyl)-oxazolidin-2-one $\mathbf{3 f}$ was isolated after purification by flash chromatography on silica gel (petroleum ether/AcOEt $=80: 20$ ) as a yellowish oil (21 mg, 34\%).

$\boldsymbol{R}_{\mathrm{f}}=0.54\left(\mathrm{SiO}_{2}\right.$, petroleum ether $\left./ \mathrm{AcOEt}=60: 40\right) ;$

IR (ATR) $v=3027,2923,2869,1739,1489,1426,1365,1347,1252,1203,1183,1096,1064,1050$, 1027, $957\left(\mathrm{~cm}^{-1}\right)$;

${ }^{1} \mathrm{H}$ NMR $\left(400 \mathrm{MHz}, \mathrm{CDCl}_{3}\right) \delta$ 7.38-7.26 (m, 3H), 7.25-7.19 (m, 2H), 7.17-7.02 (m, 4H), $4.73(\mathrm{~m}, 1 \mathrm{H})$, $4.40\left(\mathrm{~s}_{\mathrm{App}}, 2 \mathrm{H}\right), 3.39\left(\mathrm{t}_{\mathrm{App}}, J_{\mathrm{AB}}=8.5 \mathrm{~Hz}, 1 \mathrm{H}\right), 3.17-3.11(\mathrm{~m}, 2 \mathrm{H}), 2.86\left(\mathrm{dd}, J_{\mathrm{AB}}=14.3,7.3 \mathrm{~Hz}, 1 \mathrm{H}\right), 2.27(\mathrm{~s}$, $3 \mathrm{H})$;

${ }^{13} \mathrm{C}$ NMR $\left(101 \mathrm{MHz}, \mathrm{CDCl}_{3}\right) \delta 155.1,136.7,135.9,133.6,130.8,130.0,129.0$ (2C), 128.2 (2C), 128.1, $127.3,126.4,73.3,48.6,48.4,37.9,19.7$;

HRMS (+ESI) $\mathrm{m} / z$ calcd for $\mathrm{C}_{18} \mathrm{H}_{19} \mathrm{NO}_{2} \mathrm{Na}$ [M+Na] ${ }^{+}:$304.1308; found: 304.1309. 


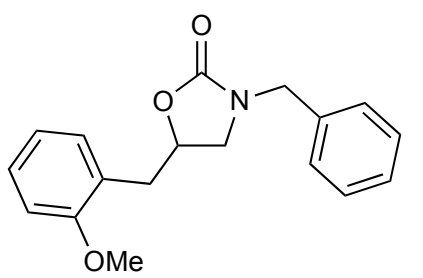

Chemical Formula: $\mathrm{C}_{18} \mathrm{H}_{19} \mathrm{NO}_{3}$

$\mathrm{MW}=297.35 \mathrm{~g} \cdot \mathrm{mol}^{-1}$

$\mathrm{N}$-lodosuccinimide (49 $\mathrm{mg}, 0.22 \mathrm{mmol}, 1$ equiv) was added to a solution of $N$-Boc, $N$-benzyl-allylamine 1a (54 mg, $0.22 \mathrm{mmol}, 1$ equiv) in THF ( $0.33 \mathrm{~mL}$ ) and stirred at $80{ }^{\circ} \mathrm{C}$ for $1 \mathrm{~h}$. TMEDA (13 $\mu \mathrm{L}, 0.087$ $\mathrm{mmol}, 40 \mathrm{~mol} \%), \mathrm{Co}(\mathrm{acac})_{3}(8 \mathrm{mg}, 0.022 \mathrm{mmol}, 10 \mathrm{~mol} \%$ ) and o-methoxyphenylmagnesium bromide $\left(0.78 \mathrm{~mL}, 0.84 \mathrm{~mol} . \mathrm{L}^{-1}, 0.65 \mathrm{mmol}\right)$ were added to the reaction mixture according to General Procedure $F$ and stirred for $40 \mathrm{~min}$. $\mathrm{N}$-Benzyl-5-(o-methoxybenzyl)-oxazolidin-2-one $\mathbf{3 g}$ was isolated after purification by flash chromatography on silica gel (petroleum ether/AcOEt $=80: 20$ to $70: 30$ ) as a yellowish oil (24 mg, 37\%).

$\boldsymbol{R}_{\mathrm{f}}=0.61\left(\mathrm{SiO}_{2}\right.$, petroleum ether $\left./ \mathrm{AcOEt}=60: 40\right)$;

IR (ATR) $v=3063,2922,2837,1741,1688,1601,1587,1493,1454,1438,1364,1242,1204,1177$, 1121, 1046, 1025, $957\left(\mathrm{~cm}^{-1}\right)$;

${ }^{1} \mathrm{H}$ NMR $\left(400 \mathrm{MHz}, \mathrm{CDCl}_{3}\right) \delta 7.35-7.19(\mathrm{~m}, 6 \mathrm{H}), 7.11(\mathrm{dd}, J=7.4,1.5 \mathrm{~Hz}, 1 \mathrm{H}), 6.88\left(\mathrm{br} \mathrm{t}_{\mathrm{App}}, J=7.4 \mathrm{~Hz}\right.$, $1 \mathrm{H}), 6.81(\mathrm{br} \mathrm{d}, J=8.2 \mathrm{~Hz}, 1 \mathrm{H}), 4.78(\mathrm{~m}, 1 \mathrm{H}), 4.40\left(\mathrm{~d}, J_{\mathrm{AB}}=15.1 \mathrm{~Hz}, 1 \mathrm{H}\right), 4.36\left(\mathrm{~d}, J_{\mathrm{AB}}=14.9 \mathrm{~Hz}, 1 \mathrm{H}\right), 3.73$ $(\mathrm{s}, 3 \mathrm{H}), 3.33\left(\mathrm{t}_{\mathrm{App}}, J_{\mathrm{AB}}=8.6 \mathrm{~Hz}, 1 \mathrm{H}\right), 3.16-3.10(\mathrm{~m}, 2 \mathrm{H}), 2.88\left(\mathrm{dd}, J_{\mathrm{AB}}=13.6,7.3 \mathrm{~Hz}, 1 \mathrm{H}\right)$;

${ }^{13} \mathrm{C}$ NMR $\left(101 \mathrm{MHz}, \mathrm{CDCl}_{3}\right) \delta 158.3,157.6,136.0,131.5,128.9$ (2C), 128.6, $128.2(2 \mathrm{C}), 127.9,123.6$, $120.8,110.5,72.9,55.3,48.5,48.3,35.3$;

HRMS (+ESI) $\mathrm{m} / \mathrm{z}$ calcd for $\mathrm{C}_{18} \mathrm{H}_{20} \mathrm{NO}_{3}[\mathrm{M}+\mathrm{H}]^{+}:$298.1438; found 298.1439 .

\section{$N$-[(R)-1-Phenylethyl]-5-benzyl-oxazolidin-2-one, 3h and 3i}

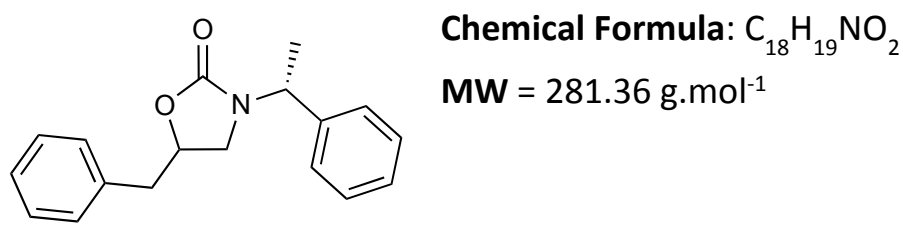

$N$-lodosuccinimide (49 mg, $0.22 \mathrm{mmol}, 1$ equiv) was added to a solution of $N$-Boc, $N$-[(R)phenylethyl] allylamine $1 \mathrm{~b}$ ( $57 \mathrm{mg}, 0.22 \mathrm{mmol}, 1$ equiv) in THF $(0.33 \mathrm{~mL})$ and stirred at $80^{\circ} \mathrm{C}$ for $1 \mathrm{~h}$. TMEDA (13 $\mu \mathrm{L}$, $0.087 \mathrm{mmol}, 40 \mathrm{~mol} \%), \mathrm{Co}(\mathrm{acac})_{3}(8 \mathrm{mg}, 0.022 \mathrm{mmol}, 10 \mathrm{~mol} \%)$ and phenylmagnesium bromide $\left(0.65 \mathrm{~mL}, 1 \mathrm{~mol} . \mathrm{L}^{-1}, 0.65 \mathrm{mmol}\right)$ were added to the reaction mixture according to General Procedure $F$ and stirred for $40 \mathrm{~min}$. The two diastereomers $\mathbf{3 h}$ and $\mathbf{3 i}$ were obtained in $62 \%$ yield with a 50:50 ratio as crude products and were separated by flash chromatography on silica gel (petroleum ether/AcOEt $=80: 20$ to $60: 40$ ). 
Reaction sequence conducted on larger scale:

$N$-lodosuccinimide (860 mg, $3.83 \mathrm{mmol}, 1$ equiv) was added to a solution of $N$-Boc, $N$-[(R)phenylethyl] allylamine $1 \mathrm{~b}\left(1 \mathrm{~g}, 3.83 \mathrm{mmol}, 1\right.$ equiv) in THF $(5.70 \mathrm{~mL})$ and stirred at $80^{\circ} \mathrm{C}$ for $1 \mathrm{~h}$. TMEDA $(0.23 \mathrm{~mL}$, $1.53 \mathrm{mmol}, 40 \mathrm{~mol} \%), \mathrm{Co}(\mathrm{acac})_{3}(136 \mathrm{mg}, 0.38 \mathrm{mmol}, 10 \mathrm{~mol} \%)$ and phenylmagnesium bromide (11.5 mL, $1 \mathrm{~mol} . \mathrm{L}^{-1}, 11.5 \mathrm{mmol}$, added through an addition rate of $11.47 \mathrm{mmol} . \mathrm{h}^{-1}$ ) were added to the reaction mixture according to General Procedure $F$ and stirred for $40 \mathrm{~min}$. The two diastereomers $\mathbf{3 h}$ and $\mathbf{3 i}$ were obtained in 55\% yield with a 60:40 ratio as crude products and were separated by flash chromatography on silica gel (petroleum ether/AcOEt $=80: 20$ to 60:40).

\section{(-)-[(R)-N-Phenylethyl]-(5S)-benzyl-oxazolidin-2-one, 3h}<smiles>C[C@H](c1ccccc1)N1C[C@H](Cc2ccccc2)OC1=O</smiles>

(-)-[(R)-N-Phenylethyl]-(5S)-benzyl-oxazolidin-2-one $\mathbf{3 h}$ was isolated as a yellowish oil (19 $\mathrm{mg}, 31 \%$ on $0.22 \mathrm{mmol}$ scale; $356 \mathrm{mg}$, 33\% on $3.83 \mathrm{mmol}$ scale).

$\boldsymbol{R}_{\mathrm{f}}=0.69\left(\mathrm{SiO}_{2}\right.$, petroleum ether $/$ AcOEt $\left.=60: 40\right) ;$

$[\alpha]^{20}{ }_{D}=-4.3\left(c \quad 1.05, \mathrm{CHCl}_{3}\right)$;

IR (ATR) $v=3060,3028,2975,2935,1734,1493,1453,1422,1365,1245,1124,1061,1021,979$ $\left(\mathrm{cm}^{-1}\right)$;

${ }^{1} \mathrm{H}$ NMR $\left(400 \mathrm{MHz}, \mathrm{CDCl}_{3}\right) \delta$ 7.36-7.06 $(\mathrm{m}, 10 \mathrm{H}), 5.18(\mathrm{q}, J=7.1 \mathrm{~Hz}, 1 \mathrm{H}), 4.71(\mathrm{~m}, 1 \mathrm{H}), 3.43\left(\mathrm{t}_{\mathrm{App}}, J_{\mathrm{AB}}=\right.$ $8.6 \mathrm{~Hz}, 1 \mathrm{H}), 2.97\left(\mathrm{dd}, J_{\mathrm{AB}}=14.0,5.7 \mathrm{~Hz}, 1 \mathrm{H}\right), 2.84\left(\mathrm{dd}, J_{\mathrm{AB}}=8.7,2.5 \mathrm{~Hz}, 1 \mathrm{H}\right), 2.78\left(\mathrm{dd}, J_{\mathrm{AB}}=14.0,6.8 \mathrm{~Hz}\right.$, $1 \mathrm{H}), 1.53(\mathrm{~d}, J=7.1 \mathrm{~Hz}, 3 \mathrm{H})$;

${ }^{13} \mathrm{C}$ NMR $\left(101 \mathrm{MHz}, \mathrm{CDCl}_{3}\right) \delta 157.5,139.5,135.1,129.6(2 \mathrm{C}), 128.8(4 \mathrm{C}), 127.8,127.2,127.0(2 \mathrm{C})$, 73.8, 51.2, 44.1, 40.6, 16.4;

HRMS (+ESI) $m / z$ calcd for $\mathrm{C}_{18} \mathrm{H}_{20} \mathrm{NO}_{2}[\mathrm{M}+\mathrm{H}]^{+}:$282.1489; found: 282.1488 .

(+)-[(R)-N-Phenylethyl]-(5R)-benzyl-oxazolidin-2-one, 3i<smiles>C[C@H](c1ccccc1)N1C[C@H](Cc2ccccc2)OC1=O</smiles>

$(+)$-[(R)-N-Phenylethyl]-(5R)-benzyl-oxazolidin-2-one $3 \mathbf{i}$ was isolated as a light green solid (19 $\mathrm{mg}, 31 \%$ on $0.22 \mathrm{mmol}$ scale; $237 \mathrm{mg}, 22 \%$ on $3.83 \mathrm{mmol}$ scale).

m.p.: $74^{\circ} \mathrm{C}$; 
$\boldsymbol{R}_{\mathrm{f}}=0.61\left(\mathrm{SiO}_{2}\right.$, petroleum ether $\left./ \mathrm{AcOEt}=60: 40\right)$

$[\alpha]^{20}{ }_{D}=+32.0\left(c 1.05, \mathrm{CHCl}_{3}\right)$

IR (ATR) v = 3060, 3028, 2974, 2934, 1734, 1603, 1488, 1453, 1423, 1365, 1247, 1122, 1061, 1021, $978\left(\mathrm{~cm}^{-1}\right)$;

${ }^{1} \mathrm{H}$ NMR $\left(400 \mathrm{MHz}, \mathrm{CDCl}_{3}\right) \delta 7.35-7.20(\mathrm{~m}, 10 \mathrm{H}), 5.13(\mathrm{q}, J=7.1 \mathrm{~Hz}, 1 \mathrm{H}), 4.65(\mathrm{~m}, 1 \mathrm{H}), 3.16\left(\mathrm{dd}, J_{\mathrm{AB}}=\right.$ 8.7, $2.0 \mathrm{~Hz}, 1 \mathrm{H}), 3.10\left(\mathrm{t}_{\mathrm{App}}, J_{\mathrm{AB}}=8.7 \mathrm{~Hz}, 1 \mathrm{H}\right), 3.04\left(\mathrm{dd}, \mathrm{J}_{\mathrm{AB}}=14.1,5.7 \mathrm{~Hz}, 1 \mathrm{H}\right), 2.94\left(\mathrm{dd}, \mathrm{J}_{\mathrm{AB}}=14.1,6.4\right.$ $\mathrm{Hz}, 1 \mathrm{H}), 1.38(\mathrm{~d}, J=7.1 \mathrm{~Hz}, 3 \mathrm{H})$;

${ }^{13} \mathrm{C}$ NMR $\left(101 \mathrm{MHz}, \mathrm{CDCl}_{3}\right) \delta 157.4,139.6,135.1,129.6(2 \mathrm{C}), 128.80$ (2C), $128.75(2 \mathrm{C}), 127.9,127.3$, $127.1(2 \mathrm{C}), 73.6,51.3,44.2,40.8,16.0$;

HRMS (+ESI) $m / z$ calcd for $\mathrm{C}_{18} \mathrm{H}_{20} \mathrm{NO}_{2}[\mathrm{M}+\mathrm{H}]^{+}:$282.1489; found: 282.1488 .

( \pm )-N-Benzyl-7-phenylhexahydrobenzo[d]oxazol-2(3H)-one, 3j

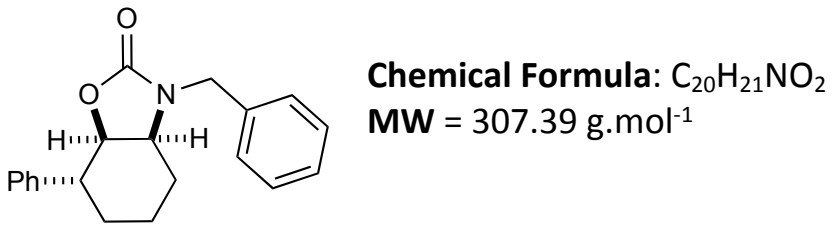

$\mathrm{N}$-lodosuccinimide ( $75 \mathrm{mg}, 0.33 \mathrm{mmol}, 1$ equiv) was added to a solution of $\mathrm{N}$-Boc, $\mathrm{N}$-benzyl-cyclohex2-ene-1-amine 1c (95 mg, $0.33 \mathrm{mmol}, 1$ equiv) in THF $(0.49 \mathrm{~mL})$ and stirred at $80{ }^{\circ} \mathrm{C}$ for $1 \mathrm{~h}$. TMEDA $(20 \mu \mathrm{L}, 0.13 \mathrm{mmol}, 40 \mathrm{~mol} \%), \mathrm{Co}(\mathrm{acac})_{3}(12 \mathrm{mg}, 0.033 \mathrm{mmol}, 10 \mathrm{~mol} \%)$ and phenylmagnesium bromide $\left(0.99 \mathrm{~mL}, 1 \mathrm{~mol} . \mathrm{L}^{-1}, 0.99 \mathrm{mmol}\right)$ were added to the reaction mixture according to General Procedure $\mathrm{F}$ and stirred for $40 \mathrm{~min}$. $\mathrm{N}$-Benzyl-7-phenylhexahydrobenzo[d]oxazol-2(3H)-one 3j was isolated as a mixture of two diastereomers in a 96:4 ratio after purification by flash chromatography on silica gel (petroleum ether/AcOEt $=80: 20$ ) as a white solid (65 $\mathrm{mg}, 64 \%$ ).

m.p.: $64^{\circ} \mathrm{C}$;

$\boldsymbol{R}_{\mathrm{f}}=0.59\left(\mathrm{SiO}_{2}\right.$, petroleum ether $/$ AcOEt $\left.=60: 40\right)$;

IR (ATR) $v=3028,2934,2862,1738,1494,1454,1432,1400,1311,1257,1200,1091,1050,1006$, $976\left(\mathrm{~cm}^{-1}\right)$;

\section{NMR Data of the major isomer:}

${ }^{1} \mathrm{H}$ NMR $\left(400 \mathrm{MHz}, \mathrm{CDCl}_{3}\right) \delta$ 7.36-7.30 (m, 7H), 7.26-7.24 (m, 3H), $4.76\left(\mathrm{~d}, \mathrm{~J}_{\mathrm{AB}}=15.3 \mathrm{~Hz}, 1 \mathrm{H}\right), 4.43$ (dd, $J=9.4,7.0 \mathrm{~Hz}, 1 \mathrm{H}$ ), $4.21\left(\mathrm{~d}, J_{\mathrm{AB}}=15.3 \mathrm{~Hz}, 1 \mathrm{H}\right), 3.77$ (ddd, $\left.J=8.0,6.9,3.9 \mathrm{~Hz}, 1 \mathrm{H}\right), 2.82$ (ddd, $J=12.0$, 9.6, $4.0 \mathrm{~Hz}, 1 \mathrm{H}), 2.08-1.84(\mathrm{~m}, 2 \mathrm{H}), 1.66-1.39(\mathrm{~m}, 4 \mathrm{H})$;

${ }^{13} \mathrm{C} \mathrm{NMR}\left(101 \mathrm{MHz}, \mathrm{CDCl}_{3}\right) \delta 159.3,142.6,136.1,128.8(2 \mathrm{C}), 128.6(2 \mathrm{C}), 128.3(2 \mathrm{C}), 127.9,127.8(2 \mathrm{C})$, 127.0, 78.7, 54.5, 46.1, 45.7, 29.2, 24.7, 19.6;

HRMS (+ESI) $\mathrm{m} / z$ calcd for $\mathrm{C}_{20} \mathrm{H}_{21} \mathrm{NO}_{2} \mathrm{Na}[\mathrm{M}+\mathrm{Na}]^{+} 330.1465$; found: 330.1465 . 


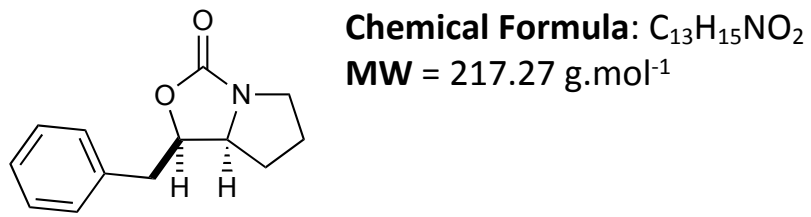

$\mathrm{N}$-lodosuccinimide (49 $\mathrm{mg}, 0.22 \mathrm{mmol}, 1$ equiv) was added to a solution of (S)-N-Boc-2vinylpyrrolidine $1 \mathrm{~d}$ (43 mg, $0.22 \mathrm{mmol}, 1$ equiv) in THF $(0.33 \mathrm{~mL})$ and stirred at $80^{\circ} \mathrm{C}$ for $1 \mathrm{~h}$. TMEDA (13 $\mu \mathrm{L}, 0.087 \mathrm{mmol}, 40 \mathrm{~mol} \%), \mathrm{Co}(\mathrm{acac})_{3}(8 \mathrm{mg}, 0.022 \mathrm{mmol}, 10 \mathrm{~mol} \%$ ) and phenylmagnesium bromide $\left(0.65 \mathrm{~mL}, 1 \mathrm{~mol} . \mathrm{L}^{-1}, 0.65 \mathrm{mmol}\right)$ were added to the reaction mixture according to General Procedure $F$ and stirred for $40 \mathrm{~min}$. 1-Benzyltetrahydro-1H,3H-pyrrolo[1,2-c]oxazol-3-one 3k was isolated as a mixture of two diastereomers in a 90:10 ratio after purification by flash chromatography on silica gel (petroleum ether/AcOEt $=80: 20$ to $70: 30$ ) as a white solid ( $24 \mathrm{mg}, 51 \%$ ).

$\boldsymbol{R}_{\mathrm{f}}=0.38\left(\mathrm{SiO}_{2}\right.$, petroleum ether $\left./ \mathrm{AcOEt}=60: 40\right)$

IR (ATR) $v=2942,2918,2896,2863,1732,1603,1495,1479,1453,1393,1370,1320,1300,1240$, $1205,1175,1139,1111,1081,1047,991\left(\mathrm{~cm}^{-1}\right)$;

${ }^{1} \mathrm{H}$ NMR $\left(400 \mathrm{MHz}, \mathrm{CDCl}_{3}\right) \delta 7.34-7.23(\mathrm{~m}, 5 \mathrm{H}),\left[4.92\left(\mathrm{q}_{\mathrm{App}}, J=7.1 \mathrm{~Hz}, 0.10 \mathrm{H}\right)\right.$ and $4.51\left(\mathrm{q}_{\mathrm{App}}, J=6.9 \mathrm{~Hz}\right.$, $0.90 \mathrm{H})$ ], [3.79-3.73 $(\mathrm{m}, 0.10 \mathrm{H}) ; 3.71-3.65(\mathrm{~m}, 0.10 \mathrm{H})$ and 3.65-3.54 $(\mathrm{m}, 0.90 \mathrm{H}$ and $0.90 \mathrm{H})], 3.20$ (dd, $\left.J_{A B}=14.0,6.4 \mathrm{~Hz}, 1 \mathrm{H}\right), 3.12(\mathrm{~m}, 1 \mathrm{H}),\left[2.95\left(\mathrm{dd}, J_{\mathrm{AB}}=14.0,7.2 \mathrm{~Hz}, 0.90 \mathrm{H}\right)\right.$ and $2.88\left(\mathrm{dd}, J_{\mathrm{AB}}=14.4,6.6\right.$ $\mathrm{Hz}, 0.10 \mathrm{H})],[2.14-2.08(\mathrm{~m}, 0.10 \mathrm{H})$ and $2.06-1.97(\mathrm{~m}, 0.90 \mathrm{H})],[1.90-1.83(\mathrm{~m}, 1.80 \mathrm{H})$ and 1.80-1.76 $(\mathrm{m}$, $0.20 \mathrm{H})],[1.61-1.55(\mathrm{~m}, 0.10 \mathrm{H})$ and $1.44-1.36(\mathrm{~m}, 0.90 \mathrm{H})]$;

${ }^{13} \mathrm{C}$ NMR (101 MHz, CDCl $) \delta$ [161.1 (major) and 160.9 (minor)], 135.7, 125.9 (2C), [129.1 (minor) and 128.9 (major) (2C)], [127.2 (major) and 127.1 (minor)], [81.1 (major) and 77.4 (minor)], [64.0 (major) and 63.4 (minor)], [45.9 (minor) and 45.6 (major)], [41.2 (major) and 36.8 (minor)], [30.9 (major) and 29.8 (minor)], [26.0 (major) and 25.2 (minor)];

HRMS (+ESI) $m / z$ calcd for $\mathrm{C}_{13} \mathrm{H}_{16} \mathrm{NO}_{2}[\mathrm{M}+\mathrm{H}]^{+}$218.1176; found: 218.1176 .

(士)-1-Benzylhexahydro-3H-oxazolo[3,4-a]pyridin-3-one, 31

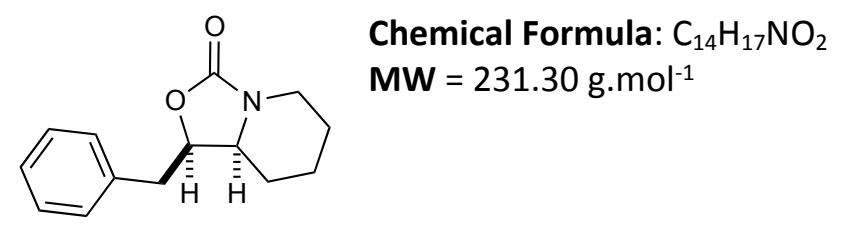

$\mathrm{N}$-lodosuccinimide ( $75 \mathrm{mg}, 0.33 \mathrm{mmol}, 1$ equiv) was added to a solution of $\mathrm{N}$-Boc-2-vinylpiperidine $1 \mathrm{e}$ (70 mg, $0.33 \mathrm{mmol}, 1$ equiv) in THF $\left(0.49 \mathrm{~mL}\right.$ ) and stirred at $80{ }^{\circ} \mathrm{C}$ for $1 \mathrm{~h}$. TMEDA ( $20 \mu \mathrm{L}, 0.13 \mathrm{mmol}$, $40 \mathrm{~mol} \%), \mathrm{Co}(\mathrm{acac})_{3}(12 \mathrm{mg}, 0.033 \mathrm{mmol}, 10 \mathrm{~mol} \%$ ) and phenylmagnesium bromide (0.99 $\mathrm{mL}, 1$ mol. $\mathrm{L}^{-1}, 0.99 \mathrm{mmol}$ ) were added to the reaction mixture according to General Procedure $F$ and stirred for $40 \mathrm{~min}$. 1-Benzylhexahydro-3H-oxazolo[3,4-a]pyridin-3-one $\mathbf{3 l}$ was isolated as a mixture of two diastereomers in a 65:35 ratio after purification by flash chromatography on silica gel (petroleum ether/AcOEt $=80: 20$ to $70: 30$ ) as a colorless oil ( $46 \mathrm{mg}, 61 \%$ ). 
$\boldsymbol{R}_{\mathrm{f}}=0.35\left(\mathrm{SiO}_{2}\right.$, petroleum ether $\left./ \mathrm{AcOEt}=60: 40\right)$

IR (ATR) $v=2936,2855,1739,1445,1419,1384,1289,1262,1240,1147,1065,1032,991\left(\mathrm{~cm}^{-1}\right)$;

${ }^{1} \mathrm{H}$ NMR $\left(400 \mathrm{MHz}, \mathrm{CDCl}_{3}\right) \delta 7.33-7.21(\mathrm{~m}, 5 \mathrm{H}),\left[4.72(\mathrm{ddd}, J=12.8,7.7,5.2 \mathrm{~Hz}, 0.35 \mathrm{H})\right.$ and $4.26\left(\mathrm{dd}_{\mathrm{App}}\right.$, $J=13.4,6.7 \mathrm{~Hz}, 0.65 \mathrm{H})],[3.89(\mathrm{~m}, 0.35 \mathrm{H})$ and $3.82(\mathrm{~m}, 0.65 \mathrm{H})],[3.65(\mathrm{ddd}, J=11.4,7.6,3.2 \mathrm{~Hz}, 0.35 \mathrm{H})$ and $3.26(\mathrm{ddd}, J=11.1,6.7,3.6 \mathrm{~Hz}, 0.65 \mathrm{H})],\left[3.14\left(\mathrm{dd}, J_{\mathrm{AB}}=14.0,6.5 \mathrm{~Hz}, 0.65 \mathrm{H}\right)\right.$ and $3.05\left(\mathrm{dd}, J_{\mathrm{AB}}=\right.$ $14.5,8.9 \mathrm{~Hz}, 0.35 \mathrm{H})], 2.90-2.80(\mathrm{~m}, 1.35 \mathrm{H}), 2.74\left(\mathrm{td}_{\mathrm{App}}, J_{\mathrm{AB}}=12.7,3.5 \mathrm{~Hz}, 0.65 \mathrm{H}\right),[1.96(\mathrm{~m}, 0.35 \mathrm{H})$ and $1.80(\mathrm{~m}, 0.65 \mathrm{H})], 1.64(\mathrm{~m}, 1 \mathrm{H}), 1.48-1.11(\mathrm{~m}, 4 \mathrm{H})$;

${ }^{13} \mathrm{C}$ NMR (101 MHz, $\left.\mathrm{CDCl}_{3}\right) \delta$ [157.1 (minor) and 156.5 (major)], [136.8 (minor) and 135.5 (major)], [129.5 (major) and 129.2 (minor) (2C)], [128.8 (major) and 128.7 (minor) (2C)], [127.1 (major) and 126.9 (minor)], [80.9 (major) and 77.3 (minor)], [59.3 (major) and 58.0 (minor)], [42.1 (minor) and 41.3 (major)], [40.1 (major) and 35.6 (minor)], [30.5 (major) and 25.3 (minor)], [24.32 (minor) and 24.30 (major)], [23.1 (minor) and 22.7 (major)];

HRMS (+ESI) $m / z$ calcd for $\mathrm{C}_{14} \mathrm{H}_{18} \mathrm{NO}_{2}[\mathrm{M}+\mathrm{H}]^{+} ; 232.1332$ found: 232.1333.

(士)-N-Benzyl-6-phenyl-1-oxa-3-azaspiro[4.5]decan-2-one, 3m<smiles>O=C1O[C@]2(CCCCC2c2ccccc2)CN1Cc1ccccc1</smiles>

$\mathrm{N}$-lodosuccinimide ( $75 \mathrm{mg}, 0.33 \mathrm{mmol}, 1$ equiv) was added to a solution of $\mathrm{N}$-Boc, $\mathrm{N}$-benzyl-cyclohex2-ene-1-methanamine 1f (100 mg, $0.33 \mathrm{mmol}, 1$ equiv) in THF $(0.49 \mathrm{~mL})$ and stirred at $80^{\circ} \mathrm{C}$ for $1 \mathrm{~h}$. TMEDA (20 $\mu \mathrm{L}, 0.13 \mathrm{mmol}, 40 \mathrm{~mol} \%), C o(\mathrm{acac})_{3}(12 \mathrm{mg}, 0.033 \mathrm{mmol}, 10 \mathrm{~mol} \%$ ) and phenylmagnesium bromide $\left(0.99 \mathrm{~mL}, 1 \mathrm{~mol} . \mathrm{L}^{-1}, 0.99 \mathrm{mmol}\right)$ were added to the reaction mixture according to General Procedure $F$ and stirred for 40 min. N-Benzyl-6-phenyl-1-oxa-3azaspiro[4.5]decan-2-one $\mathbf{3 m}$ was isolated as a mixture of two diastereomers in a 95:5 ratio after purification by flash chromatography on silica gel (petroleum ether/AcOEt $=80: 20$ ) as a white solid (12 mg, 11\%).

m.p.: $186^{\circ} \mathrm{C}$;

$\boldsymbol{R}_{\mathrm{f}}=0.64\left(\mathrm{SiO}_{2}\right.$, petroleum ether $\left./ \mathrm{AcOEt}=60: 40\right) ;$

IR (ATR) $v=2931,2854,1731,1493,1447,1439,1428,1292,1277,1210,1112,1077,1053,980$ $\left(\mathrm{cm}^{-1}\right)$;

\section{NMR Data of the major isomer:}

${ }^{1} \mathrm{H}$ NMR $\left(400 \mathrm{MHz}, \mathrm{CDCl}_{3}\right) \delta$ 7.31-7.28 (m, 3H), 7.25-7.23 (m, 2H), 7.22-7.17 (m, 3H), $6.70(\mathrm{br} \mathrm{d}, J=8.0$ $\mathrm{Hz}, 2 \mathrm{H}), 4.18\left(\mathrm{~d}, J_{\mathrm{AB}}=15.3 \mathrm{~Hz}, 1 \mathrm{H}\right), 3.94\left(\mathrm{~d}, J_{\mathrm{AB}}=15.3 \mathrm{~Hz}, 1 \mathrm{H}\right), 3.20\left(\mathrm{~d}, J_{\mathrm{AB}}=8.6 \mathrm{~Hz}, 1 \mathrm{H}\right), 3.02(\mathrm{dd}, J=$ 13.0, 3.4 Hz, $1 \mathrm{H}), 2.98\left(\mathrm{~d}, \mathrm{~J}_{\mathrm{AB}}=8.6 \mathrm{~Hz}, 1 \mathrm{H}\right), 2.05-1.82(\mathrm{~m}, 5 \mathrm{H}), 1.63-1.53(\mathrm{~m}, 1 \mathrm{H}), 1.46-1.23(\mathrm{~m}, 2 \mathrm{H})$; 
${ }^{13} \mathrm{C}$ NMR (101 MHz, CDCl $) \delta 157.2,138.7,135.5,129.0$ (2C), 128.8 (2C), $128.4(2 \mathrm{C}), 127.55$ (2C), $127.5,127.3,81.3,49.3,48.9,47.9,38.7,27.9,25.3,23.2$;

HRMS (+ESI) $m / z$ calcd for $\mathrm{C}_{21} \mathrm{H}_{23} \mathrm{NO}_{2} \mathrm{Na}[\mathrm{M}+\mathrm{Na}]^{+}: 344.1621$; found: 344.1621 .

$(+)-(R)-1-[N-(R)-$ Phenylethylamino]-propan-2-ol, 4

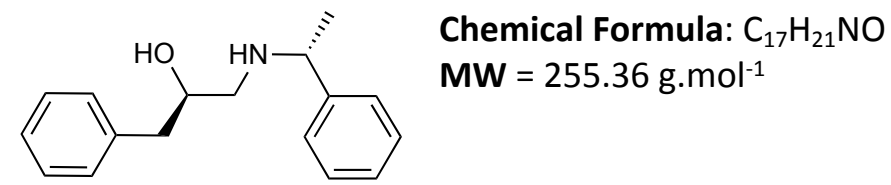

A solution of $\mathrm{NaOH}$ in EtOH ( $96 \mu \mathrm{L}, 50 \% \mathrm{~m} / \mathrm{m}, 1.20 \mathrm{mmol}, 7.5$ equiv) was added to a solution of $\mathrm{N}$-[(R)-Phenylethyl]-(5R)-benzyl-oxazolidin-2-one $3 \mathbf{i}(45 \mathrm{mg}, 0.16 \mathrm{mmol}, 1$ equiv) in EtOH (0.43 $\mathrm{mL})$ and heated to $80^{\circ} \mathrm{C}$ for $16 \mathrm{~h}$. The reaction mixture was diluted in $\mathrm{CHCl}_{3}(3 \mathrm{~mL})$ and washed with $\mathrm{H}_{2} \mathrm{O}$ $(2 \times 3 \mathrm{~mL})$. The organic layer was dried over $\mathrm{MgSO}_{4}$, filtrated and the solvent was evaporated under reduced pressure. The crude product was purified by flash chromatography on silica gel (petroleum ether/AcOEt $=70: 30$ to 0:100) affording $4(31 \mathrm{mg}, 76 \%)$ as a yellowish gummy solid.

$\boldsymbol{R}_{\mathrm{f}}=0.08\left(\mathrm{SiO}_{2}\right.$, petroleum ether $\left./ \mathrm{AcOEt}=60: 40\right) ;$

$[\alpha]_{D}^{25}=+15.8\left(c\right.$ 1.04, $\left.\mathrm{CHCl}_{3}\right)$

IR (ATR) $v=3334,3253,3020,2980,2847,2648,1494,1451,1351,1111,1100,1084,1050,1023$, $909\left(\mathrm{~cm}^{-1}\right)$;

${ }^{1} \mathrm{H}$ NMR $\left(400 \mathrm{MHz}, \mathrm{CDCl}_{3}\right) \delta$ 7.38-7.19 (m, 10H), 3.84-3.76 (m, 2H), $2.72(\mathrm{~d}, J=6.4 \mathrm{~Hz}, 2 \mathrm{H}), 2.65$ (dd, $\left.J_{A B}=12.0,3.2 \mathrm{~Hz}, 1 \mathrm{H}\right), 2.60(\mathrm{br} \mathrm{s}, \mathrm{NH}$ and $\mathrm{OH}), 2.52\left(\mathrm{dd}, J_{\mathrm{AB}}=12.0,8.8 \mathrm{~Hz}, 1 \mathrm{H}\right), 1.40(\mathrm{~d}, J=6.6 \mathrm{~Hz}, 3 \mathrm{H}$ );

${ }^{13} \mathrm{C}$ NMR (101 MHz, CDCl ${ }_{3}$ ) $\delta 145.0,138.4,129.5$ (2C), 128.7 (2C), 128.6 (2C), 127.3, 126.7 (2C), 126.5, 71.2, 58.8, 53.0, 41.7, 24.0;

HRMS (+ESI) $m / z$ calcd for $\mathrm{C}_{17} \mathrm{H}_{22} \mathrm{NO}[\mathrm{M}+\mathrm{H}]^{+}: 256.1696$; found: 256.1694 . 


\subsection{2. lodo-cyclization/copper-catalyzed cross-coupling sequence using cyclopropyl- magnesium bromide}

Table S3. Optimization of the reaction conditions for the cross-coupling in a one-pot sequence to access $3 \mathrm{n}$ from the $N$-Boc, $N$-benzyl-allylamine 1 a, using cyclopropylmagnesium bromide.

NIS (1 equiv),

$\mathrm{THF}, 80^{\circ} \mathrm{C}, 1 \mathrm{~h}$

Then $[\mathrm{M}](\mathrm{x} \mathrm{mol} \%)$,

Ligand (y mol \%),
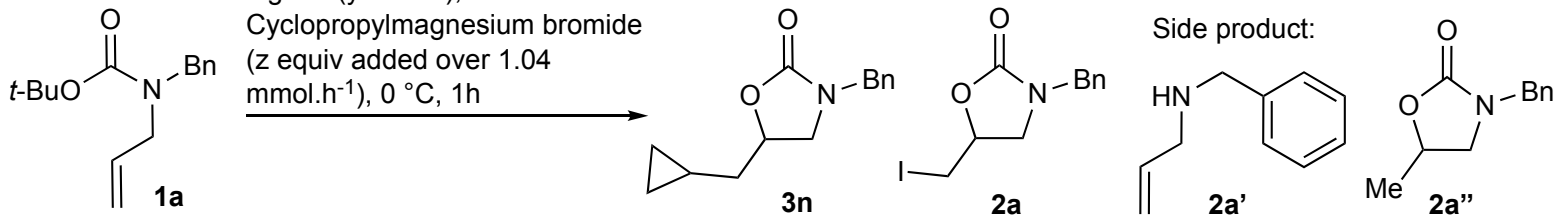

\begin{tabular}{|c|c|c|c|c|c|c|c|c|}
\hline Entry & $\begin{array}{c}{[\mathrm{M}]} \\
(\mathrm{x} \mathrm{mol} \mathrm{\% )}\end{array}$ & $\begin{array}{c}\text { Ligand } \\
\text { (y mol \%) }\end{array}$ & $\begin{array}{c}\text { Cyclopropyl- } \\
\text { magnesium } \\
\text { bromide (z equiv) }\end{array}$ & $3 n(\%)^{a}$ & $2 a(\%)^{a}$ & $2 a^{\prime}(\%)^{a}$ & $2 a^{\prime \prime}(\%)^{a}$ & $\begin{array}{c}\text { Recovered } \\
\text { 1a (\%) }\end{array}$ \\
\hline 1 & $\mathrm{Co}(\mathrm{acac})_{3}(10)$ & TMEDA (40) & 3 & $6^{b}$ & - & 51 & 12 & 28 \\
\hline 2 & $\mathrm{CoCl}_{2}(15)$ & TMEDA (100) & 1.5 & 8 & - & 58 & 4 & 30 \\
\hline 3 & $\mathrm{Co}(\mathrm{acac})_{2}(3.5)$ & TMEDA (100) & 1.2 & 8 & - & 64 & 5 & 23 \\
\hline 4 & $\mathrm{FeCl}_{2}(10)$ & TMEDA (100) & 1.2 & - & 70 & 8 & - & 22 \\
\hline 5 & $\mathrm{FeCl}_{3}(10)$ & TMEDA (100) & 1.2 & - & 65 & 10 & 5 & 20 \\
\hline 6 & $\mathrm{Fe}(\mathrm{acac})_{3}(10)$ & TMEDA (100) & 1.2 & - & 69 & 12 & - & 19 \\
\hline 7 & $\mathrm{Li}_{2} \mathrm{CuCl}_{4}(10)$ & None & 1.2 & - & 75 & 4 & - & 21 \\
\hline 8 & $\mathrm{CuCl}_{2}(10)$ & None & 1.2 & Traces & 71 & 8 & - & 20 \\
\hline 9 & $\mathrm{CuCl}(10)$ & None & 1.2 & 31 & 41 & 8 & - & 20 \\
\hline 10 & $\mathrm{CuBr}(10)$ & None & 1.2 & 34 & 43 & 5 & - & 18 \\
\hline 11 & Cul (10) & None & 1.2 & 31 & 47 & 3 & - & 19 \\
\hline 12 & Cul (10) & None & 2 & 36 & 33 & 11 & - & 20 \\
\hline 13 & Cul (10) & None & 3 & 53 & 11 & 15 & - & 21 \\
\hline 14 & Cul (10) & TMEDA (20) & 3 & 54 & 8 & 15 & - & 23 \\
\hline 15 & Cul (10) & TMEDA (40) & 3 & 64 & - & 9 & - & 27 \\
\hline 16 & Cul (15) & TMEDA (60) & 3 & 62 & - & 19 & - & 19 \\
\hline 17 & Cul (5) & TMEDA (20) & 3 & 58 & - & 16 & - & 26 \\
\hline 18 & Cul (5) & TMEDA (40) & 3 & $66^{b}$ & - & 9 & - & 24 \\
\hline 19 & Cul (5) & TMEDA (60) & 3 & 65 & - & 12 & - & 24 \\
\hline 20 & Cul (2.5) & TMEDA (20) & 3 & 48 & 12 & 17 & - & 23 \\
\hline 21 & Cul (2.5) & TMEDA (40) & 3 & 64 & - & 11 & - & 25 \\
\hline 22 & Cul (2.5) & TMEDA (60) & 3 & 60 & - & 14 & - & 26 \\
\hline 23 & $\mathrm{CuBr}(5)$ & TMEDA (20) & 3 & 55 & - & 19 & - & 26 \\
\hline 24 & $\mathrm{CuBr}(5)$ & TMEDA (40) & 3 & 64 & - & 10 & - & 25 \\
\hline 25 & Cul (5) & TMCD (20) & 3 & 54 & - & 18 & - & 28 \\
\hline 26 & Cul (5) & $\begin{array}{c}\text { 2,2-Bipyridine } \\
(20)\end{array}$ & 3 & 61 & - & 15 & - & 24 \\
\hline 27 & $\mathrm{CuBr}(5)$ & $\begin{array}{l}\text { 2,2-Bipyridine } \\
(20)\end{array}$ & 3 & 62 & - & 13 & - & 25 \\
\hline 28 & $\mathrm{CuBr}(10)$ & $\begin{array}{c}\text { 2,2-Bipyridine } \\
(40)\end{array}$ & 3 & 66 & - & 9 & - & 25 \\
\hline
\end{tabular}

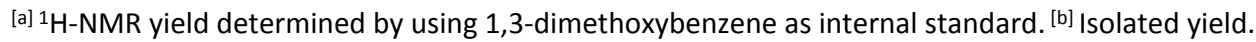


( \pm )-N-Benzyl-5-cyclopropylmethyl-oxazolidin-2-one, 3n

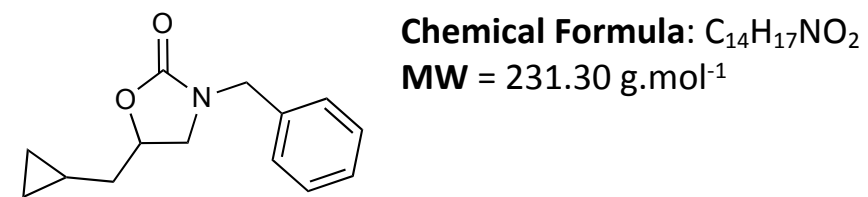

$\mathrm{N}$-lodosuccinimide (49 mg, $0.22 \mathrm{mmol}, 1$ equiv) was added to a solution of $\mathrm{N}$-Boc, $\mathrm{N}$-benzyl-allylamine 1a $\left(54 \mathrm{mg}, 0.22 \mathrm{mmol}, 1\right.$ equiv) in THF $\left(0.33 \mathrm{~mL}\right.$ ) and stirred at $80{ }^{\circ} \mathrm{C}$ for $1 \mathrm{~h}$. TMEDA (13 $\mu \mathrm{L}, 0.087$ mmol, $40 \mathrm{~mol} \%)$, Cul (1 mg, $0.011 \mathrm{mmol}, 5 \mathrm{~mol} \%)$ and cyclopropylmagnesium bromide $(0.80 \mathrm{~mL}$, $0.82 \mathrm{~mol}^{-L^{-1}}, 0.65 \mathrm{mmol}$ ) were added to the reaction mixture according to General Procedure $F$ and stirred for $1 \mathrm{~h}$. $\mathrm{N}$-Benzyl-5-cyclopropylmethyl-oxazolidin-2-one $3 \mathrm{n}$ was isolated after purification by flash chromatography on silica gel (petroleum ether/AcOEt $=80: 20$ ) as a yellowish oil (33 mg, 66\%).

$\boldsymbol{R}_{\mathrm{f}}=0.65\left(\mathrm{SiO}_{2}\right.$, petroleum ether $\left./ \mathrm{AcOEt}=60: 40\right) ;$

IR (ATR) $v=3077,3001,2918,1735,1491,1425,1364,1344,1247,1202,1183,1090,1062,1020$, $958\left(\mathrm{~cm}^{-1}\right)$;

${ }^{1} \mathrm{H}$ NMR $\left(400 \mathrm{MHz}, \mathrm{CDCl}_{3}\right) \delta 7.37-7.26(\mathrm{~m}, 5 \mathrm{H}), 4.56(\mathrm{~m}, 1 \mathrm{H}), 4.45\left(\mathrm{~d}, \mathrm{~J}_{\mathrm{AB}}=14.9 \mathrm{~Hz}, 1 \mathrm{H}\right), 4.39\left(\mathrm{~d}, \mathrm{~J}_{\mathrm{AB}}=\right.$ $14.9 \mathrm{~Hz}, 1 \mathrm{H}), 3.49\left(\mathrm{t}_{\mathrm{App}}, J_{\mathrm{AB}}=8.5 \mathrm{~Hz}, 1 \mathrm{H}\right), 3.10\left(\mathrm{dd}, J_{\mathrm{AB}}=8.6,7.1 \mathrm{~Hz}, 1 \mathrm{H}\right), 1.71\left(\mathrm{ddd}, \mathrm{J}_{\mathrm{AB}}=14.4,8.0,6.6\right.$ $\mathrm{Hz}, 1 \mathrm{H}), 1.41\left(\mathrm{ddd}, \mathrm{J}_{\mathrm{AB}}=16.0,8.0,6.1 \mathrm{~Hz}, 1 \mathrm{H}\right), 0.71(\mathrm{~m}, 1 \mathrm{H}), 0.46(\mathrm{~m}, 2 \mathrm{H}), 0.12-0.03(\mathrm{~m}, 2 \mathrm{H})$;

${ }^{13} \mathrm{C}$ NMR $\left(101 \mathrm{MHz}, \mathrm{CDCl}_{3}\right) \delta 158.4,136.1,129.0$ (2C), 128.3 (2C), 128.1, 74.1, 49.2, 48.5, 39.7, 6.4, 4.5, 4.3;

HRMS (+ESI) $\mathrm{m} / \mathrm{z}$ calcd for $\mathrm{C}_{14} \mathrm{H}_{18} \mathrm{NO}_{2}[\mathrm{M}+\mathrm{H}]^{+}:$232.1332; found: 232.1333 . 


\section{Preparation of the crystals of $3 j$ and 4 and $X$-Ray crystallographic data}

\section{Sample preparation}

\section{( \pm )-N-Benzyl-7-phenylhexahydrobenzo[d]oxazol-2(3H)-one, 3j}

In a little vial, about $30 \mathrm{mg}$ of pure $\mathbf{3} \mathbf{j}$ was solubilized in a minimum volume of ethyl acetate (about 1 $\mathrm{mL}$ ). A cap was added to close the vial and allow a slow evaporation of the solvent. After few days at room temperature, crystals grew up and were analyzed by X-ray diffraction.

\section{$(+)-(R)-1-[N-(R)-P h e n y l e t h y l a m i n o]-p r o p a n-2-o l, ~ 4$}

In a NMR tube, about $10 \mathrm{mg}$ of pure 4 was solubilized in deutarated chloroform (about $0.5 \mathrm{~mL}$ ). A cap was added to close the tube and allow a slow evaporation of the solvent. After few days at room temperature, crystals grew up and were analyzed by X-ray diffraction.

\section{X-Ray crystal structure determination}

Single crystals were selected, mounted and transferred into a cold nitrogen gas stream. Intensity data was collected with a Bruker Kappa-APEX2 system using micro-source $\mathrm{Cu}$-Ka radiation. Unit-cell parameters determination, data collection strategy, integration and absorption correction were carried out with the Bruker APEX2 suite of programs. The structures were solved with SHELXT and refined anisotropically by full-matrix least-squares methods with SHELXL using the WinGX suite. Absolute structure of $\mathbf{3 j}$ and $\mathbf{4}$ were determined by anomalous scattering effects analysis and chemical absolute configuration were then deduced. The structures were deposited at the Cambridge Crystallographic Data Centre with numbers 1991426 and 1991427, respectively, and can be obtained free of charge via www.ccdc.cam.ac.uk. 


\section{5. ${ }^{1} \mathrm{H},{ }^{19} \mathrm{~F}$ and ${ }^{13} \mathrm{C}$ NMR spectra}

N-Boc-benzylamine, 1a'

${ }^{1} \mathrm{H}$ NMR (400 MHz, $\mathrm{CDCl}_{3}$ )

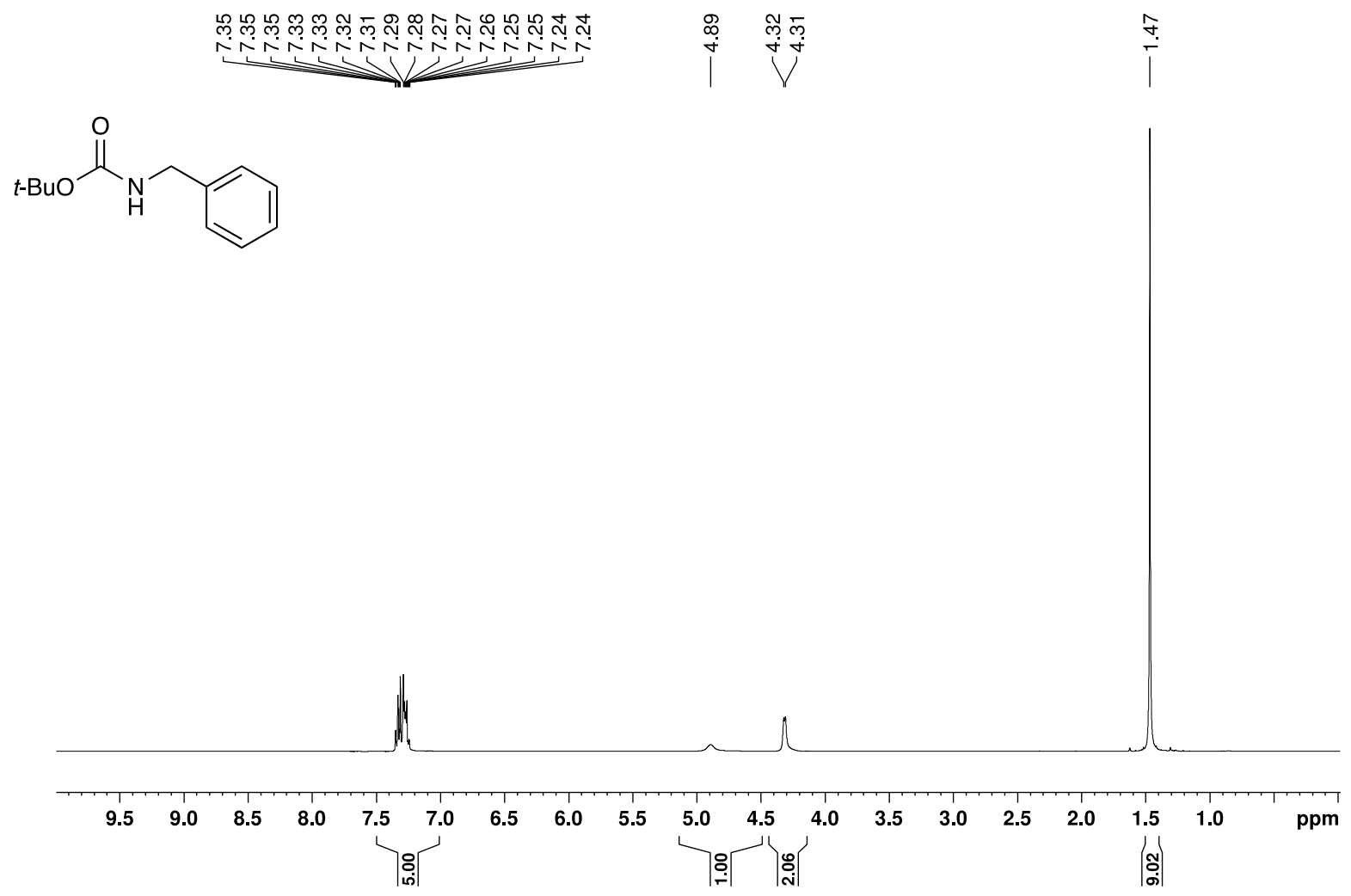

${ }^{13} \mathrm{C}$ NMR $\left(101 \mathrm{MHz}, \mathrm{CDCl}_{3}\right)$

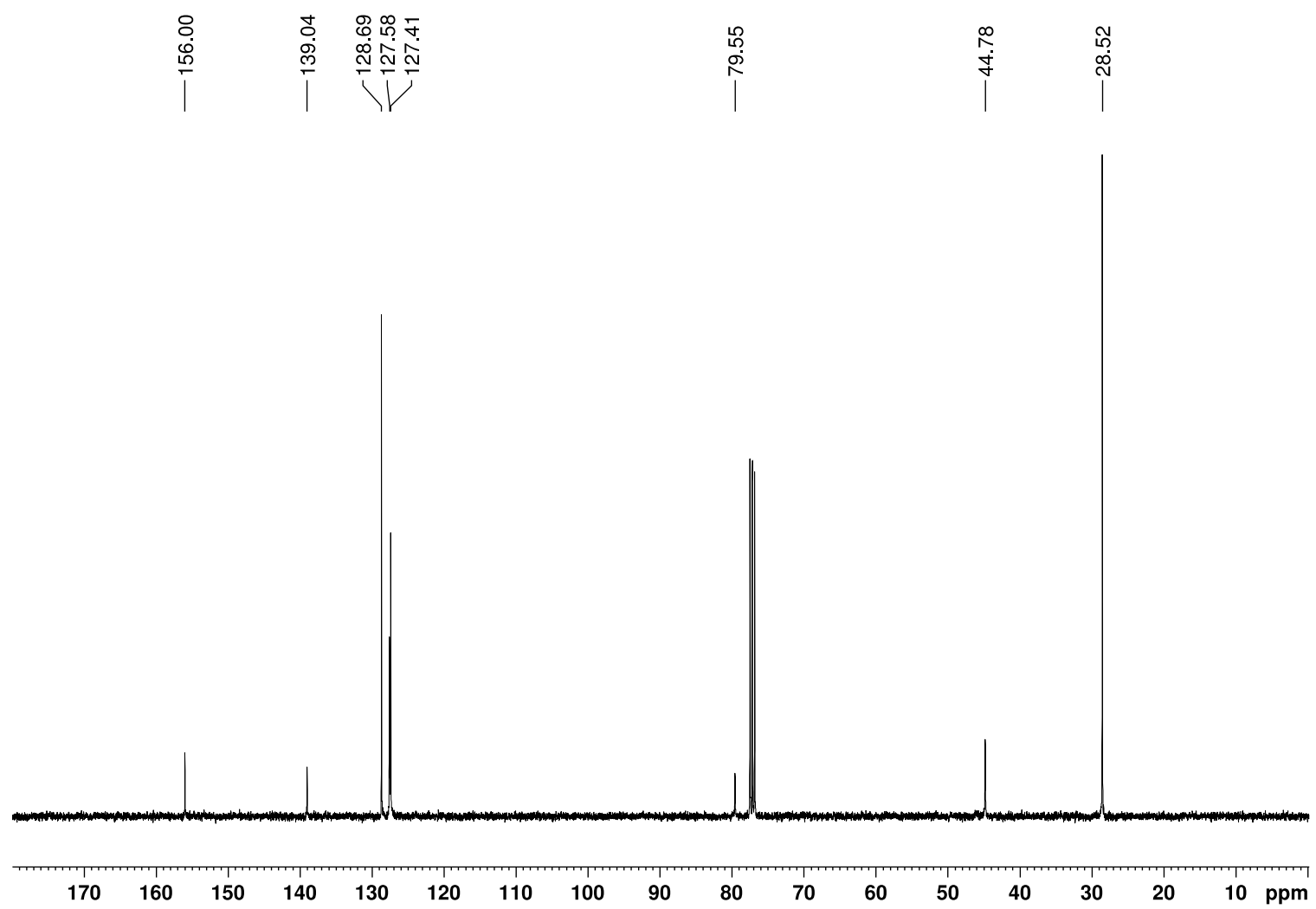


$\mathbf{N}$-Boc, $\mathbf{N}$-benzyl-allylamine, $1 \mathbf{1 a}$

${ }^{1} \mathrm{H}$ NMR (400 MHz, $\mathrm{CDCl}_{3}$ )

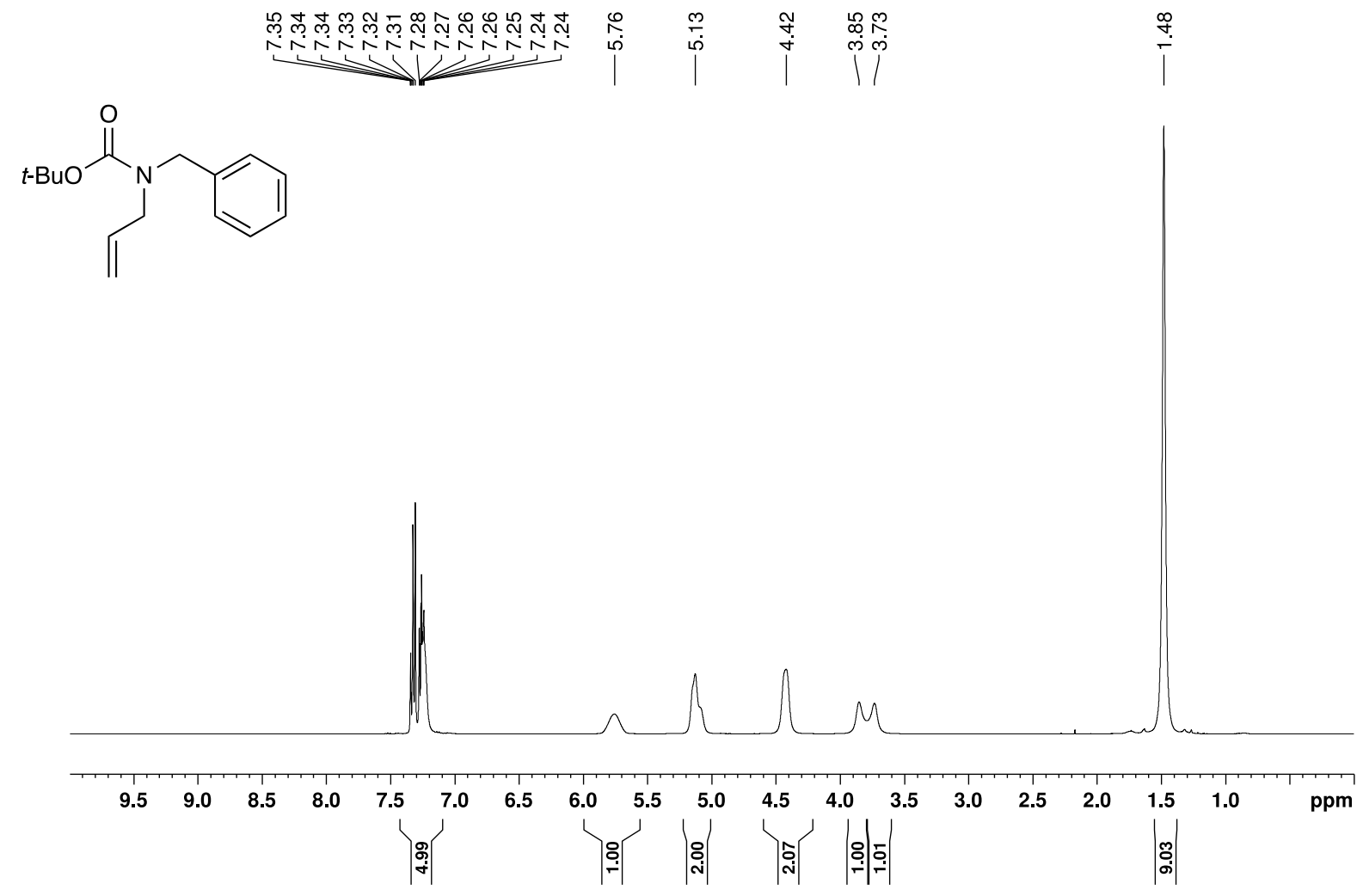

${ }^{13} \mathrm{C}$ NMR (101 MHz, CDCl $)$

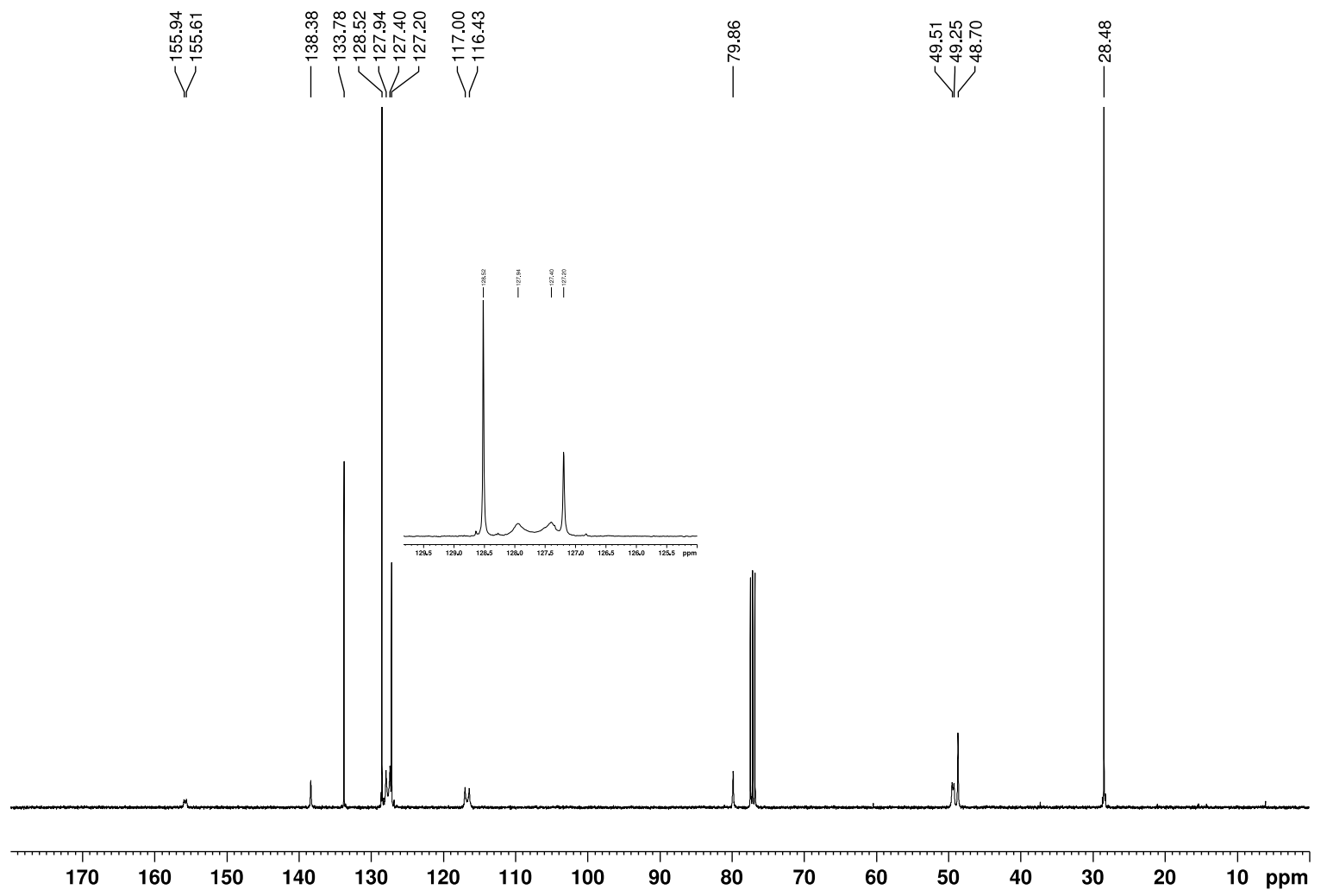


(+)-N-Boc-[(R)-phenylethyl]-amine, 1b' ${ }^{1} \mathrm{H} \mathrm{NMR}\left(400 \mathrm{MHz}, \mathrm{CDCl}_{3}\right)$
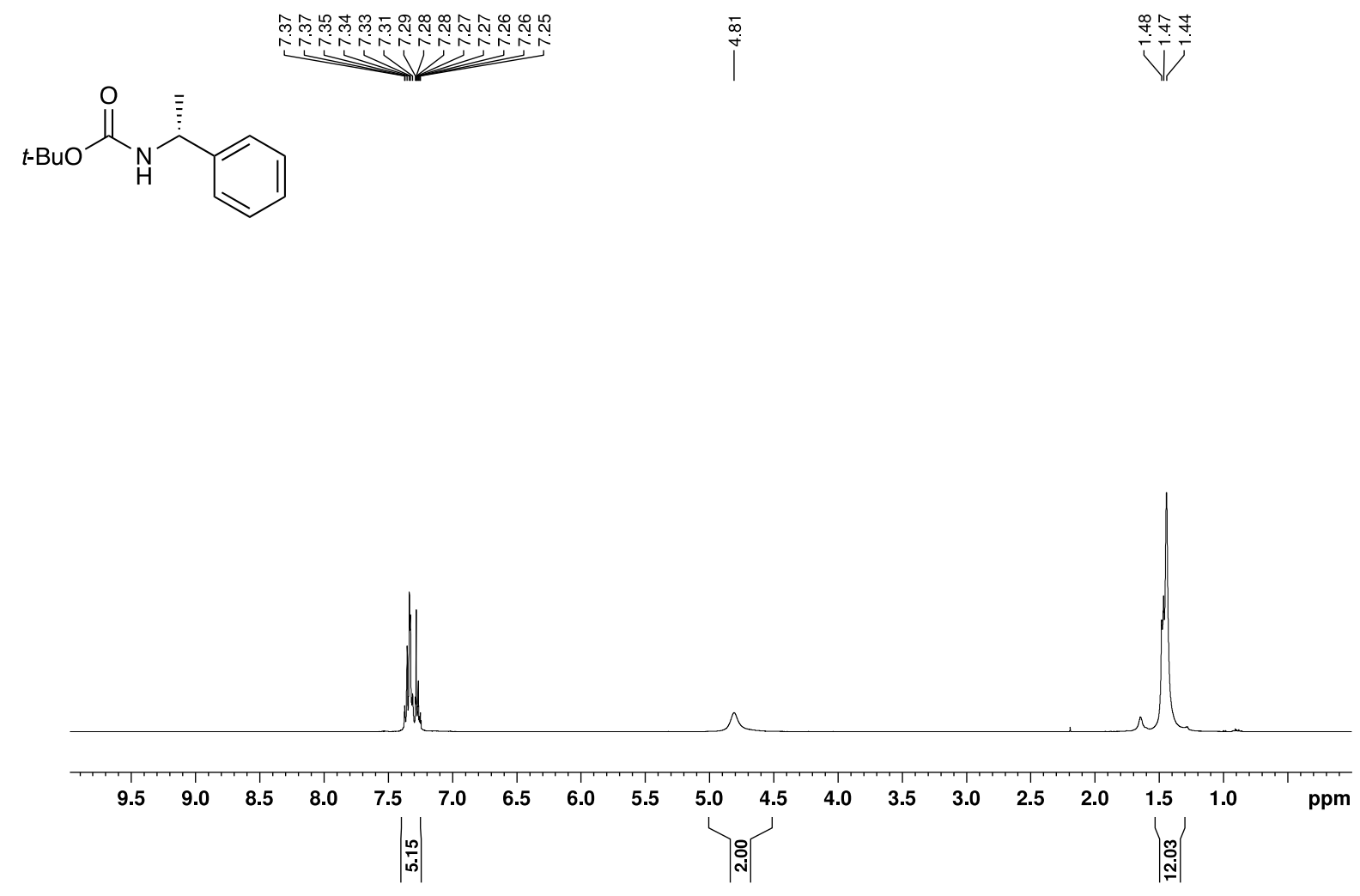

${ }^{13} \mathrm{C}$ NMR (101 MHz, $\mathrm{CDCl}_{3}$ )

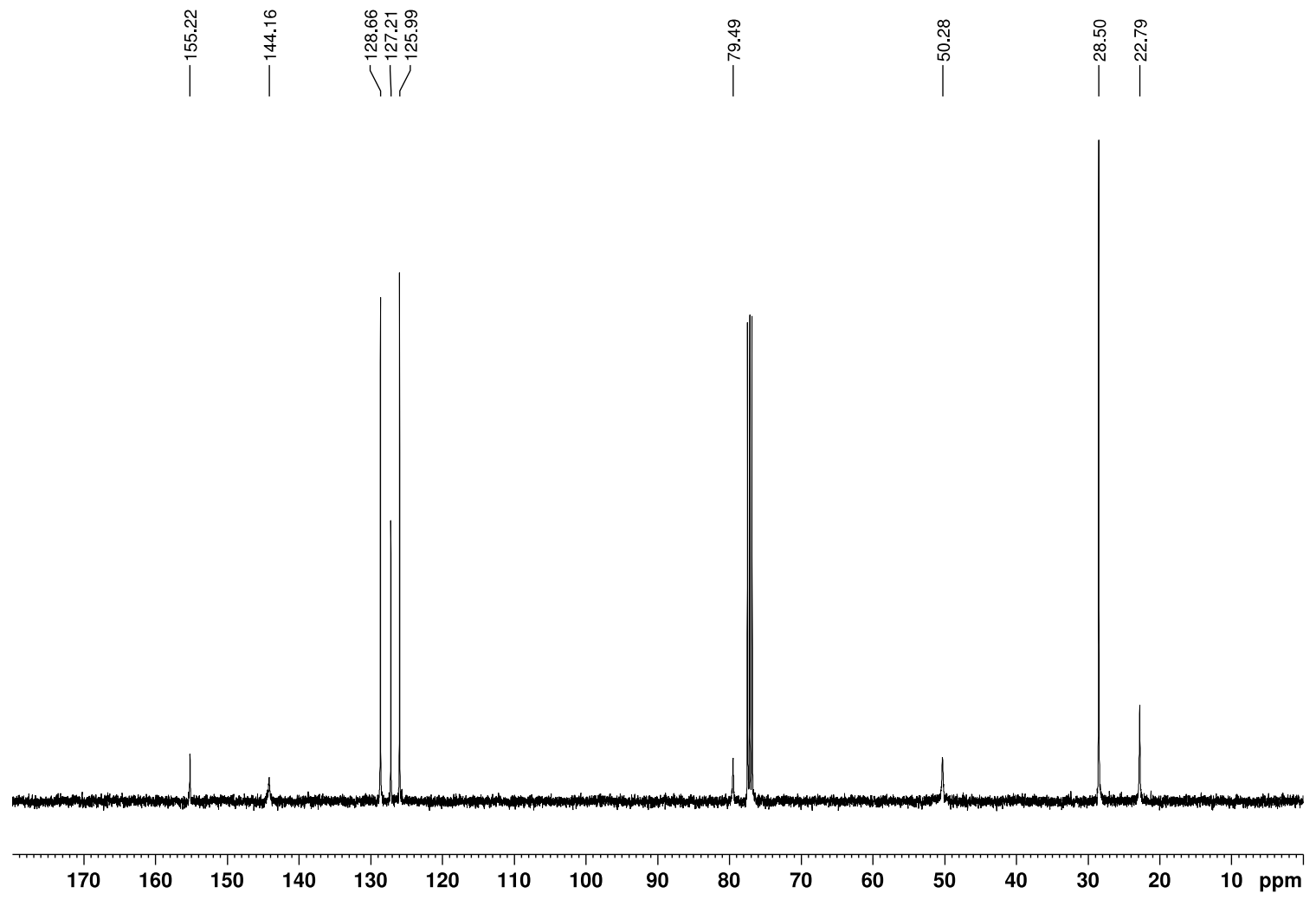


(+)-N-Boc, $\mathrm{N}$-[(R)-phenylethyl]-allylamine, $\mathbf{1 b}$

${ }^{1} \mathrm{H}$ NMR (400 MHz, $\mathrm{CDCl}_{3}$ )

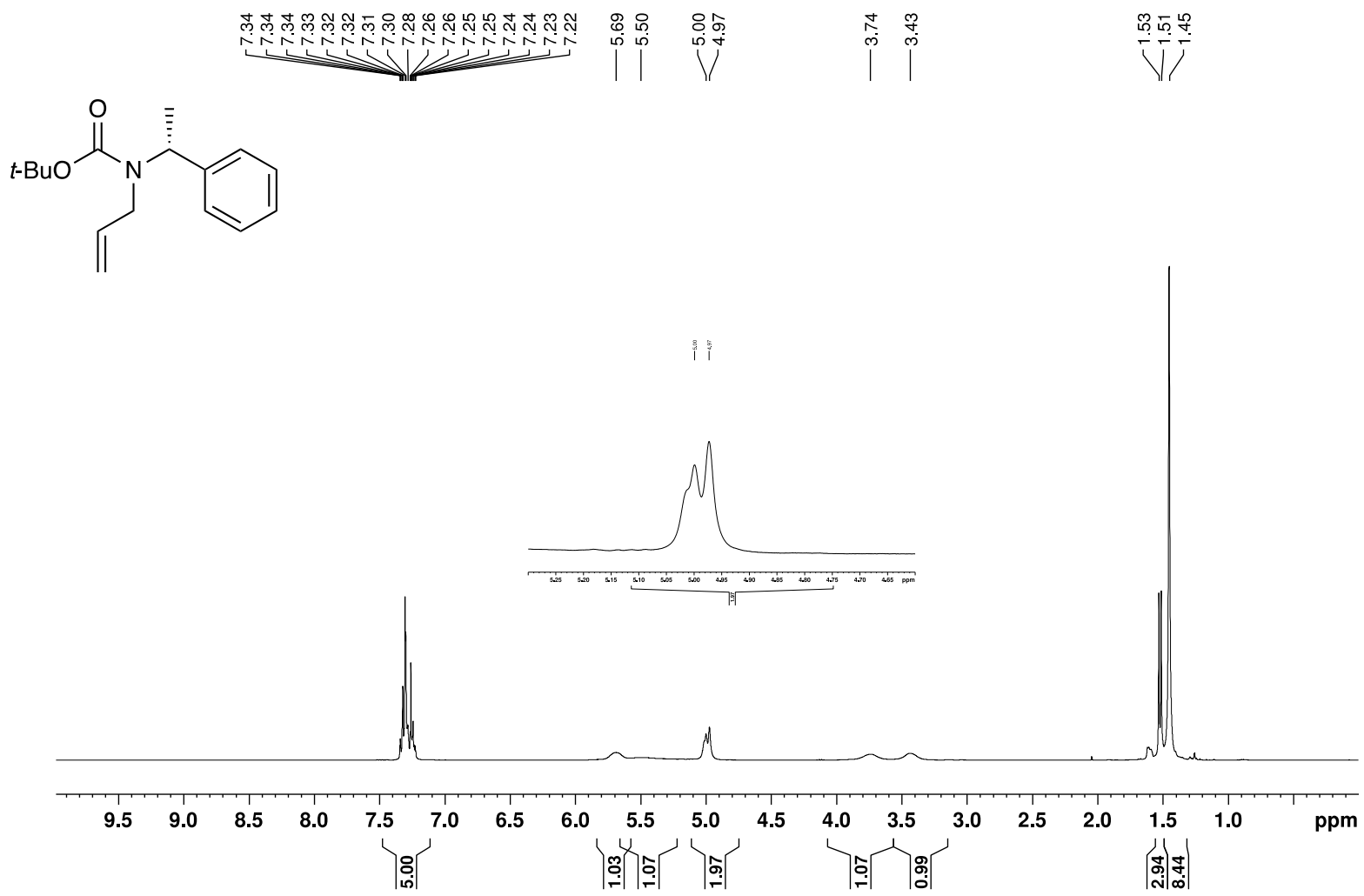

${ }^{13} \mathrm{C}$ NMR (101 MHz, $\mathrm{CDCl}_{3}$ )

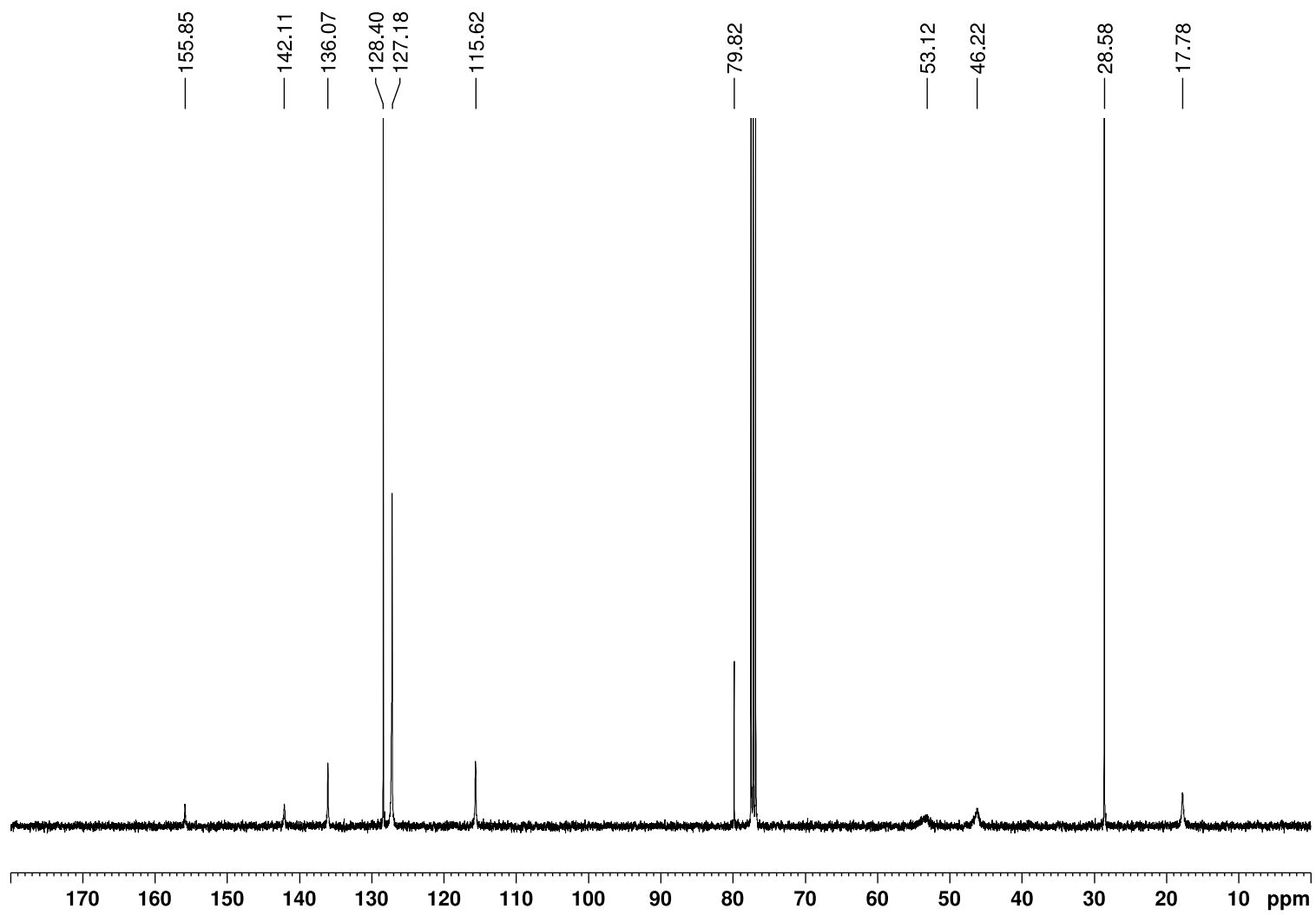


(士)-N-Benzyl-cyclohex-2-ene-1-amine, 1c'

${ }^{1} \mathrm{H} \mathrm{NMR}\left(400 \mathrm{MHz}, \mathrm{CDCl}_{3}\right)$

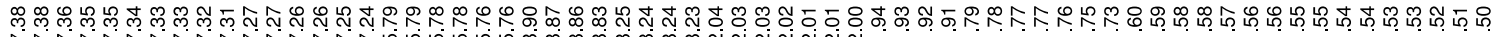

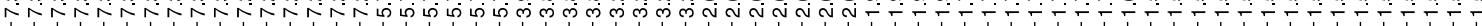<smiles>C1=CC(NCc2ccccc2)CCC1</smiles>

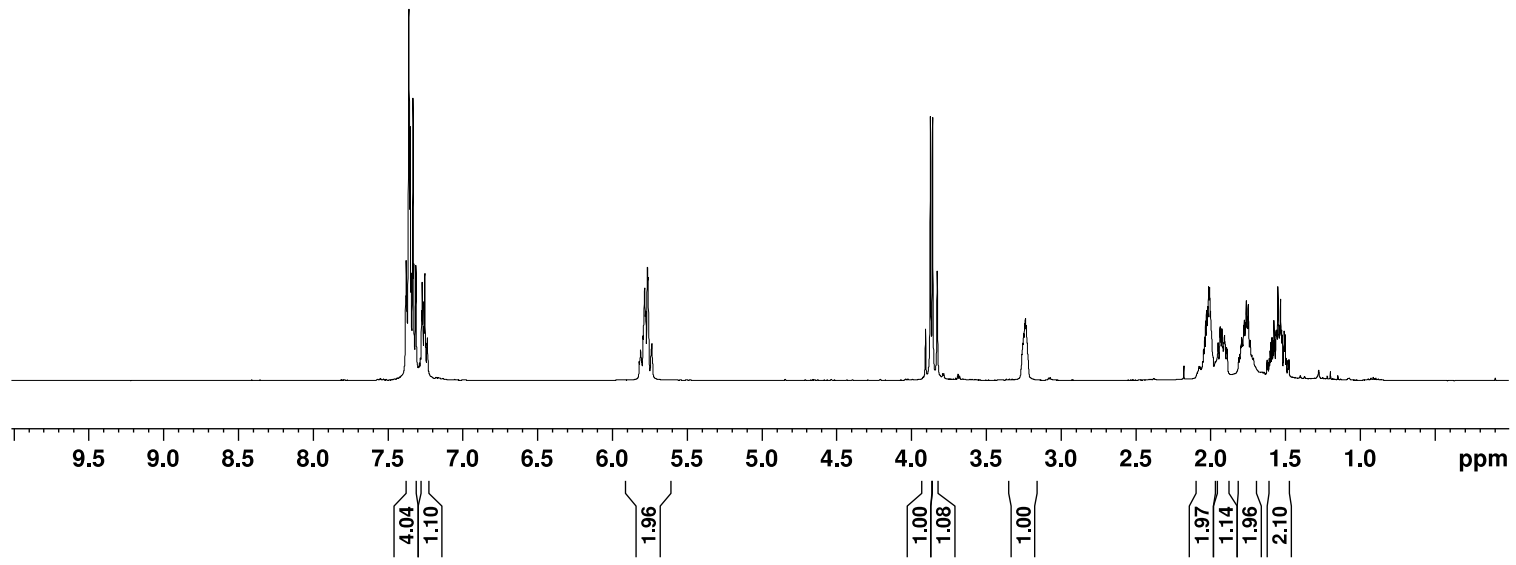

${ }^{13} \mathrm{C} \mathrm{NMR}\left(101 \mathrm{MHz}, \mathrm{CDCl}_{3}\right)$

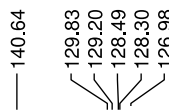

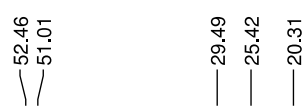
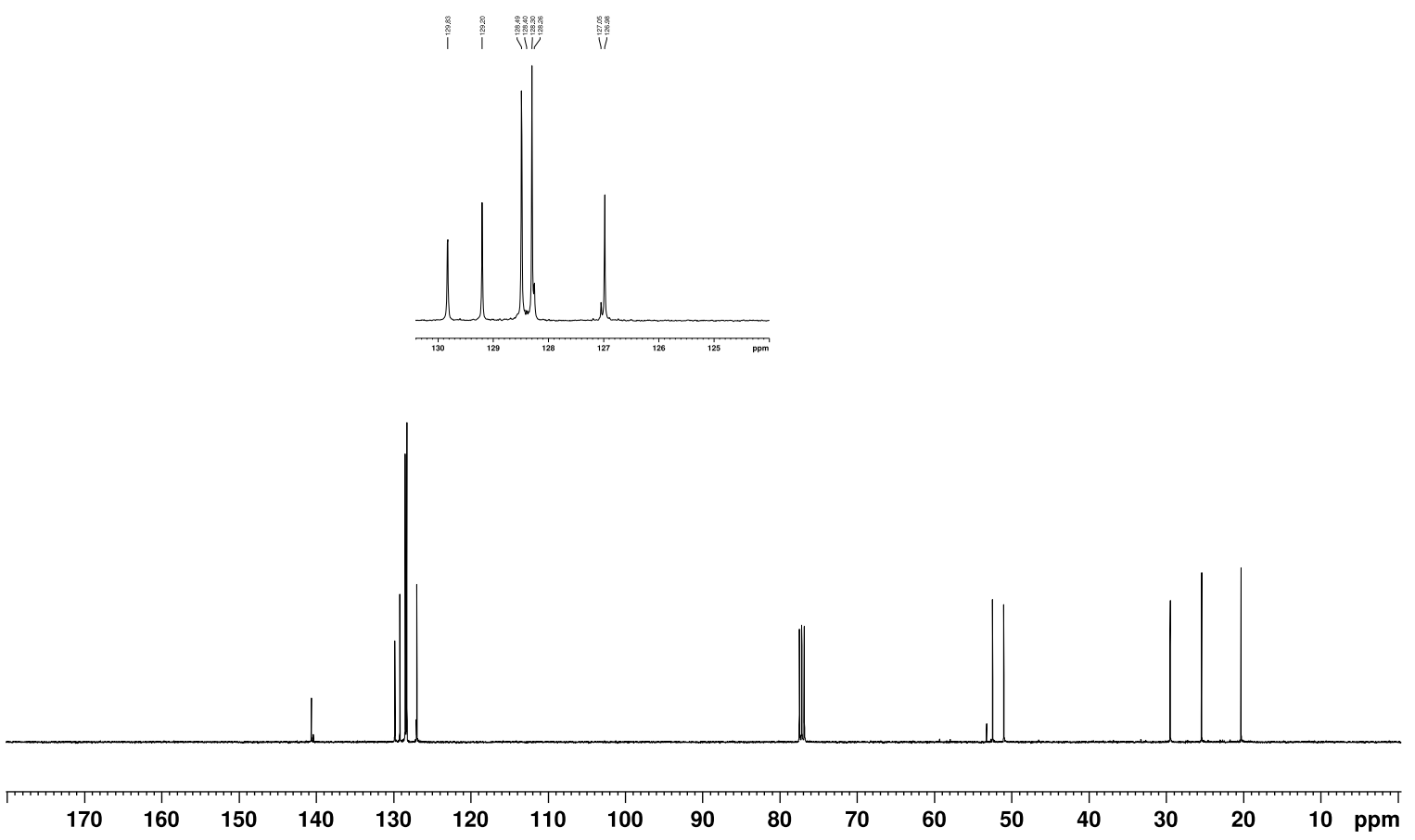
( \pm )-N-Boc, $N$-benzyl-cyclohex-2-ene-1-amine, 1c ${ }^{1} \mathrm{H} \mathrm{NMR}\left(400 \mathrm{MHz}, \mathrm{CDCl}_{3}\right)$
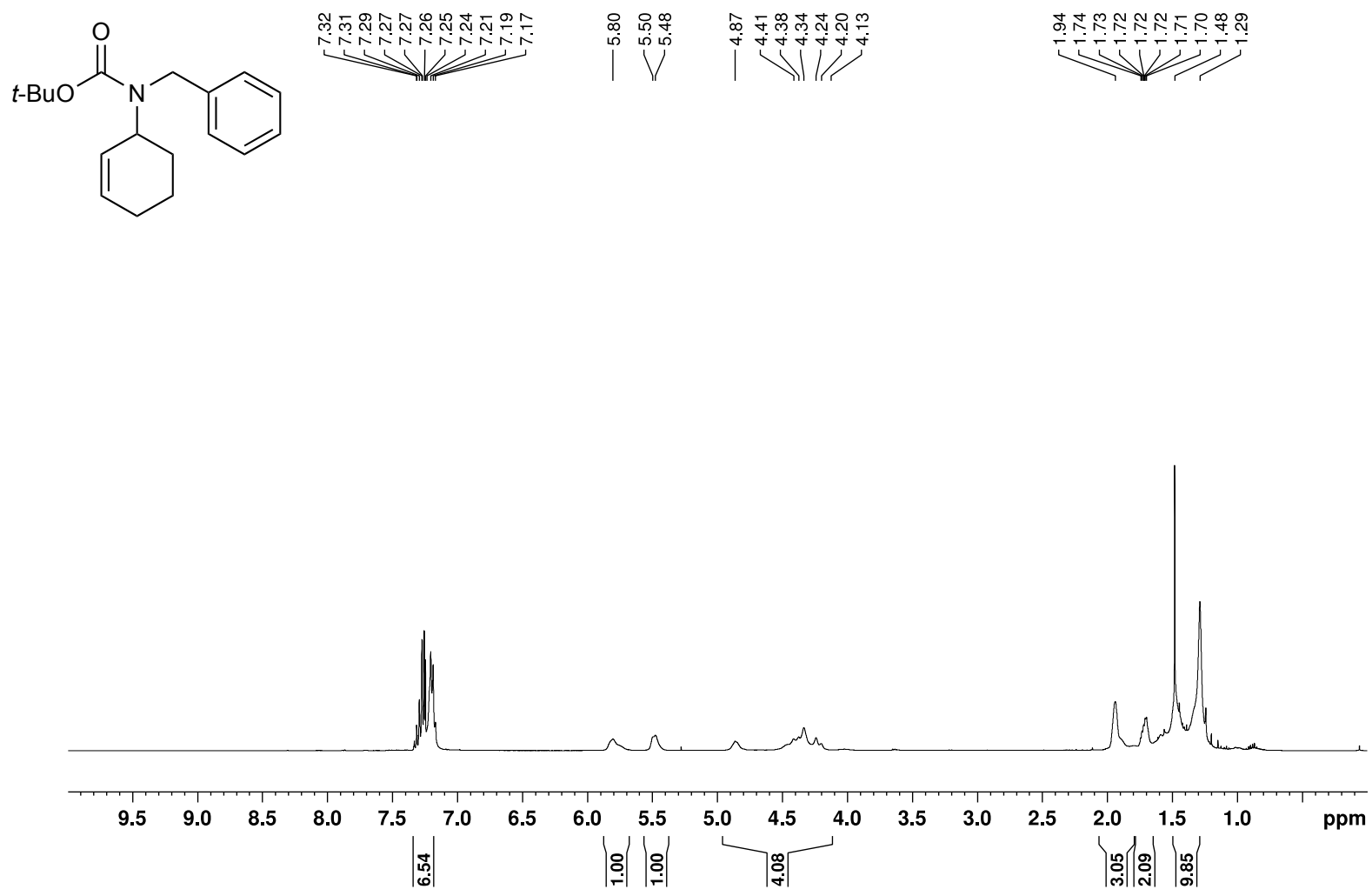

${ }^{13} \mathrm{C}$ NMR $\left(101 \mathrm{MHz}, \mathrm{CDCl}_{3}\right)$

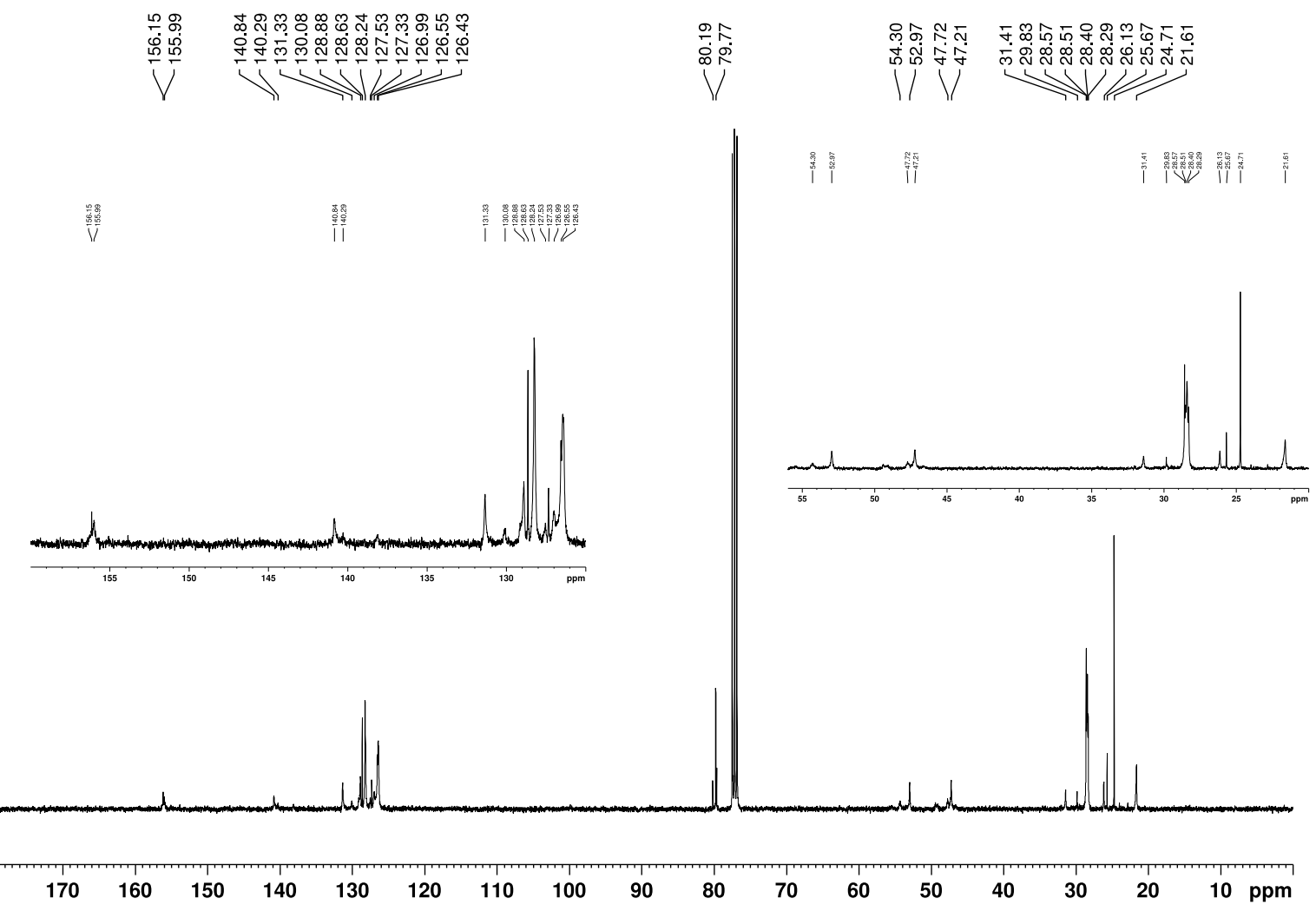


HSQC 2D-NMR $\left({ }^{13} \mathrm{C} /{ }^{1} \mathrm{H}, \mathrm{CDCl}_{3}\right)$

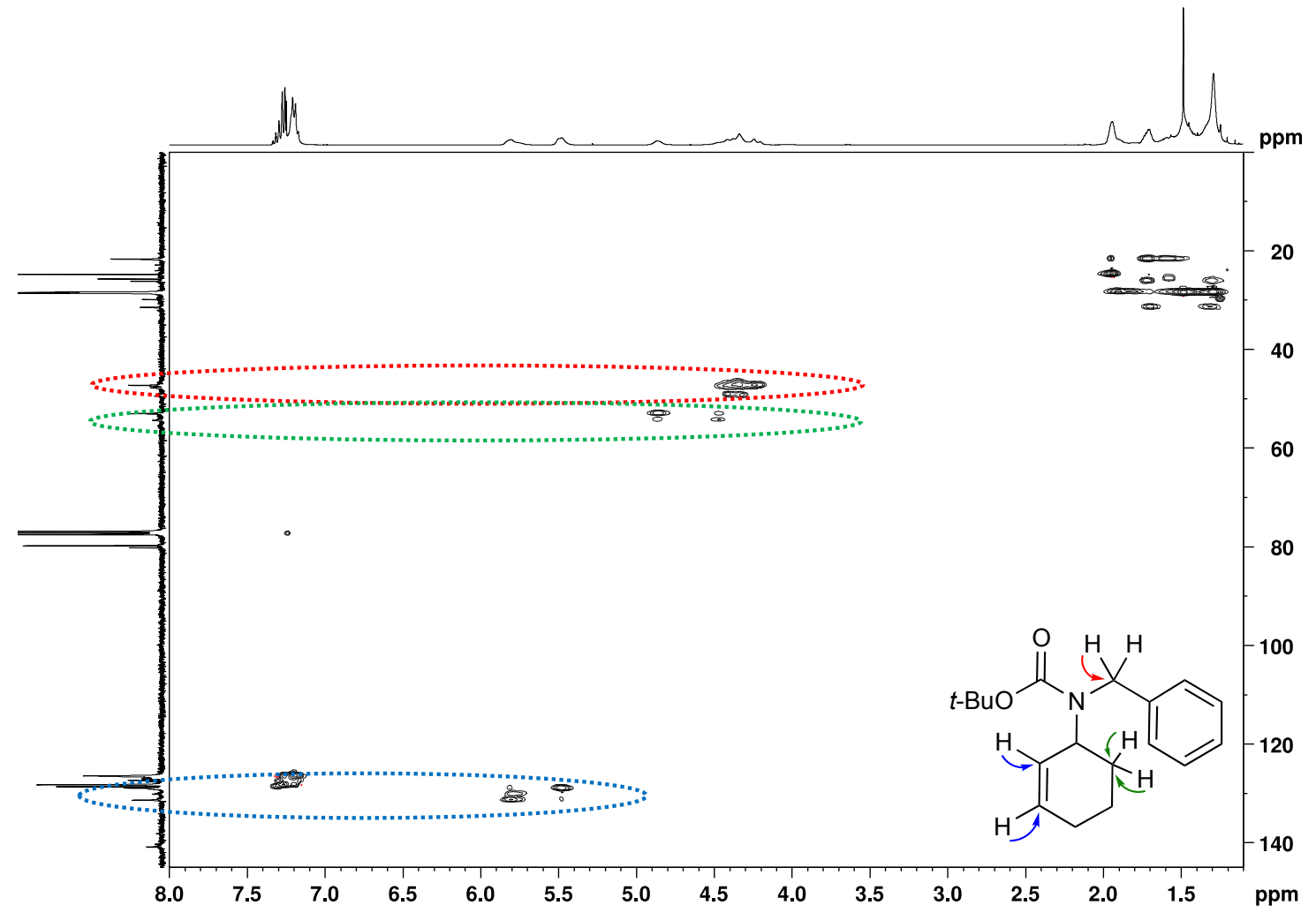


(-)-(S)-N-Boc-2-hydroxymethylpyrrolidine, 1d'

${ }^{1} \mathrm{H} \mathrm{NMR}\left(400 \mathrm{MHz}, \mathrm{CDCl}_{3}\right)$<smiles>CC(C)(C)OC(=O)N1CCC[C@H]1CO</smiles>

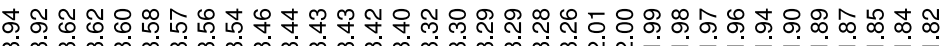

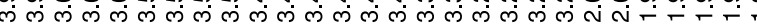

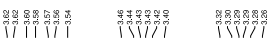
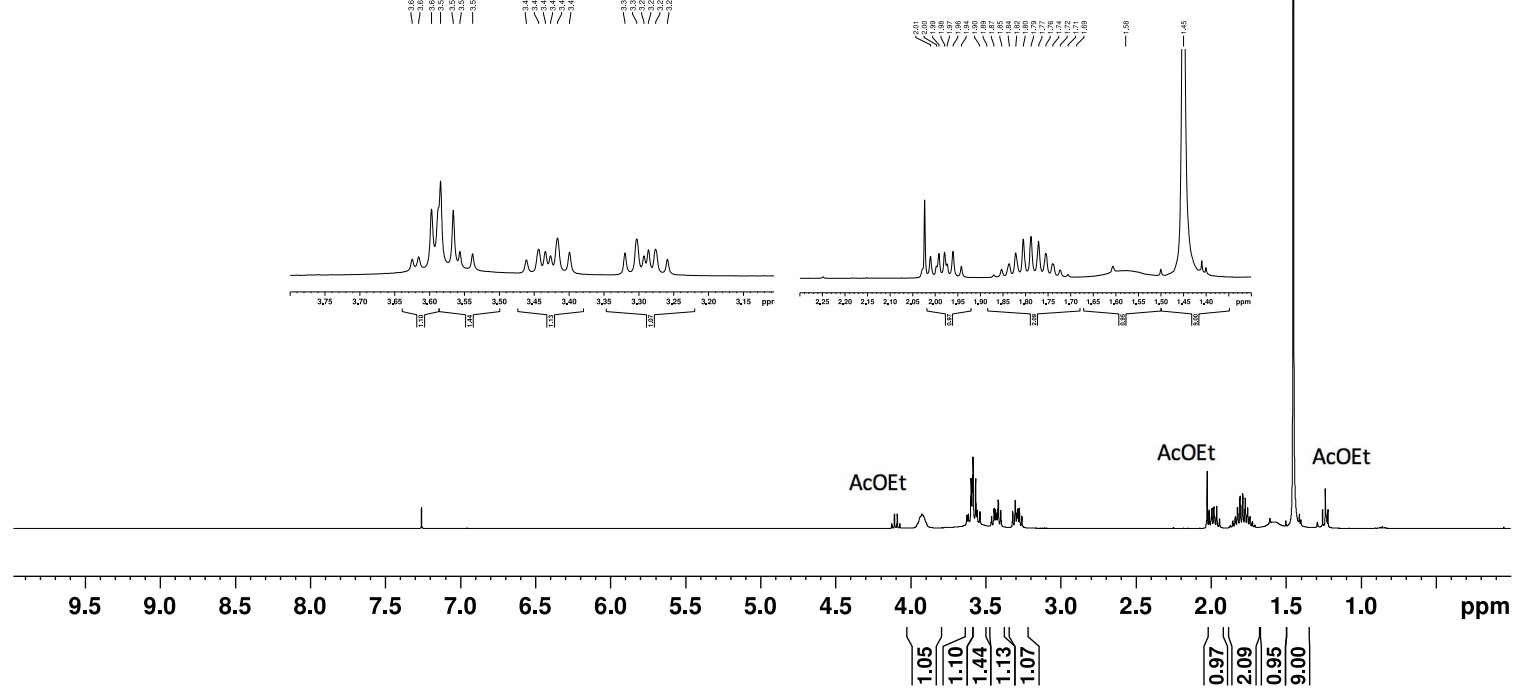

${ }^{13} \mathrm{C}$ NMR $\left(101 \mathrm{MHz}, \mathrm{CDCl}_{3}\right)$

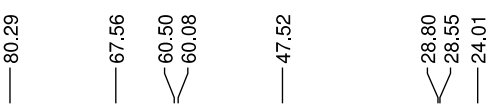

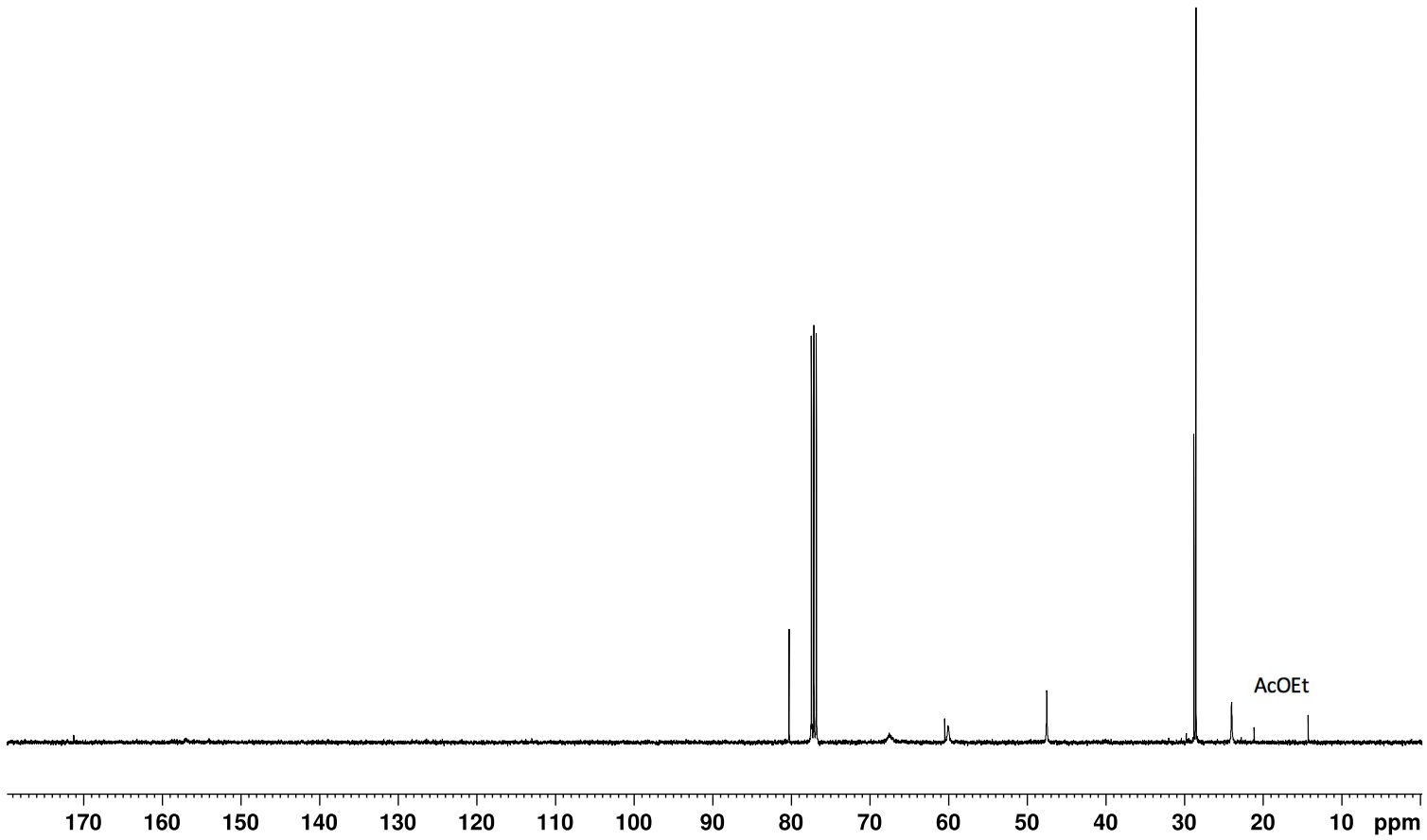


(-)-(S)-N-Boc-2-formylpyrrolidine, 1d" ${ }^{1} \mathrm{H} \mathrm{NMR}\left(400 \mathrm{MHz}, \mathrm{CDCl}_{3}\right)$

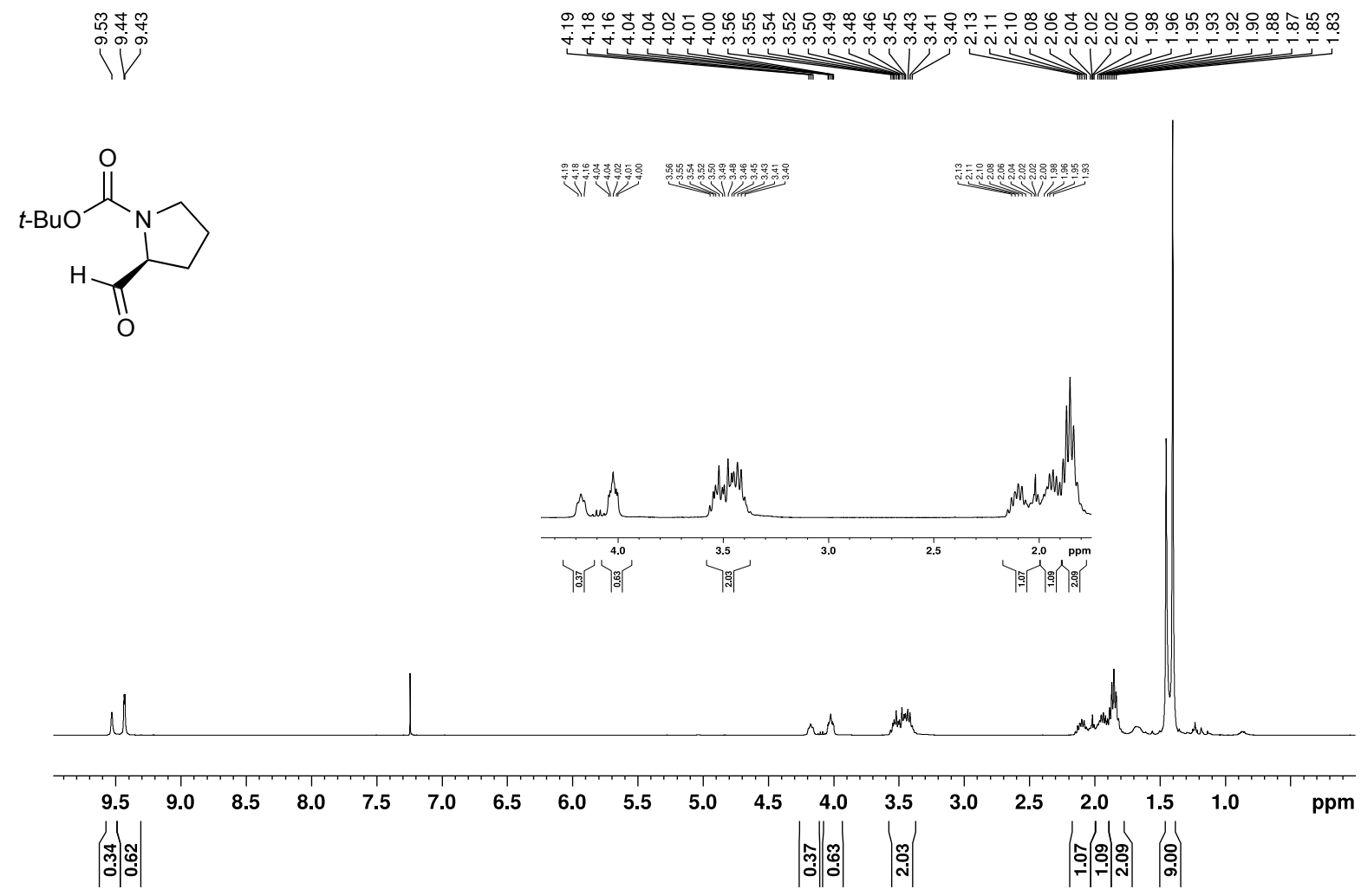

${ }^{13} \mathrm{C}$ NMR (101 MHz, CDCl $)$

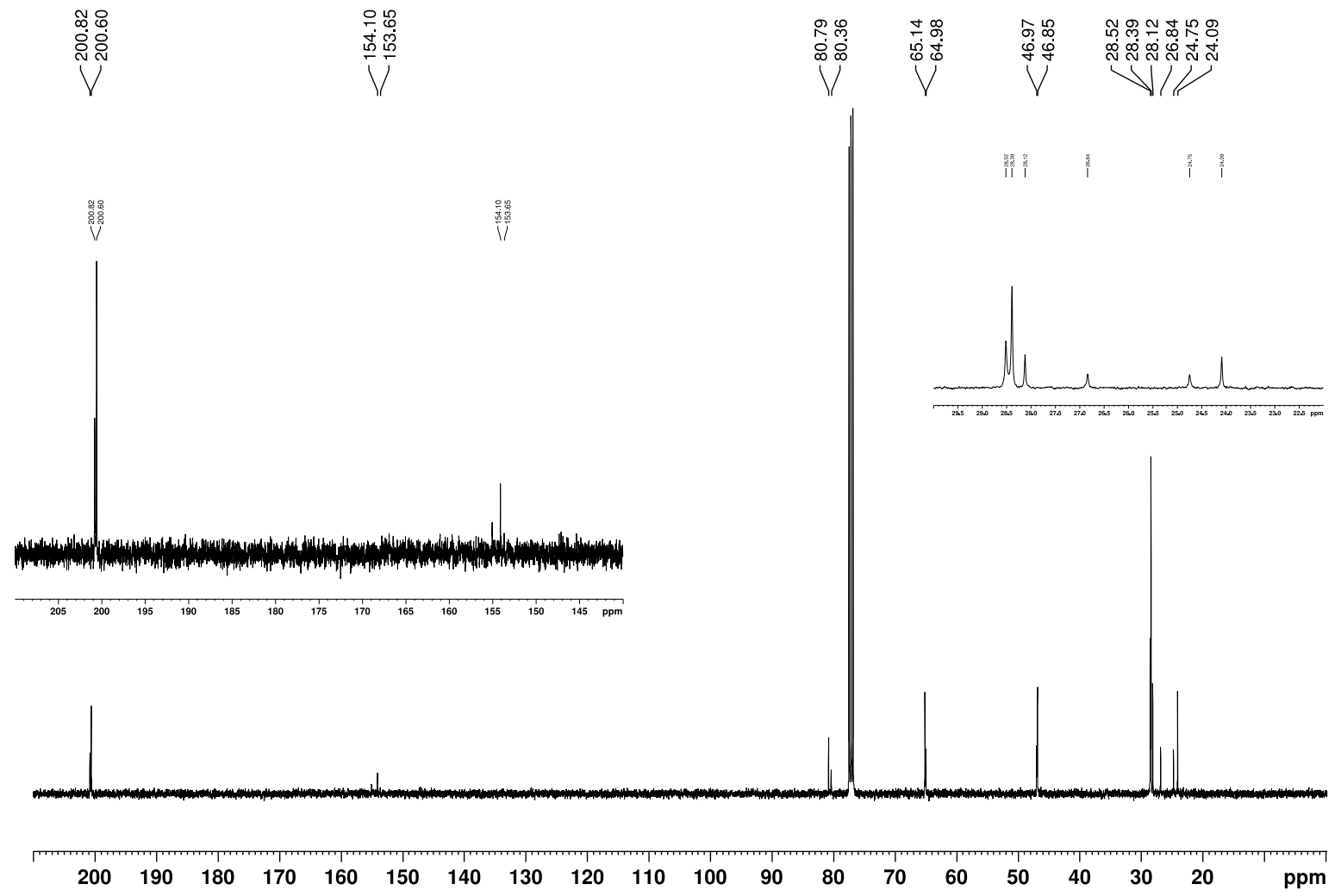


(-)-(S)-N-Boc-2-vinylpyrrolidine, 1d

${ }^{1} \mathrm{H}$ NMR $\left(400 \mathrm{MHz}, \mathrm{CDCl}_{3}\right)$

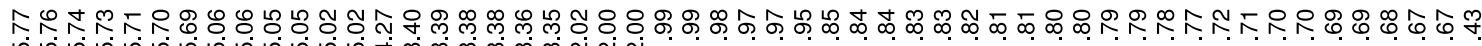<smiles>C=C[C@H]1CCCN1C(=O)OC(C)(C)C</smiles>
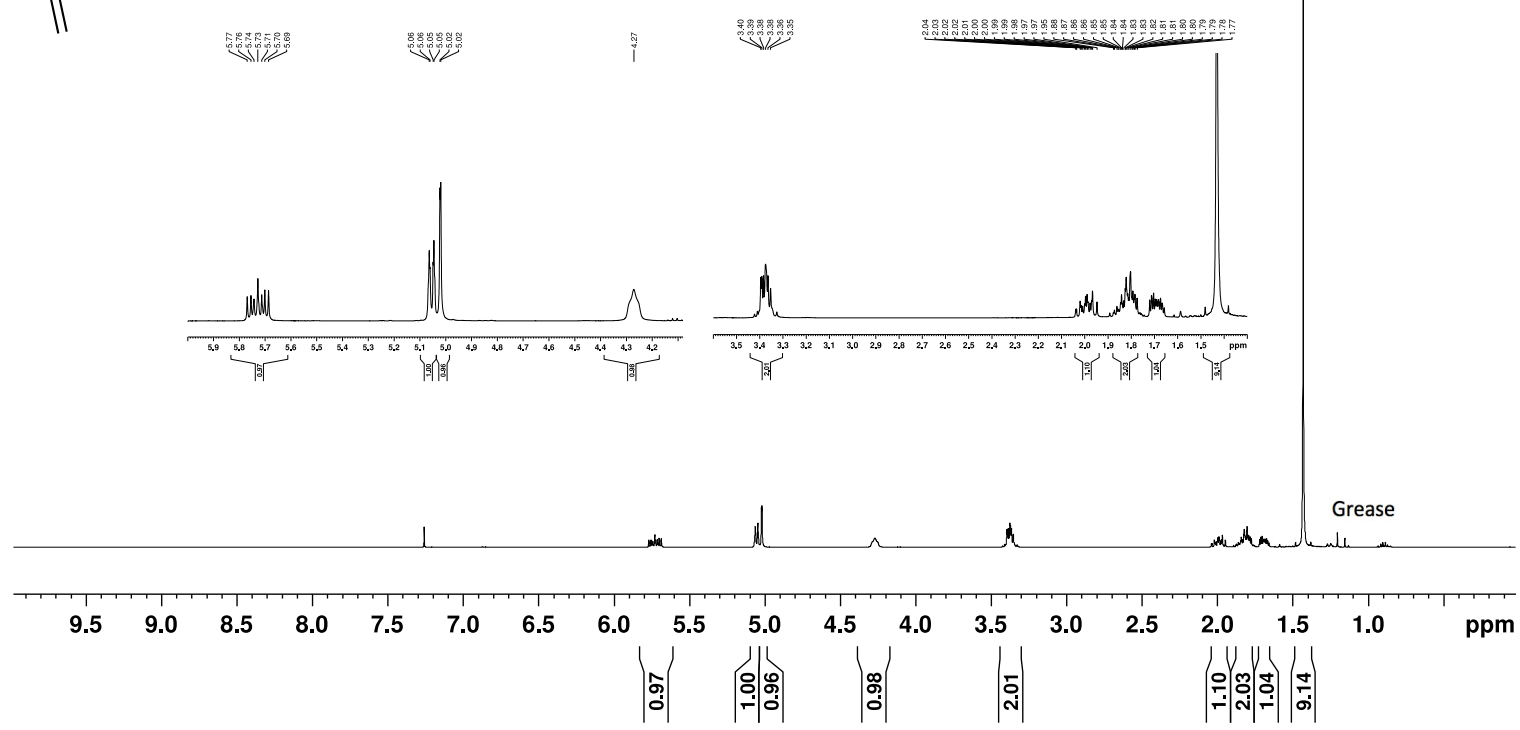

${ }^{13} \mathrm{C}$ NMR (101 MHz, $\left.\mathrm{CDCl}_{3}\right)$

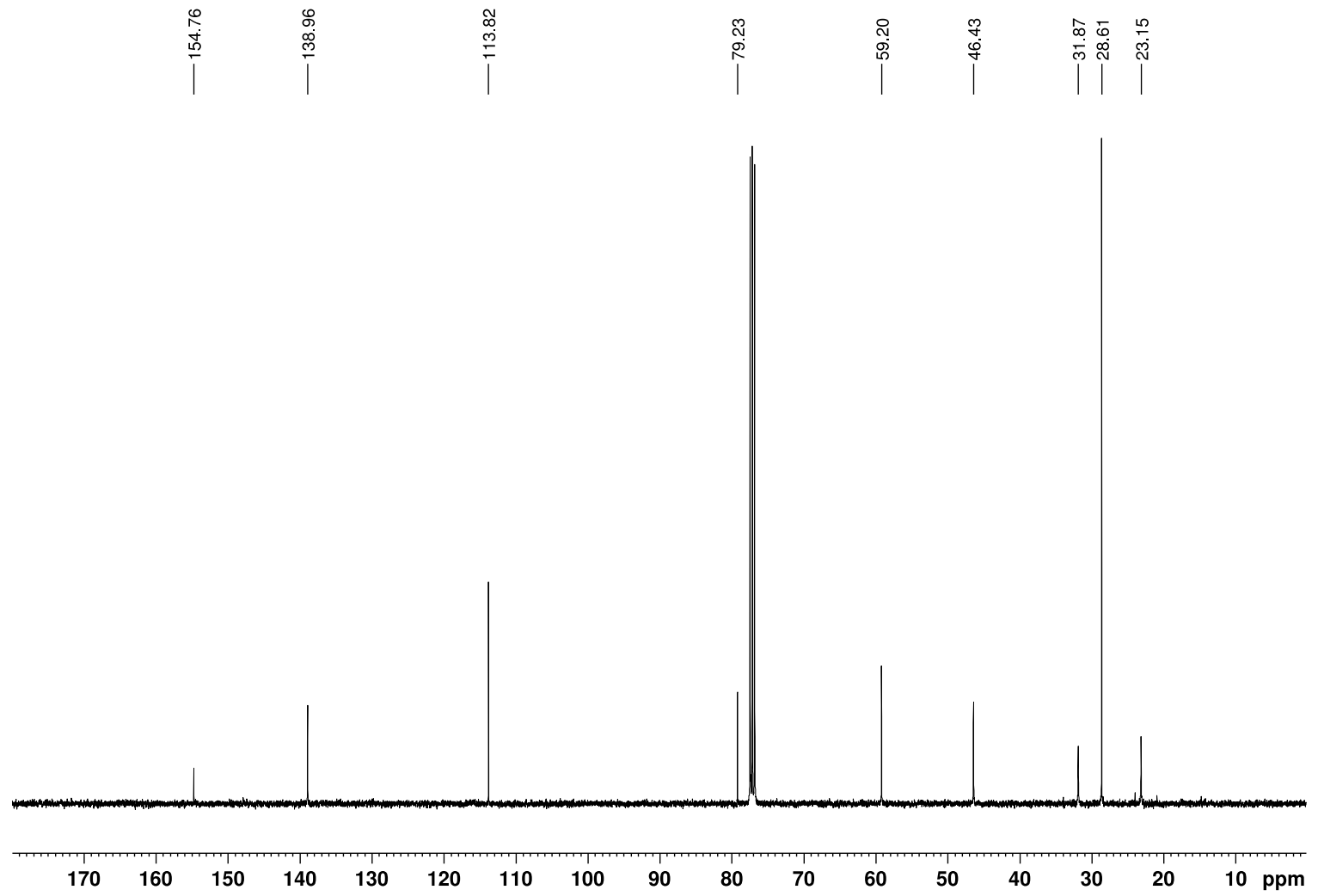


( \pm )-N-Boc-2-hydroxymethylpiperidine, 1e' ${ }^{1} \mathrm{H} \mathrm{NMR}\left(400 \mathrm{MHz}, \mathrm{CDCl}_{3}\right)$<smiles>CC(C)(C)OC(=O)N1CCCCC1CO</smiles>
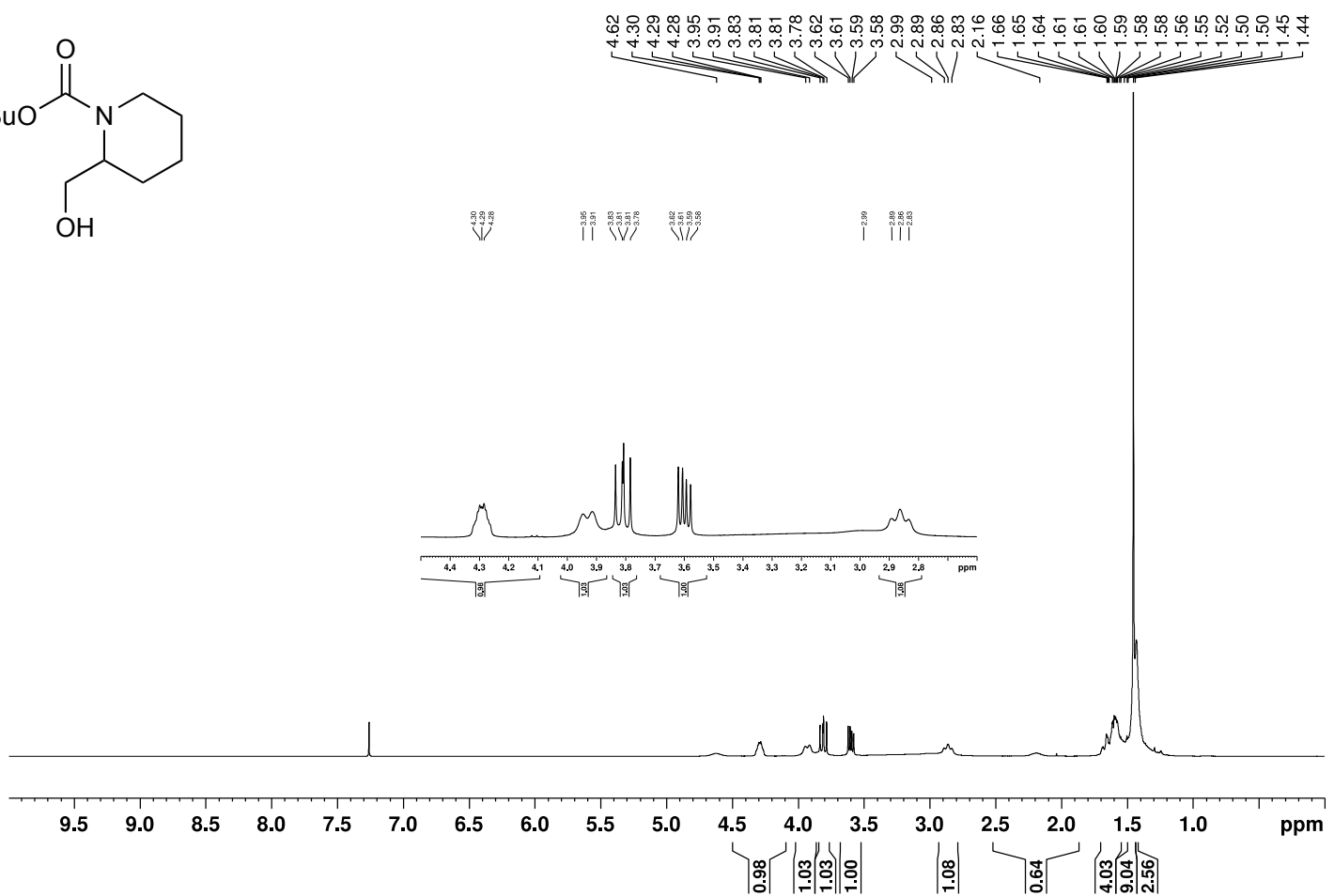

${ }^{13} \mathrm{C}$ NMR (101 MHz, $\mathrm{CDCl}_{3}$ )

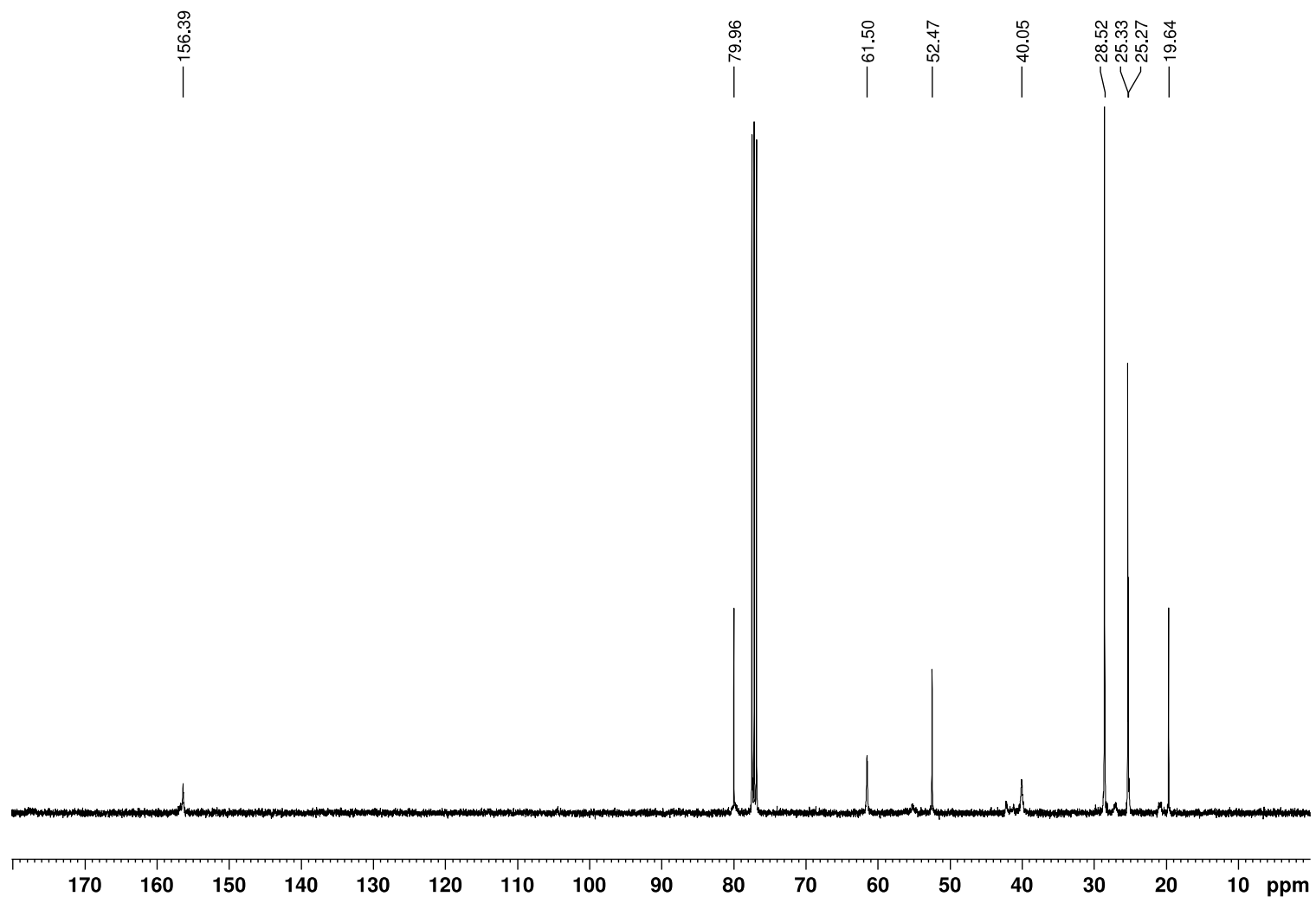


( \pm )-N-Boc-2-formylpiperidine, 1e" ${ }^{1} \mathrm{H} \mathrm{NMR}\left(400 \mathrm{MHz}, \mathrm{CDCl}_{3}\right)$<smiles>CC(C)(C)OC(=O)N1CCCCC1C=O</smiles>

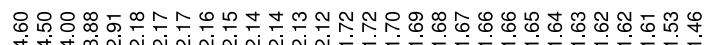

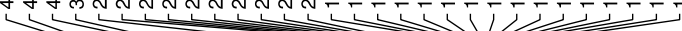

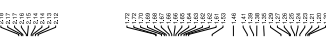
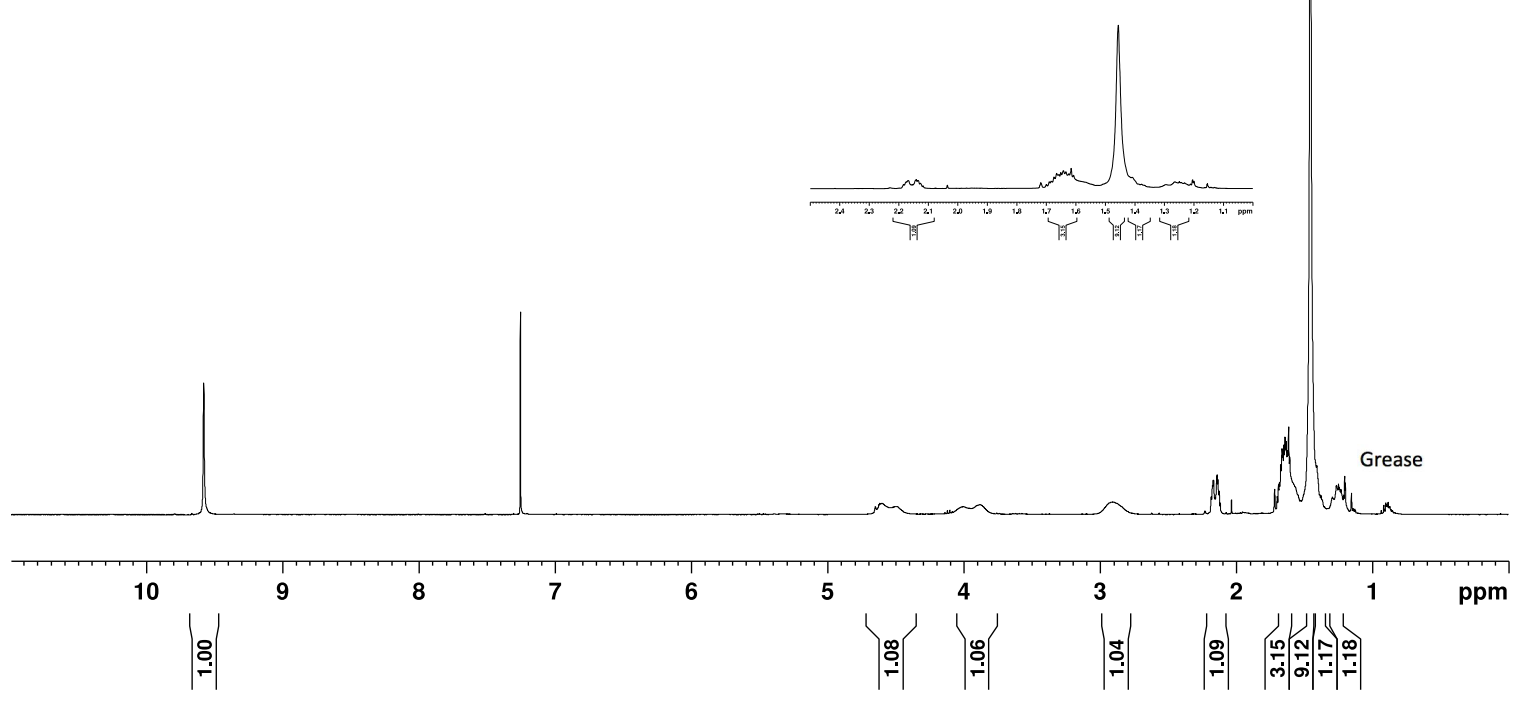

$\left.{ }^{13} \mathrm{C} \mathrm{NMR} \mathrm{(101} \mathrm{MHz,} \mathrm{CDCl}_{3}\right)$

I

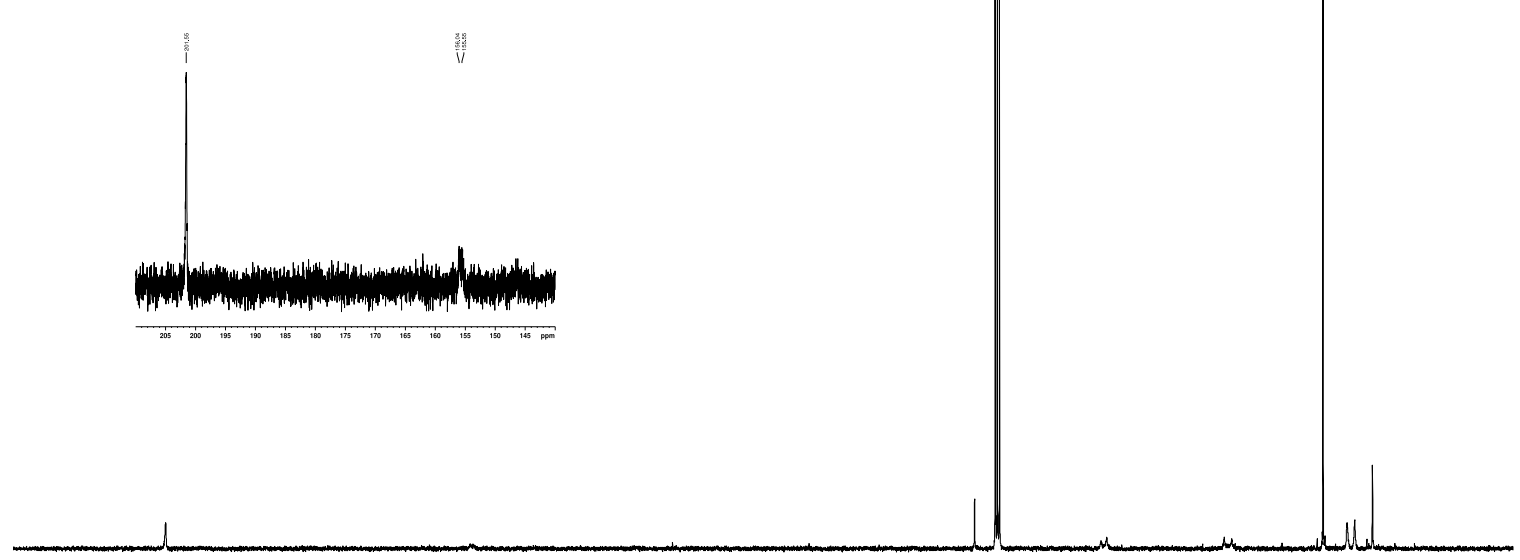

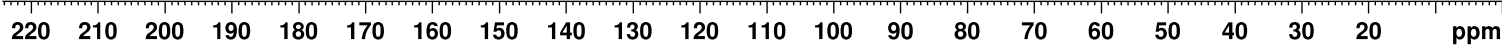


HSQC 2D-NMR $\left({ }^{13} \mathrm{C} /{ }^{1} \mathrm{H}, \mathrm{CDCl}_{3}\right)$

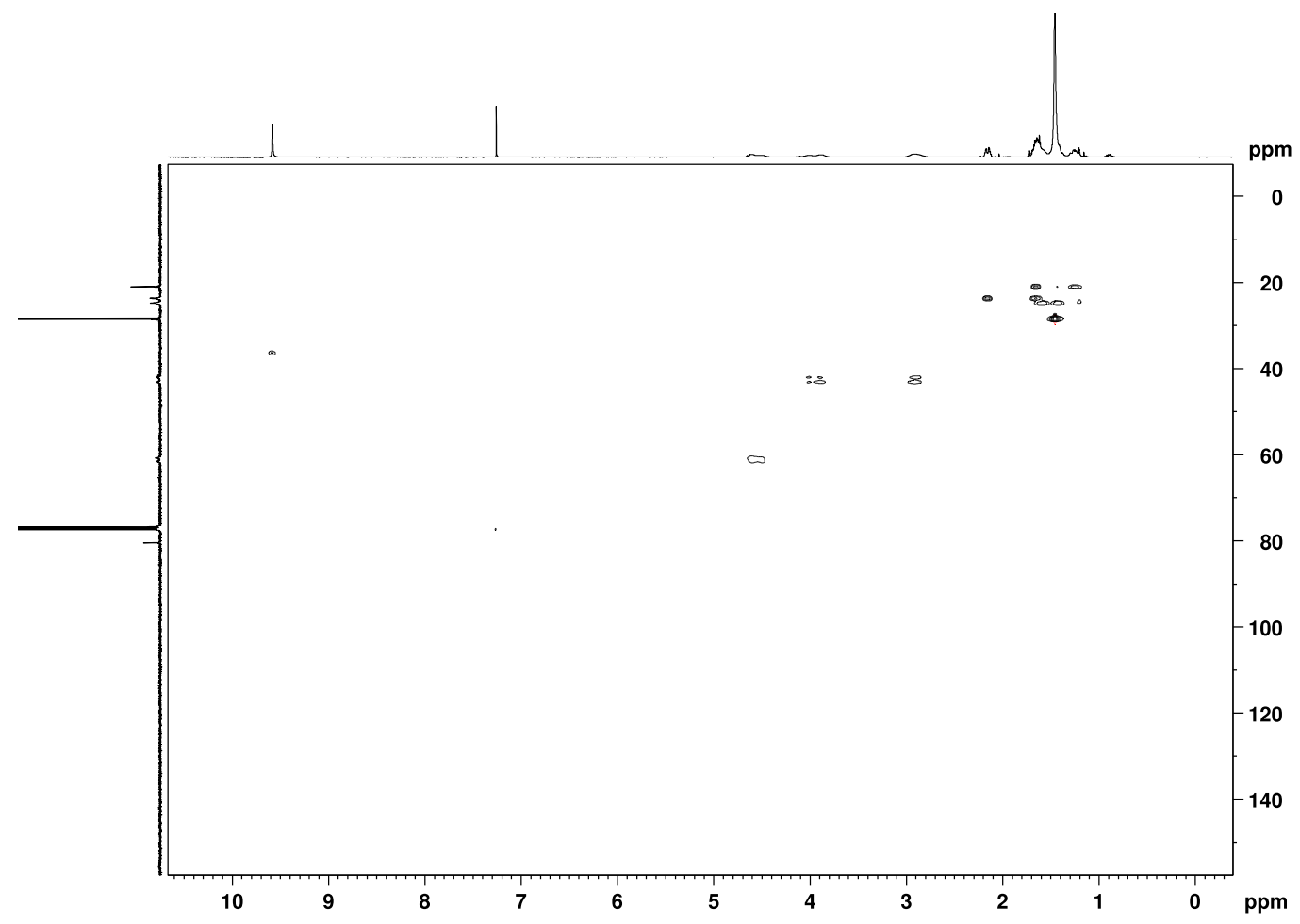

HSQC 2D-NMR $\left({ }^{13} \mathrm{C} /{ }^{1} \mathrm{H}, \mathrm{CDCl}_{3}\right)$ - Zoom

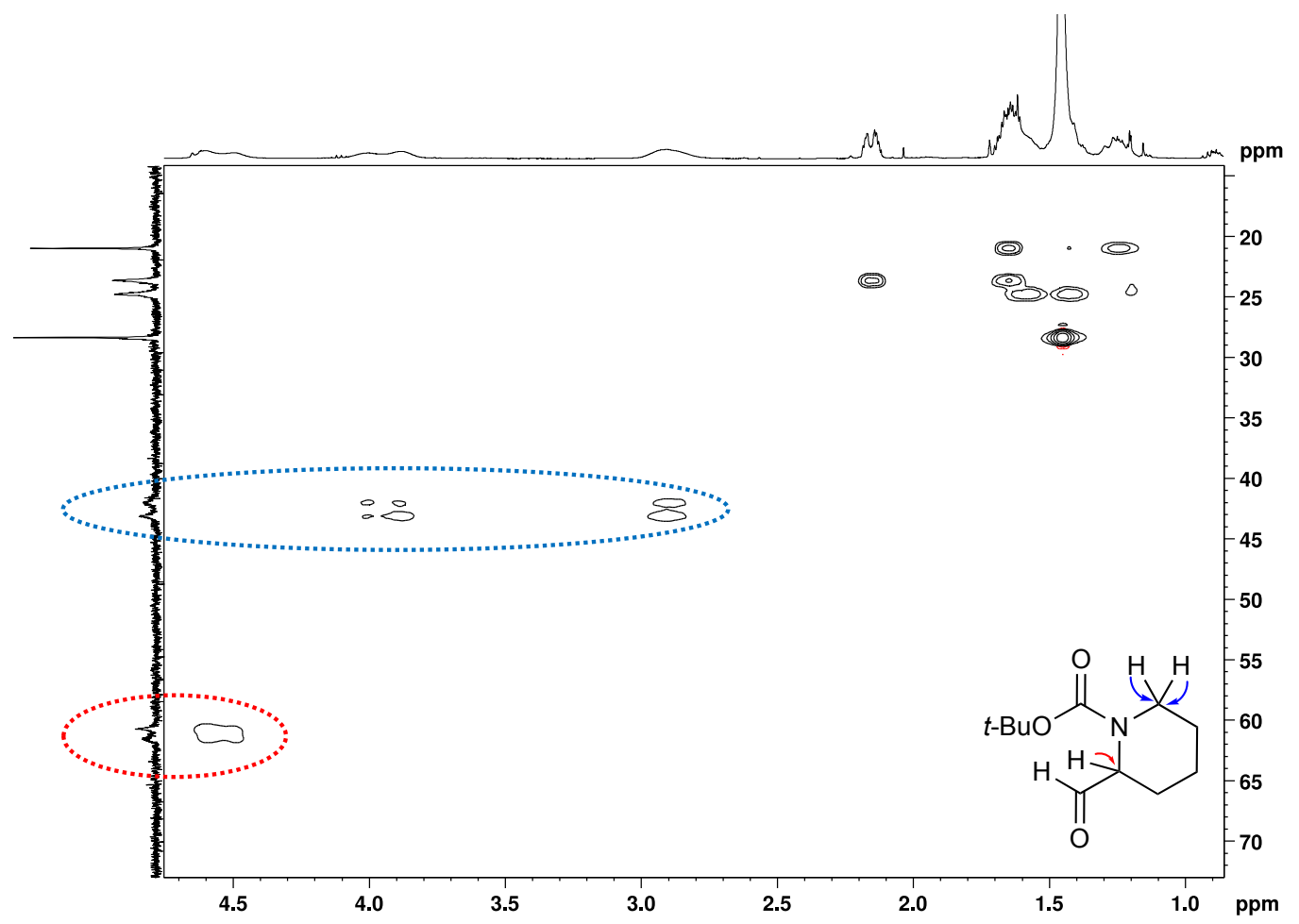


( \pm )-N-Boc-2-vinylpiperidine, 1e

$\left.{ }^{1} \mathrm{H} \mathrm{NMR} \mathrm{(400} \mathrm{MHz,} \mathrm{CDCl}_{3}\right)$

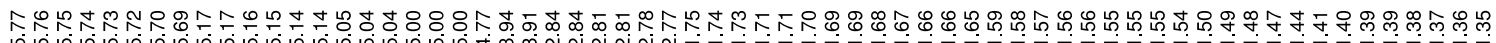

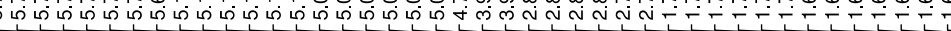<smiles>C=CC1CCCCN1C(=O)OC(C)(C)C</smiles>
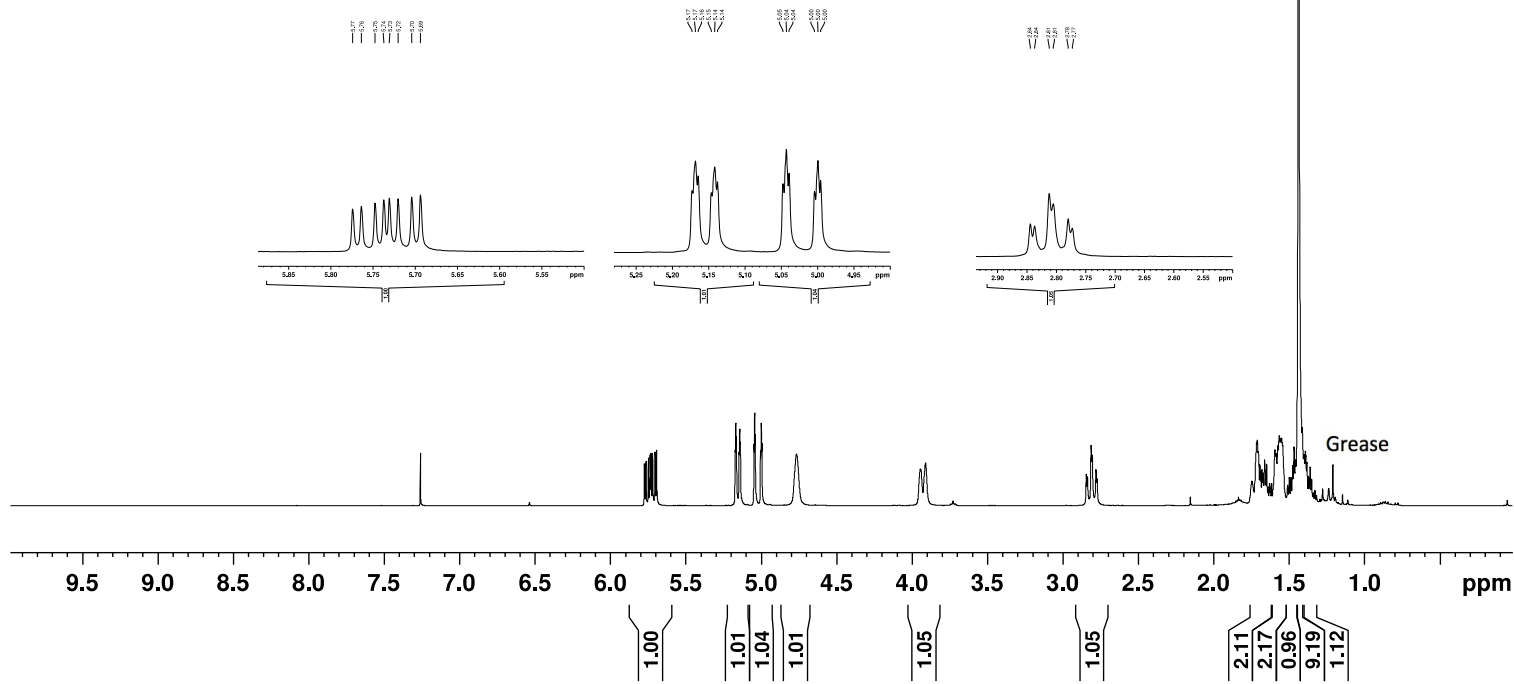

${ }^{13} \mathrm{C}$ NMR $\left(101 \mathrm{MHz}, \mathrm{CDCl}_{3}\right)$

|

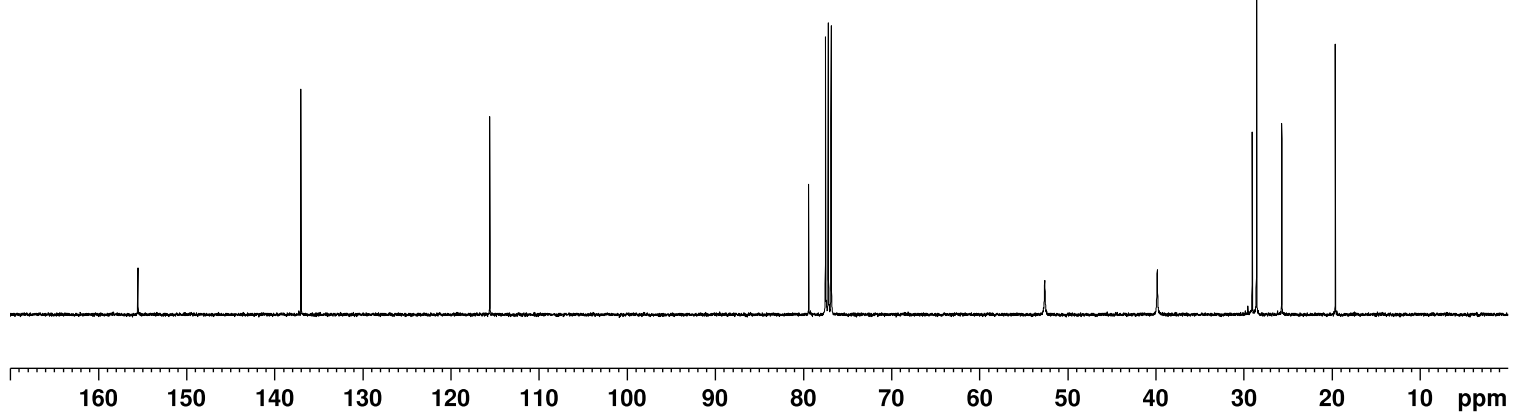


( \pm )-N-Benzyl-cyclohex-2-ene-1-methanamine, 1f' ${ }^{1} \mathrm{H} \mathrm{NMR}\left(400 \mathrm{MHz}, \mathrm{CDCl}_{3}\right)$
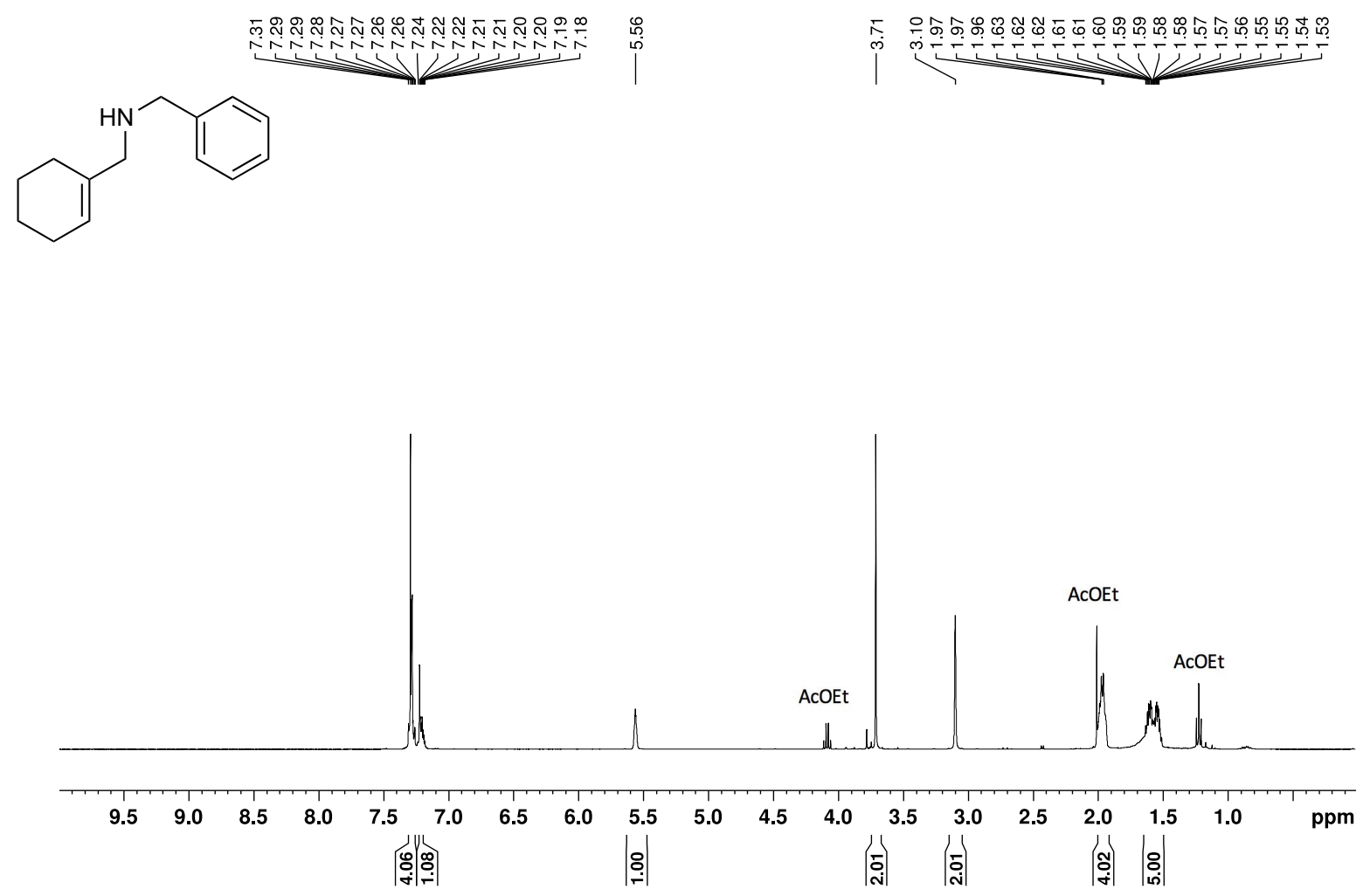

${ }^{13} \mathrm{C}$ NMR (101 MHz, $\left.\mathrm{CDCl}_{3}\right)$

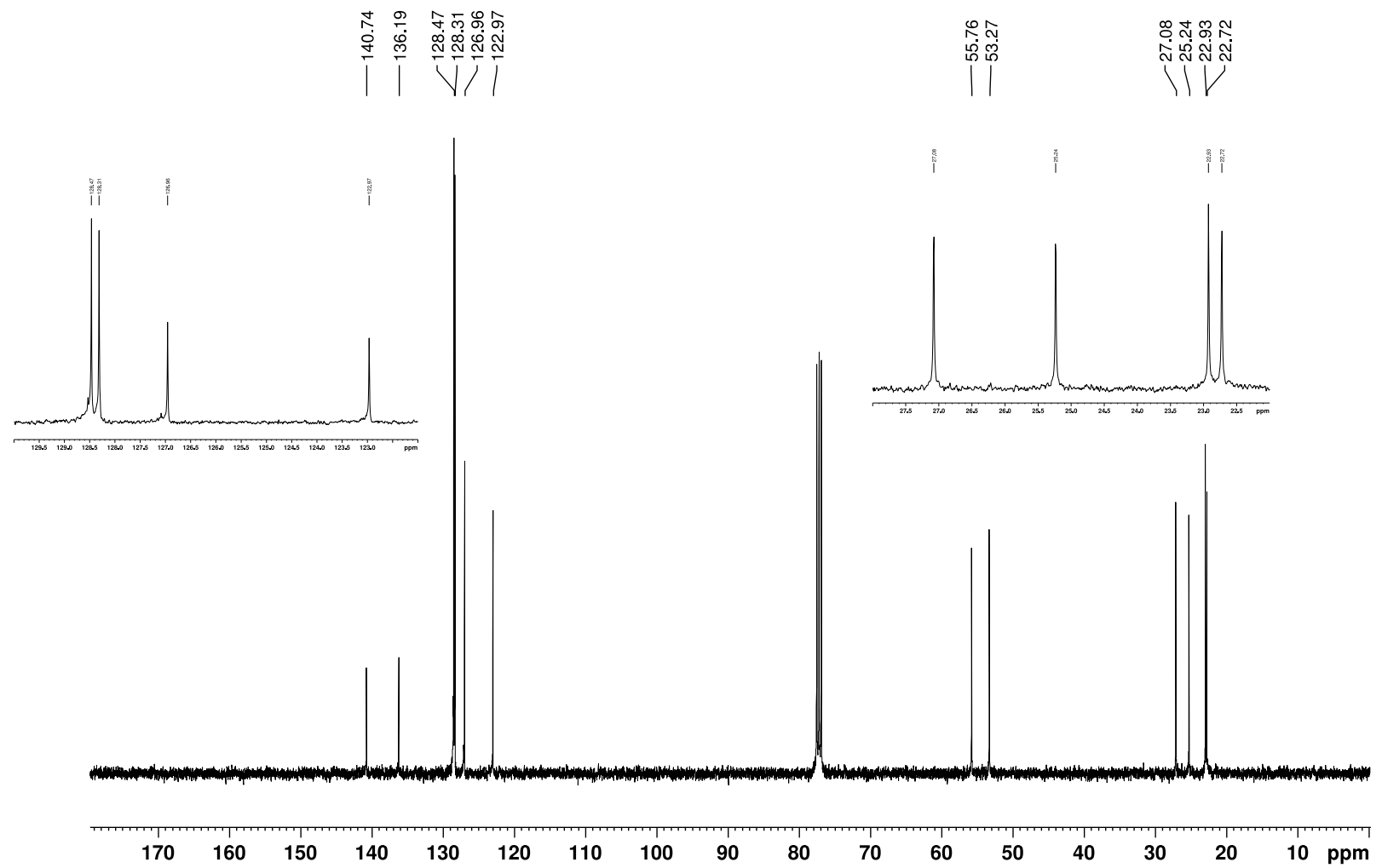


( \pm )- $\mathrm{N}$-Boc, $\mathrm{N}$-Benzyl-cyclohex-2-ene-1-methanamine, $1 \mathrm{f}$ ${ }^{1} \mathrm{H} \mathrm{NMR}\left(400 \mathrm{MHz}, \mathrm{CDCl}_{3}\right)$
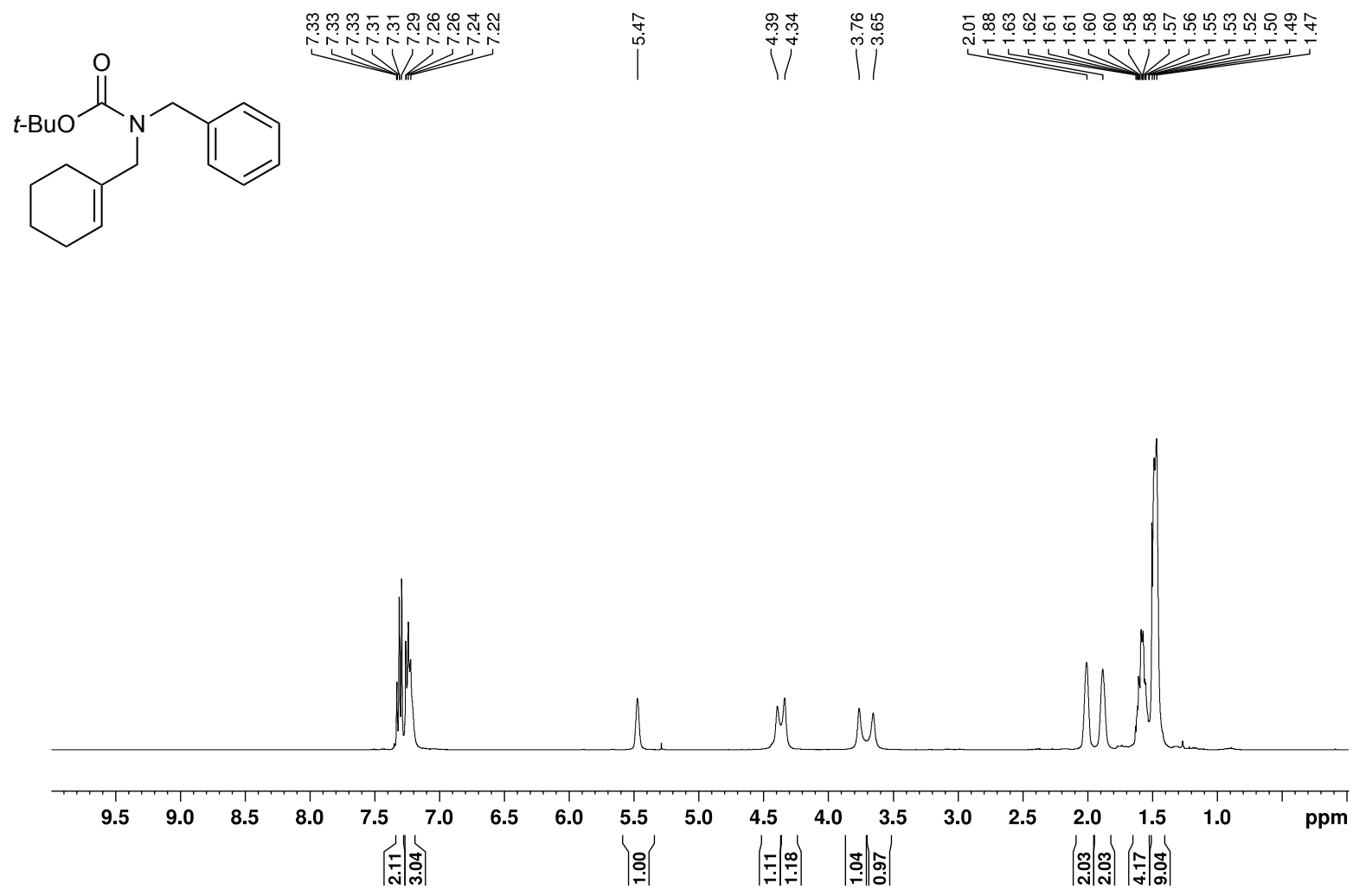

${ }^{13} \mathrm{C}$ NMR $\left(101 \mathrm{MHz}, \mathrm{CDCl}_{3}\right)$

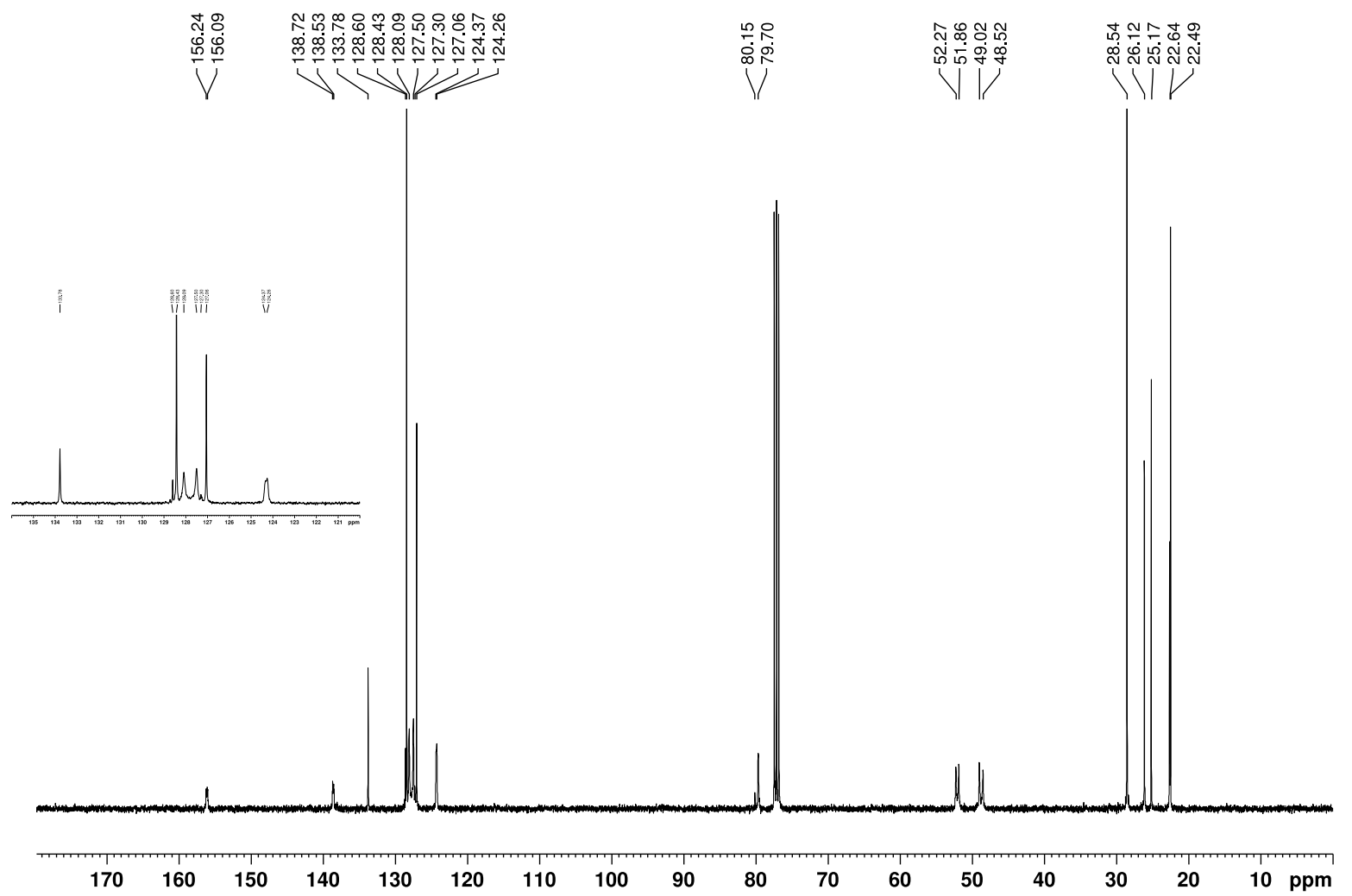


( \pm )-N-Benzyl-5-benzyl-oxazolidin-2-one, 3a ${ }^{1} \mathrm{H} \mathrm{NMR}\left(400 \mathrm{MHz}, \mathrm{CDCl}_{3}\right)$

ஜ

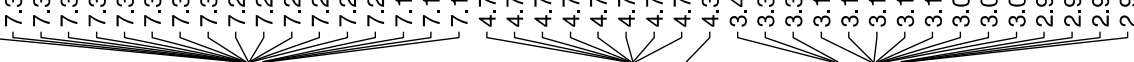<smiles>O=C1OC(Cc2ccccc2)CN1Cc1ccccc1</smiles>

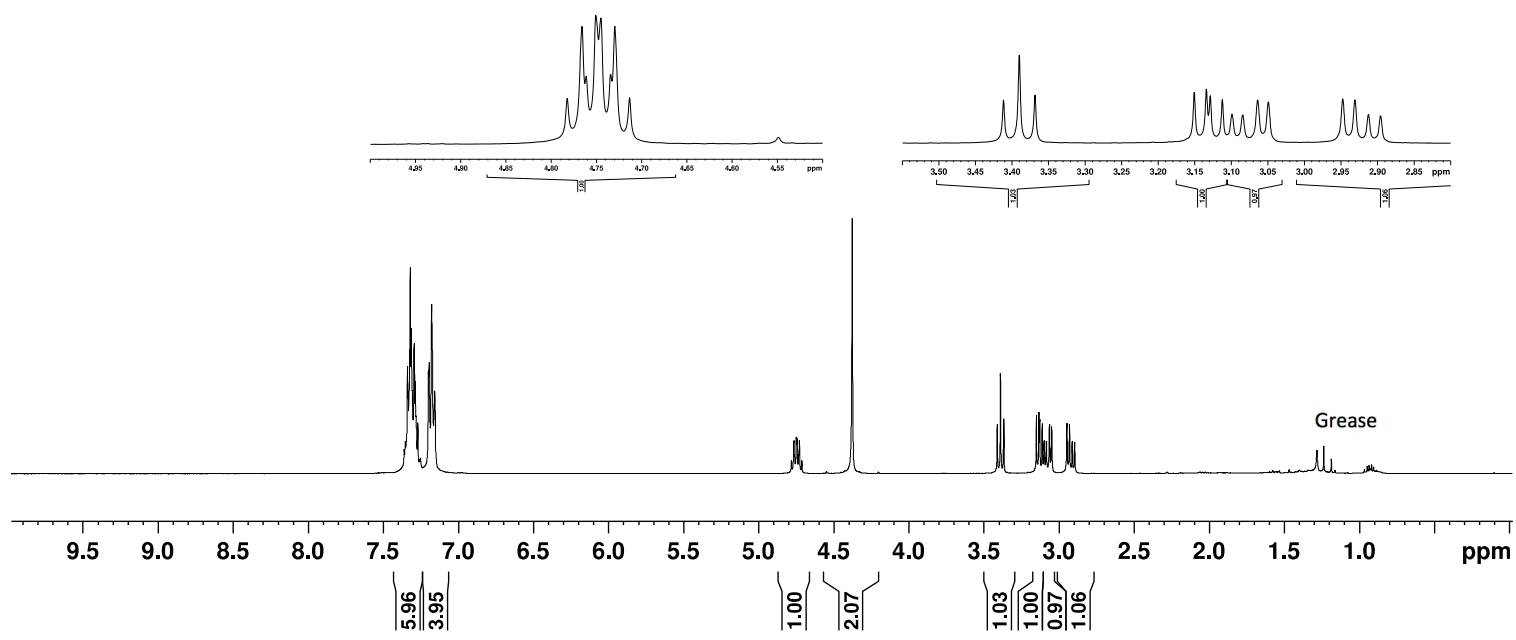

${ }^{13} \mathrm{C}$ NMR $\left(101 \mathrm{MHz}, \mathrm{CDCl}_{3}\right)$
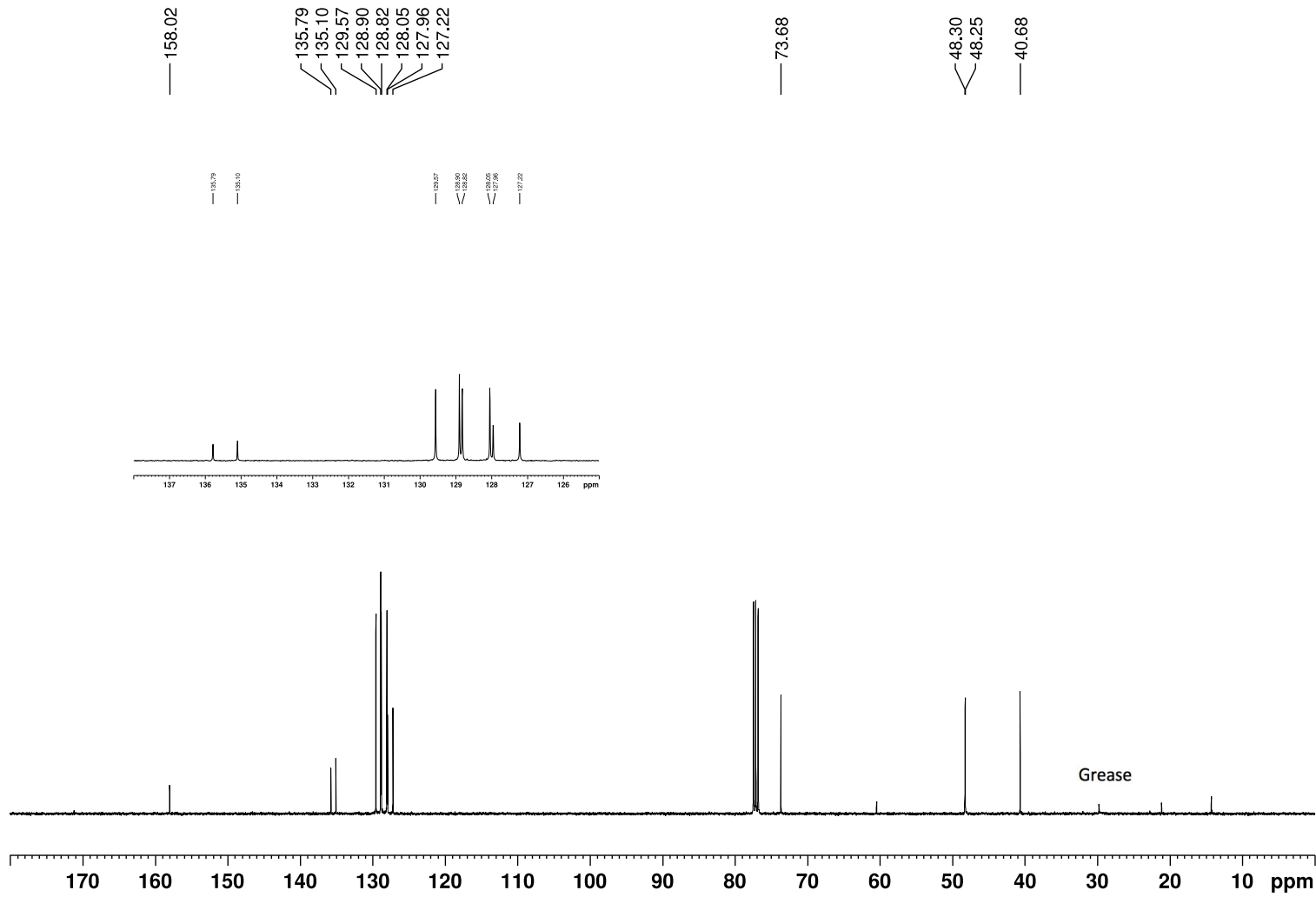
( \pm )-N-Benzyl-5-(p-fluorobenzyl)-oxazolidin-2-one, 3b

${ }^{1} \mathrm{H} \mathrm{NMR}\left(400 \mathrm{MHz}, \mathrm{CDCl}_{3}\right)$

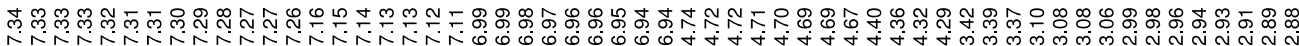<smiles>O=C1OC(Cc2ccc(F)cc2)CN1Cc1ccccc1</smiles>

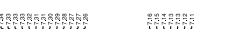

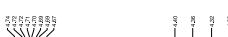

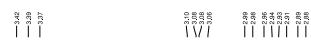
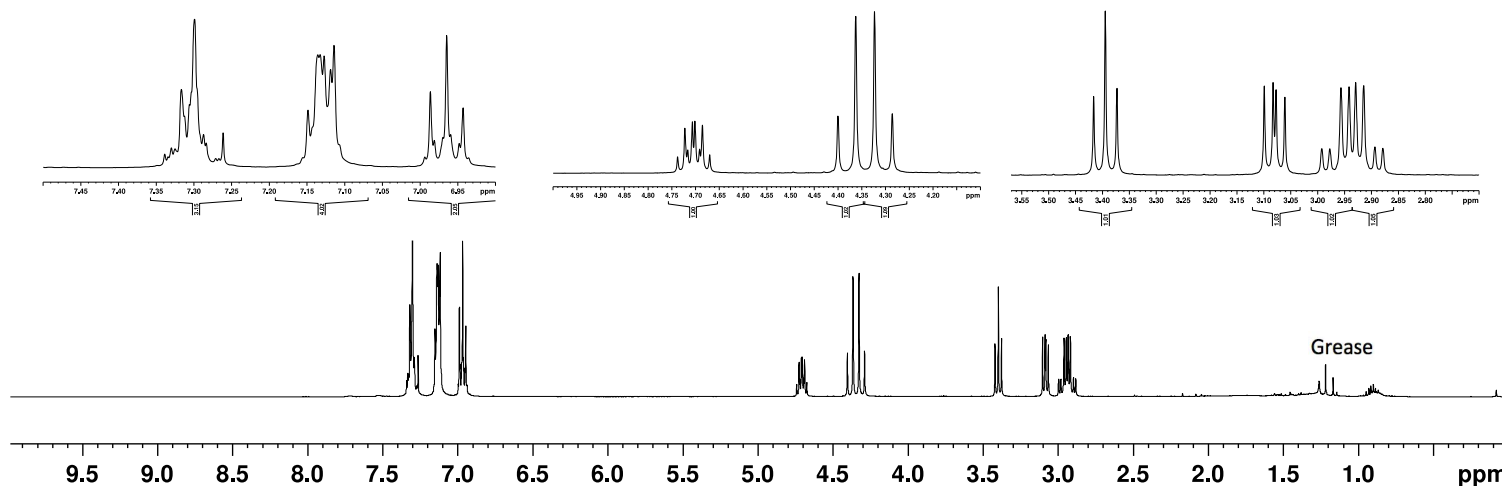

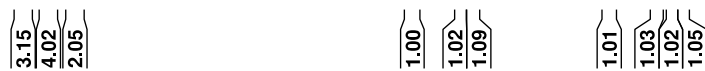

${ }^{13} \mathrm{C} \mathrm{NMR}\left(101 \mathrm{MHz}, \mathrm{CDCl}_{3}\right)$
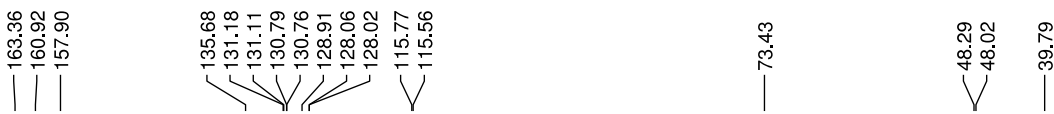

帮籍
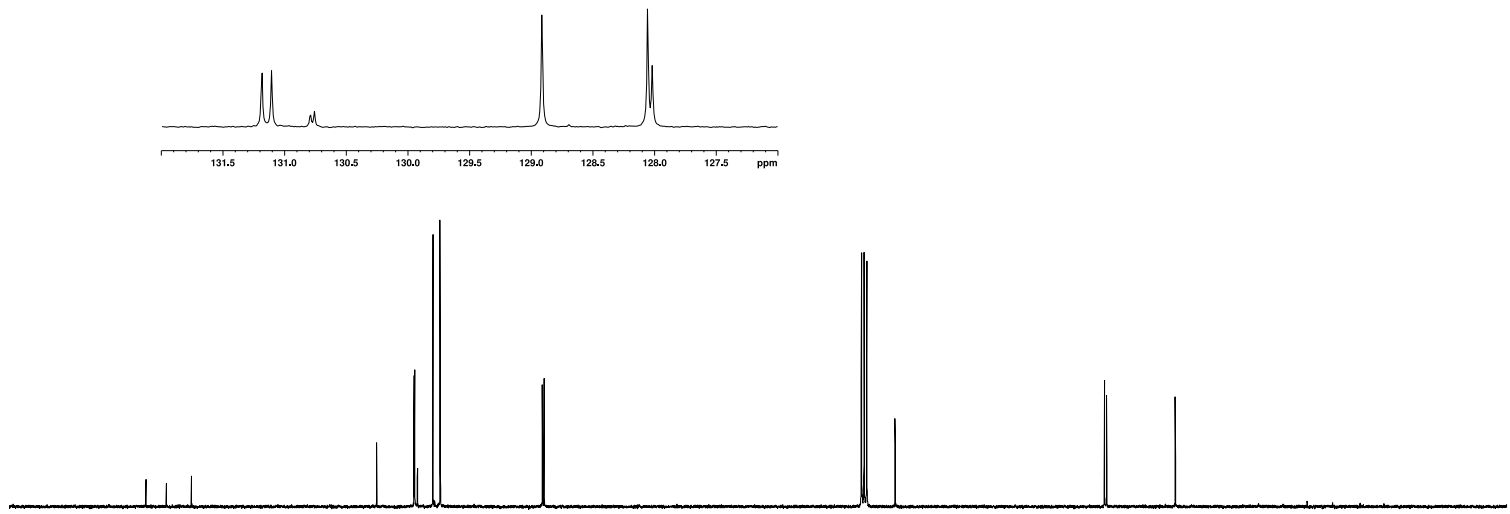

$\begin{array}{lllllllllllllllll}170 & 160 & 150 & 140 & 130 & 120 & 110 & 100 & 90 & 80 & 70 & 60 & 50 & 40 & 30 & 20 & 10\end{array}$ 
${ }^{19} \mathrm{~F}$ NMR (376 $\left.\mathrm{MHz}, \mathrm{CDCl}_{3}\right)$

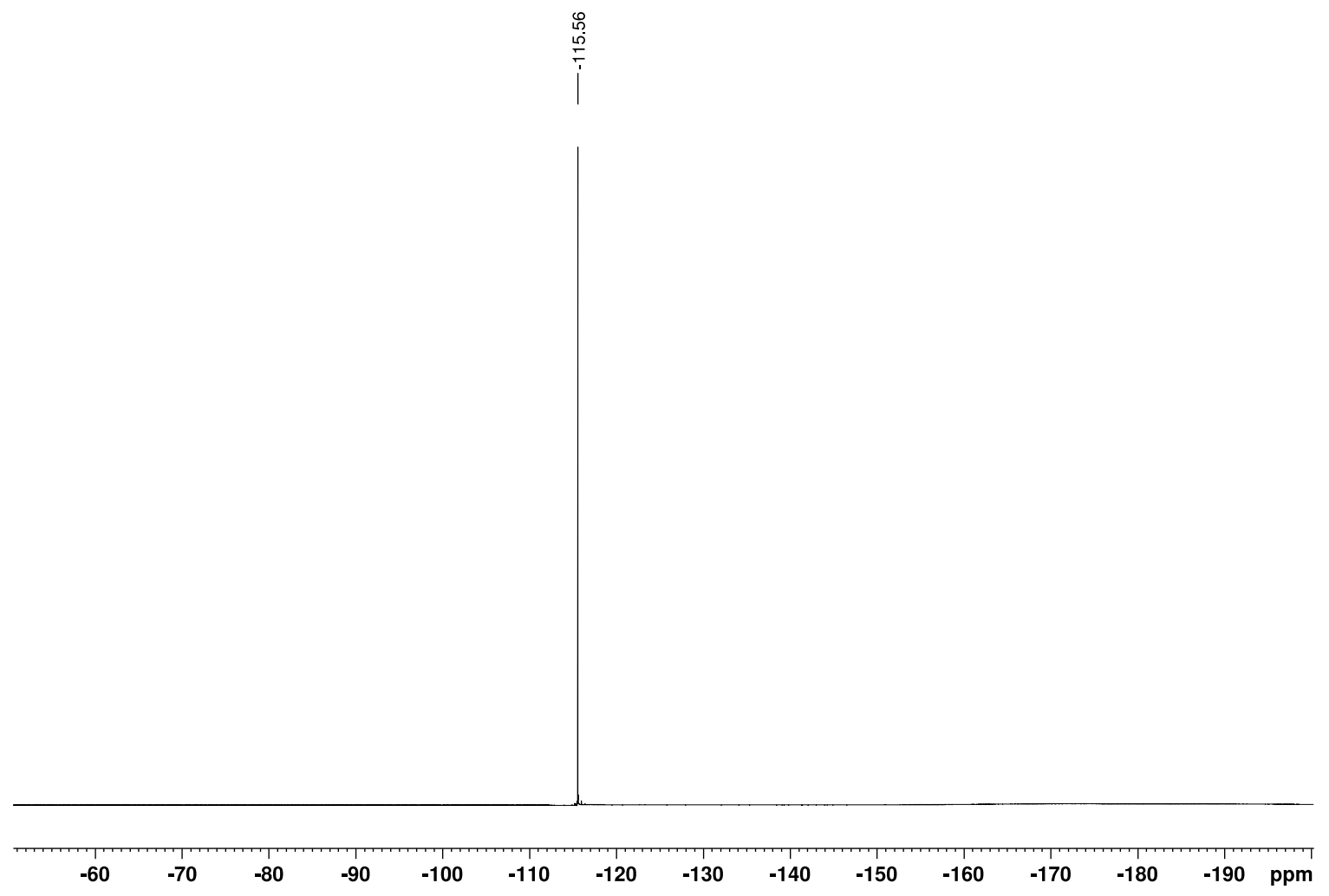


( \pm )- $N$-Benzyl-5-(p-methylbenzyl)-oxazolidin-2-one, 3c

${ }^{1} \mathrm{H}$ NMR $\left(400 \mathrm{MHz}, \mathrm{CDCl}_{3}\right)$

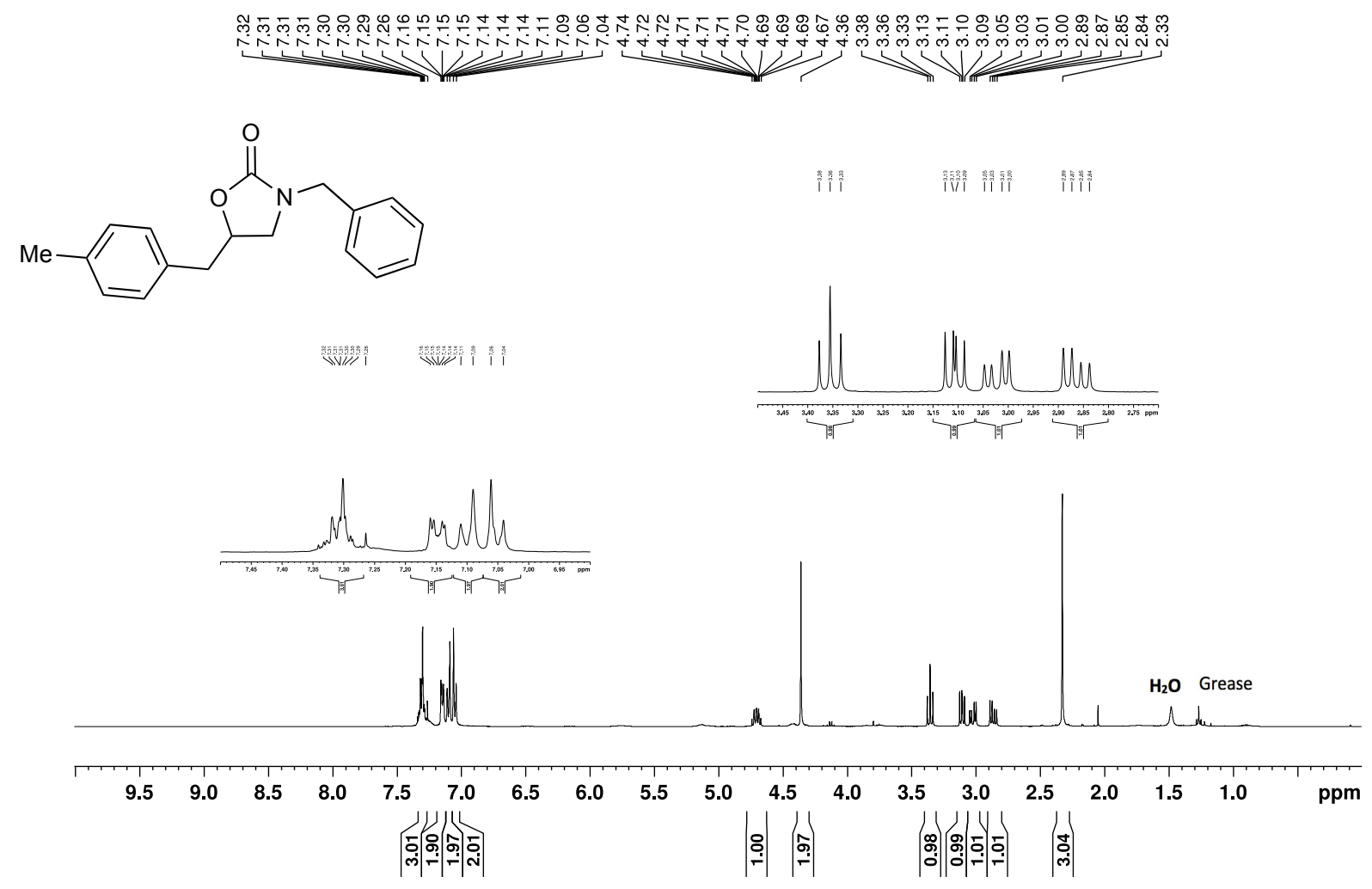

${ }^{13} \mathrm{C}$ NMR $\left(101 \mathrm{MHz}, \mathrm{CDCl}_{3}\right)$
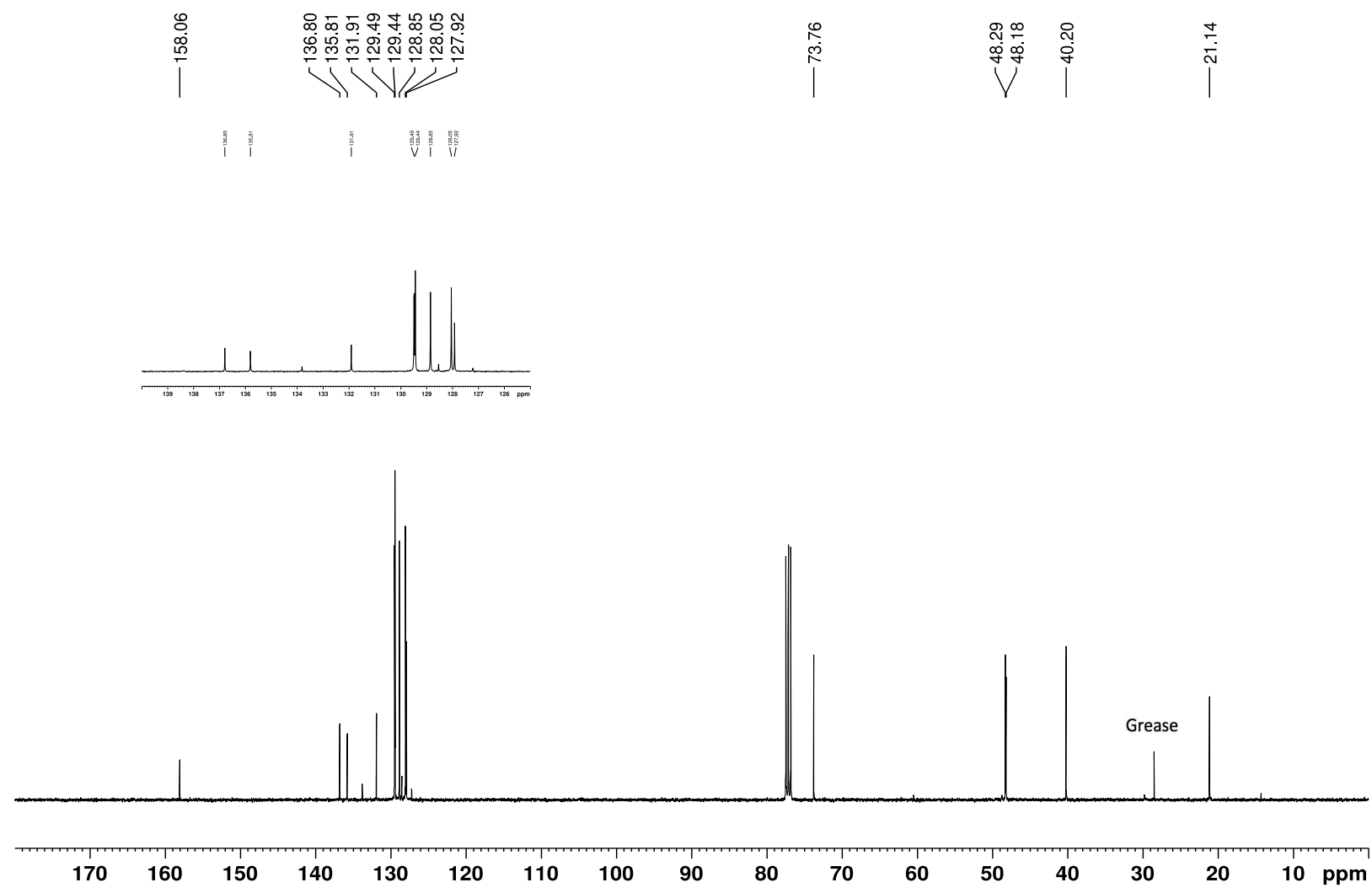
( \pm )- $N$-Benzyl-5-(p-methoxybenzyl)-oxazolidin-2-one, 3d

${ }^{1} \mathrm{H}$ NMR $\left(400 \mathrm{MHz}, \mathrm{CDCl}_{3}\right)$

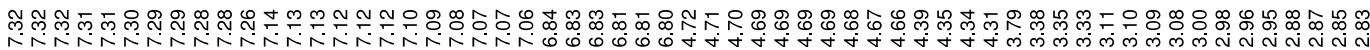<smiles>COc1ccc(CC2CN(Cc3ccccc3)C(=O)O2)cc1</smiles>

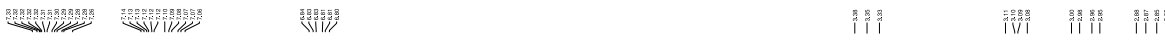
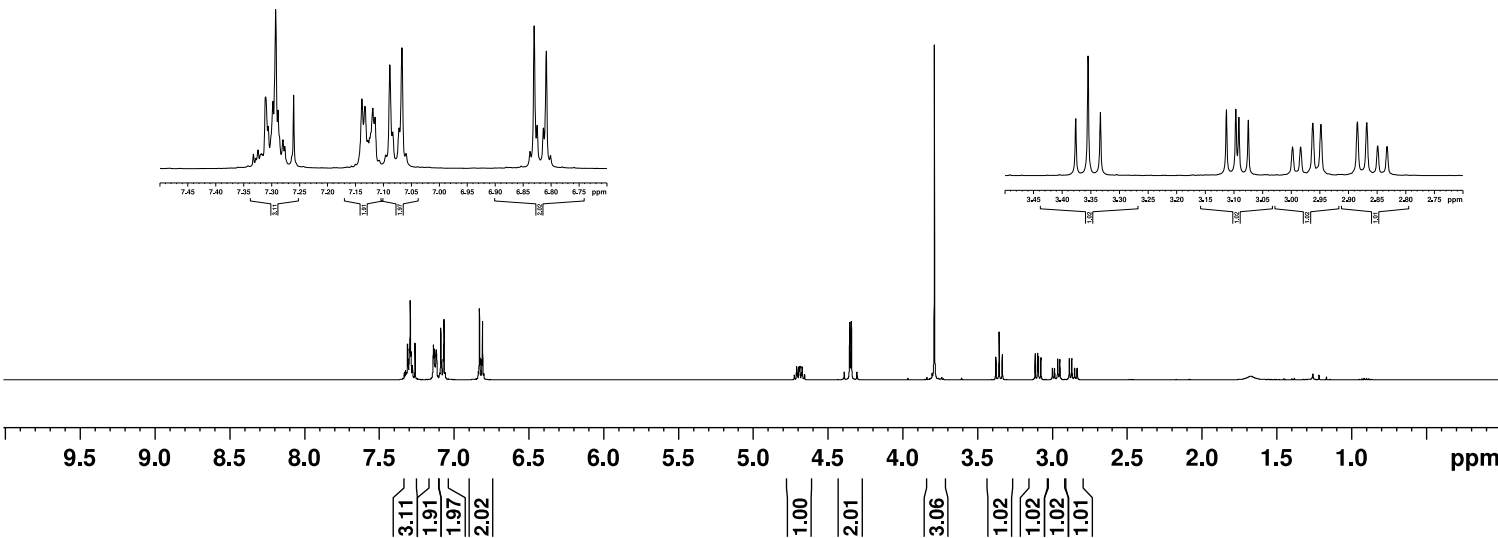

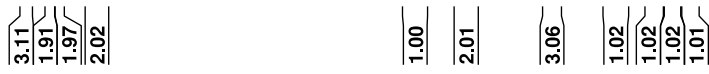

${ }^{13} \mathrm{C}$ NMR $\left(101 \mathrm{MHz}, \mathrm{CDCl}_{3}\right)$

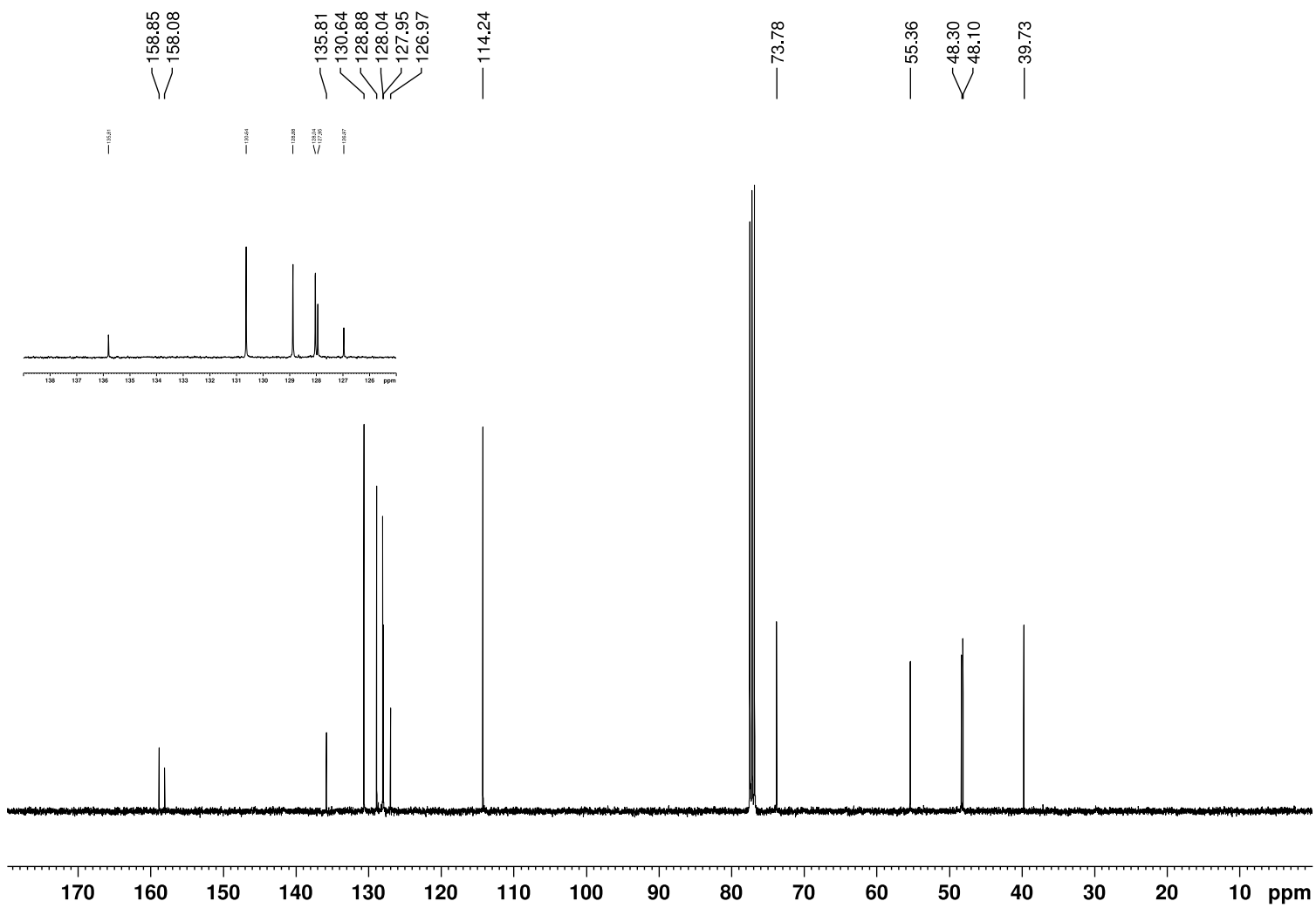


( \pm )-N-Benzyl-5-(m-methoxybenzyl)-oxazolidin-2-one, 3e ${ }^{1} \mathrm{H}$ NMR (400 MHz, $\mathrm{CDCl}_{3}$ )

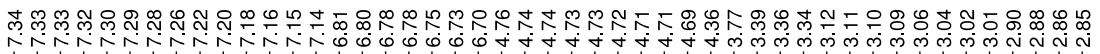<smiles>COc1cccc(CC2CN(Cc3ccccc3)C(=O)O2)c1</smiles>

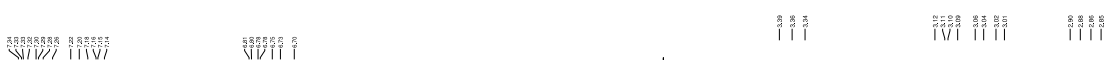

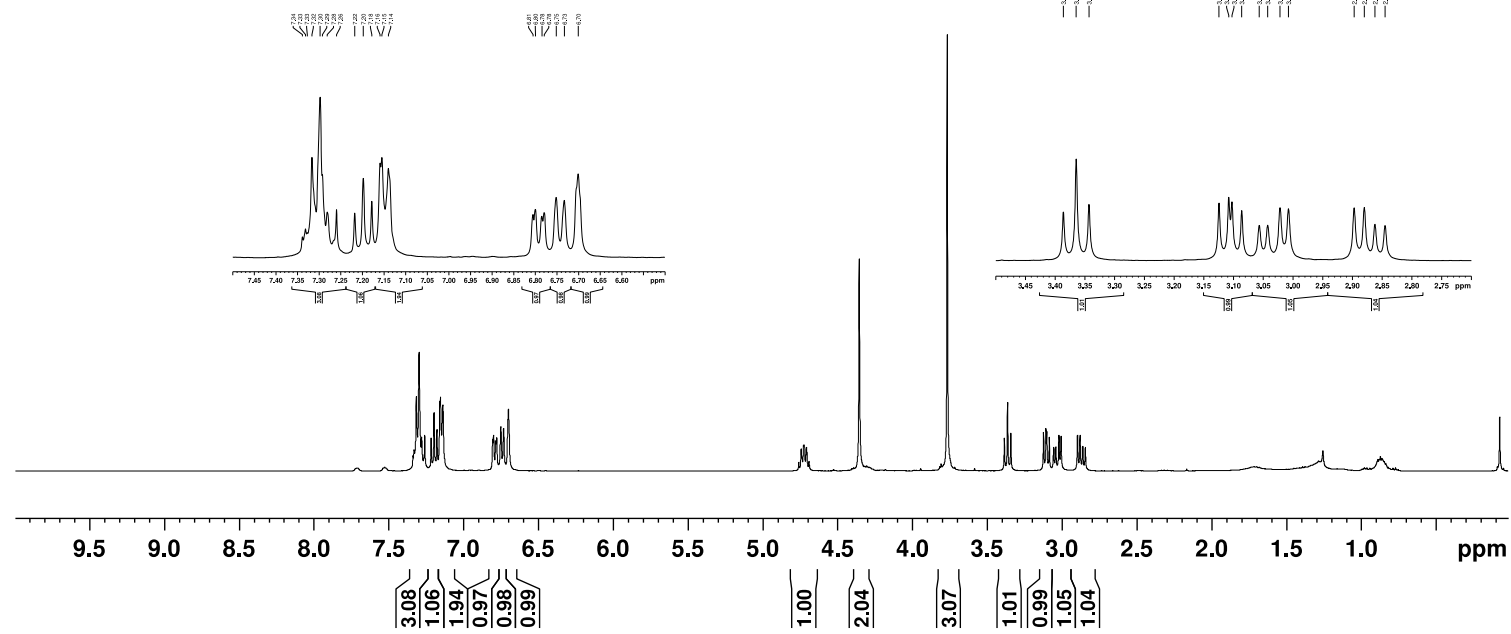

${ }^{13} \mathrm{C} \mathrm{NMR}\left(101 \mathrm{MHz}, \mathrm{CDCl}_{3}\right)$
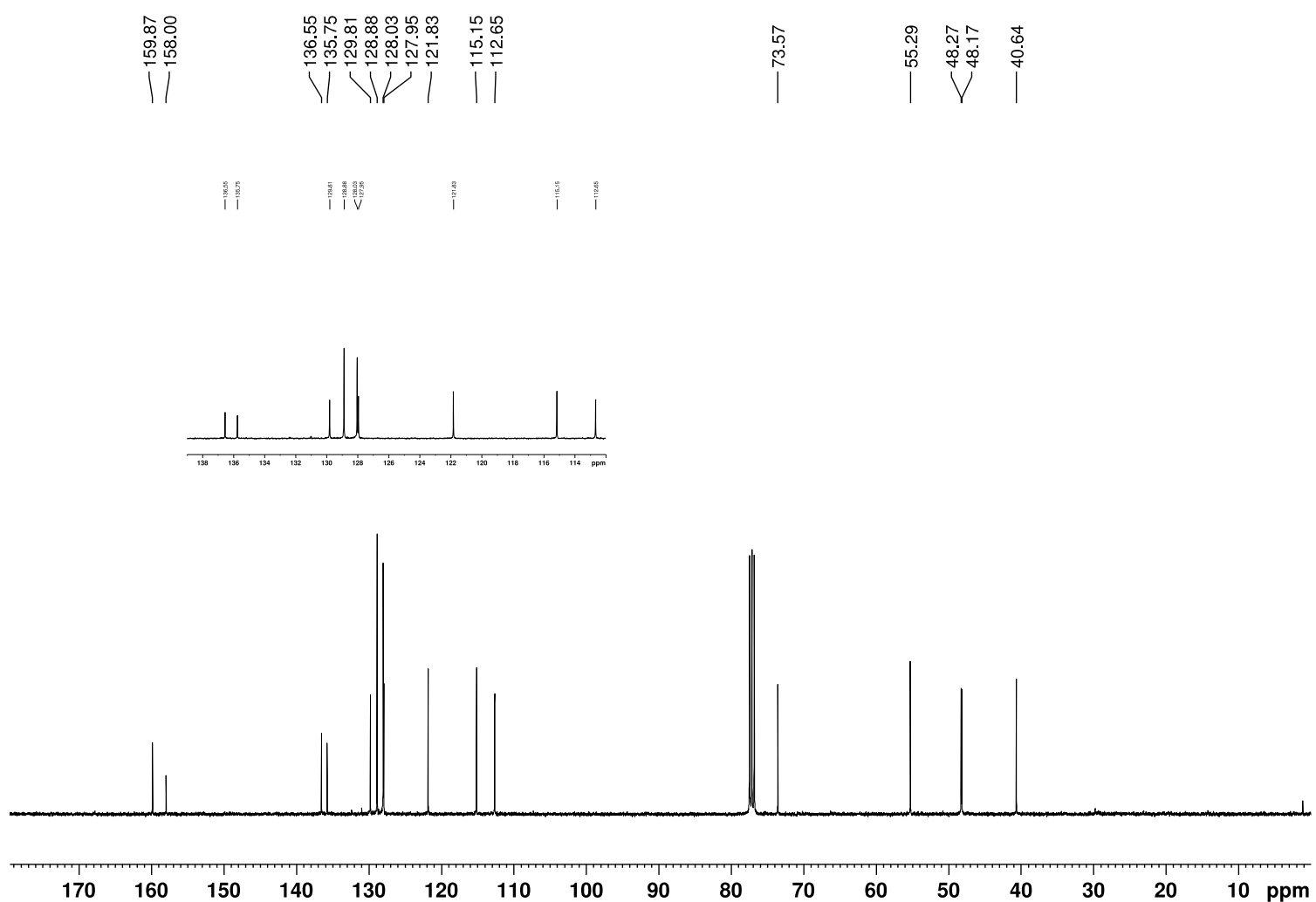
( \pm )-N-Benzyl-5-(o-methylbenzyl)-oxazolidin-2-one, $3 f$

${ }^{1} \mathrm{H} \mathrm{NMR}\left(400 \mathrm{MHz}, \mathrm{CDCl}_{3}\right)$

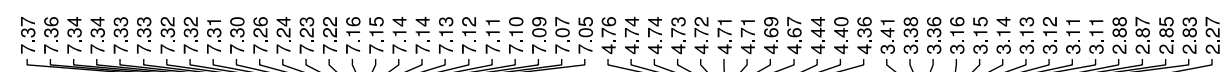

(1)

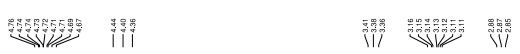
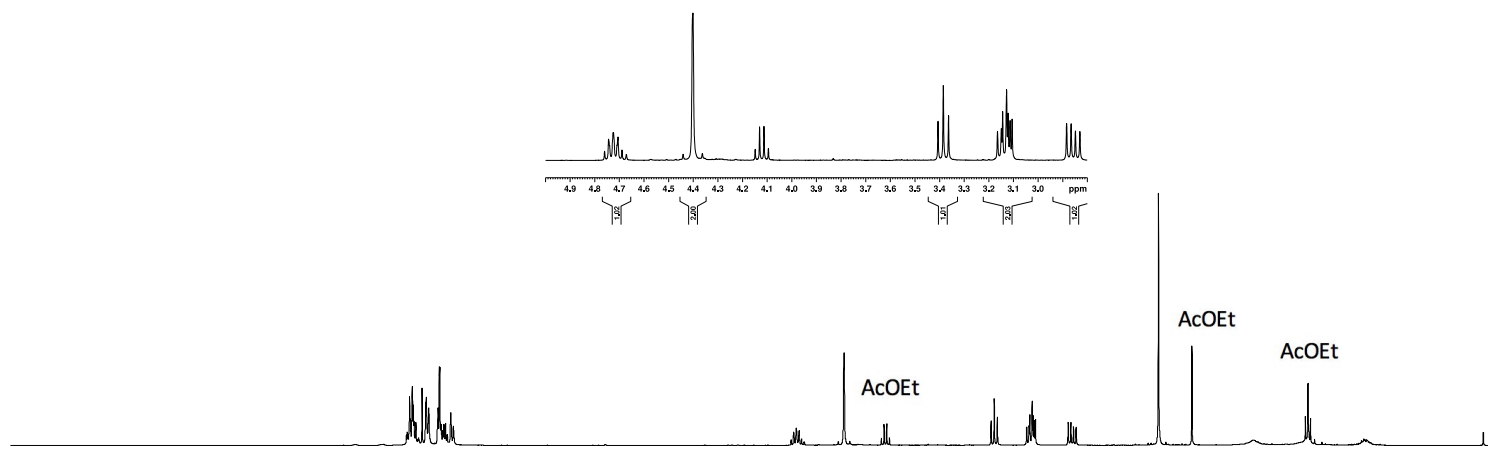

$\begin{array}{lllllllllllllllllllll}9.5 & 9.0 & 8.5 & 8.0 & 7.5 & 7.0 & 6.5 & 6.0 & 5.5 & 5.0 & 4.5 & 4.0 & 3.5 & 3.0 & 2.5 & 2.0 & 1.5 & 1.0 & \mathrm{ppm}\end{array}$

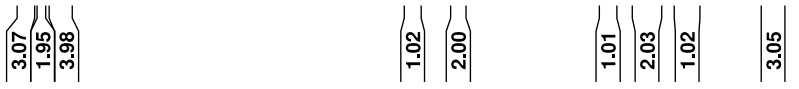

${ }^{13} \mathrm{C}$ NMR $\left(101 \mathrm{MHz}, \mathrm{CDCl}_{3}\right)$

$\stackrel{8}{8} \quad$ \&

要 $\quad$

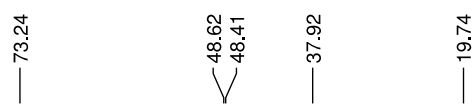
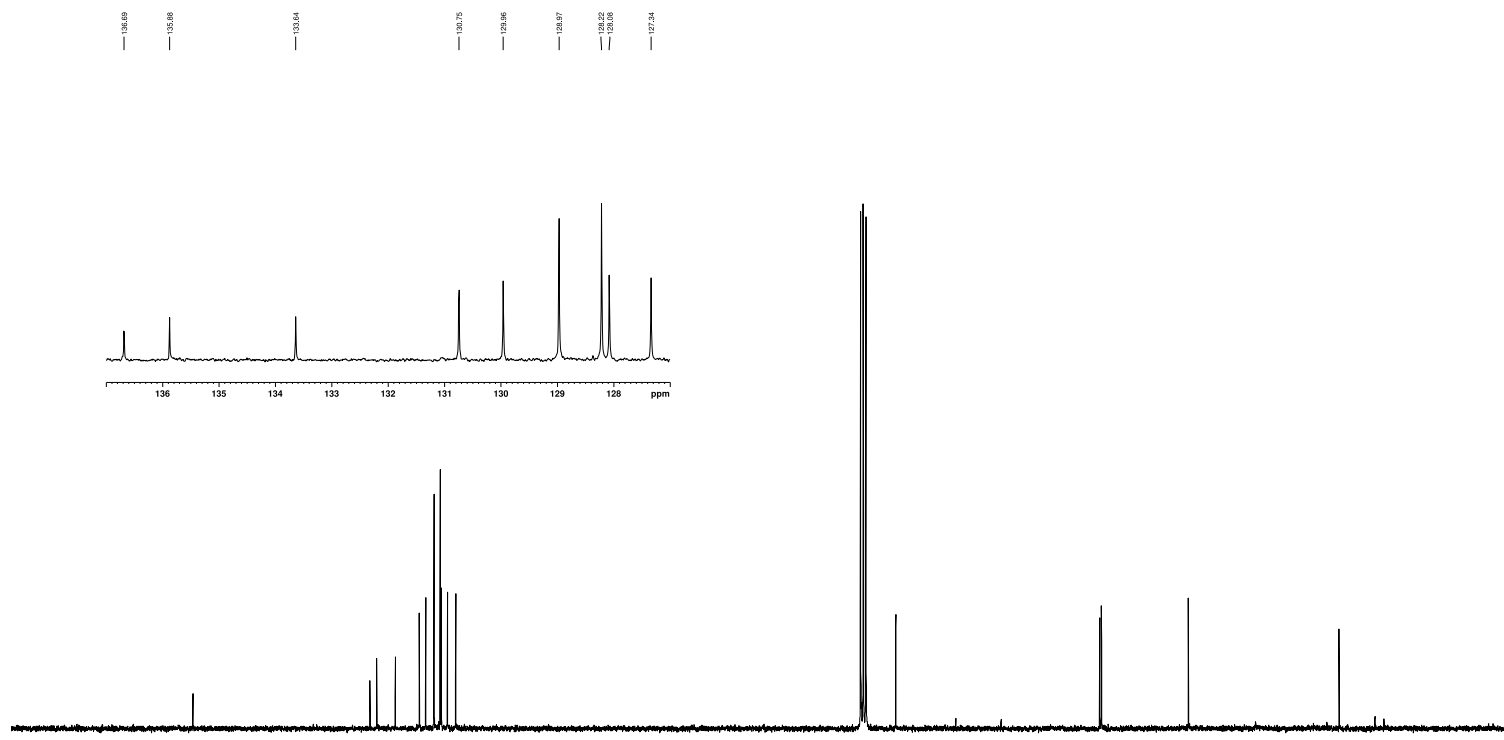

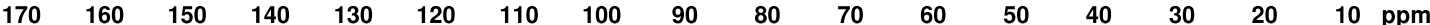


( \pm )-N-Benzyl-5-(o-methoxybenzyl)-oxazolidin-2-one, 3g ${ }^{1} \mathrm{H}$ NMR $\left(400 \mathrm{MHz}, \mathrm{CDCl}_{3}\right)$

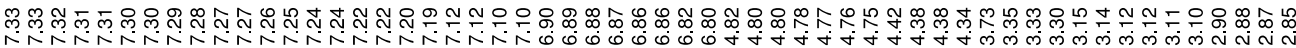<smiles>COc1ccccc1CC1CN(Cc2ccccc2)C(=O)O1</smiles>

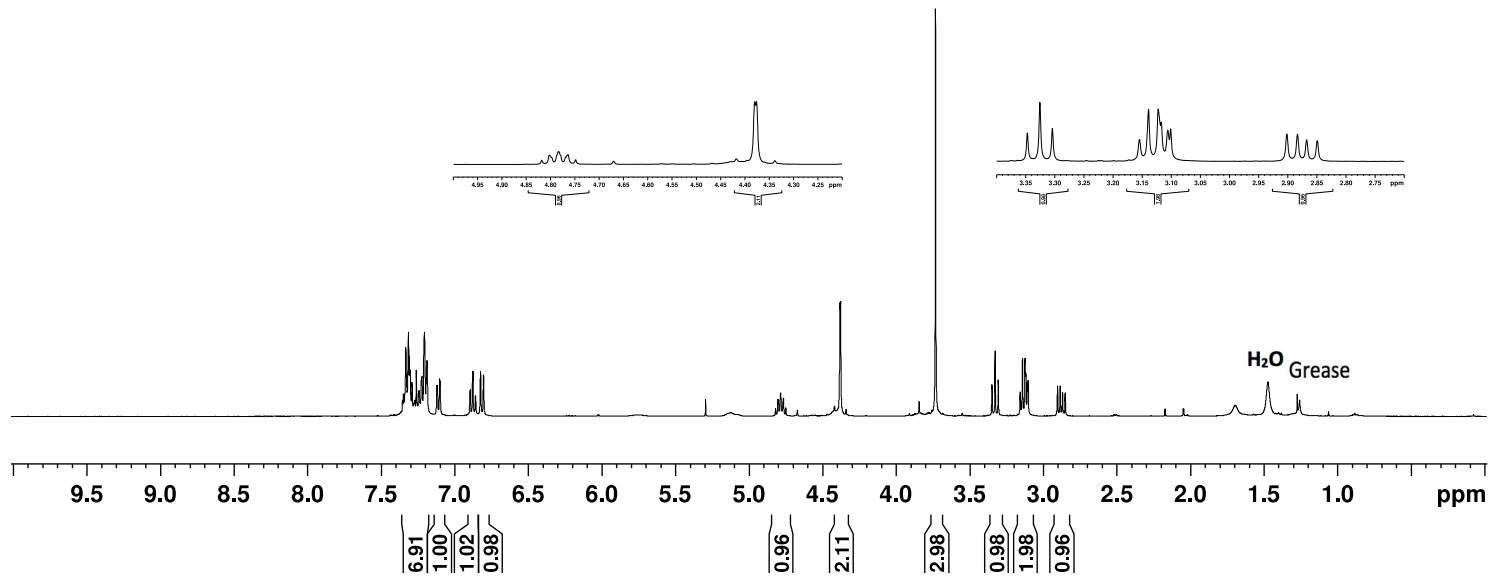

${ }^{13} \mathrm{C}$ NMR $\left(101 \mathrm{MHz}, \mathrm{CDCl}_{3}\right)$

$\sum^{\mid}$
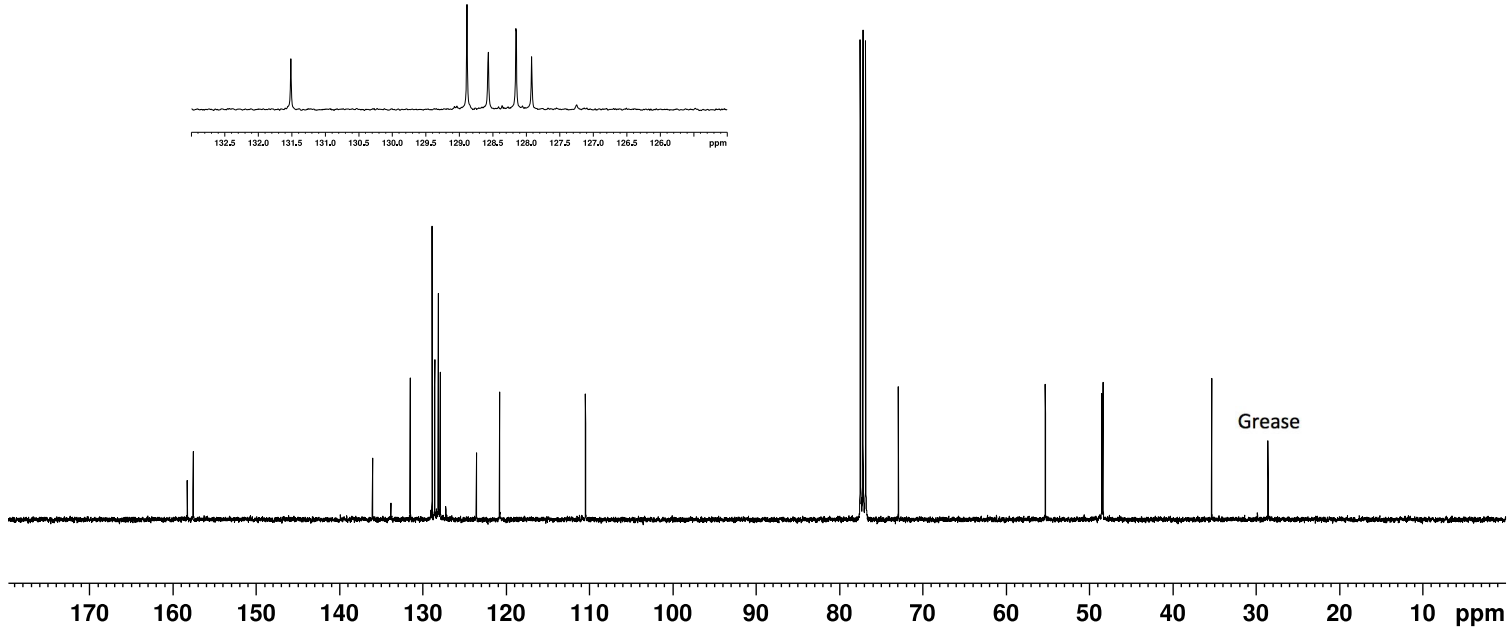
(-)-[(R)- $N$-Phenylethyl]-(5S)-benzyl-oxazolidin-2-one, 3h

${ }^{1} \mathrm{H} \mathrm{NMR}\left(400 \mathrm{MHz}, \mathrm{CDCl}_{3}\right)$

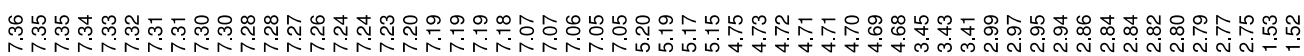
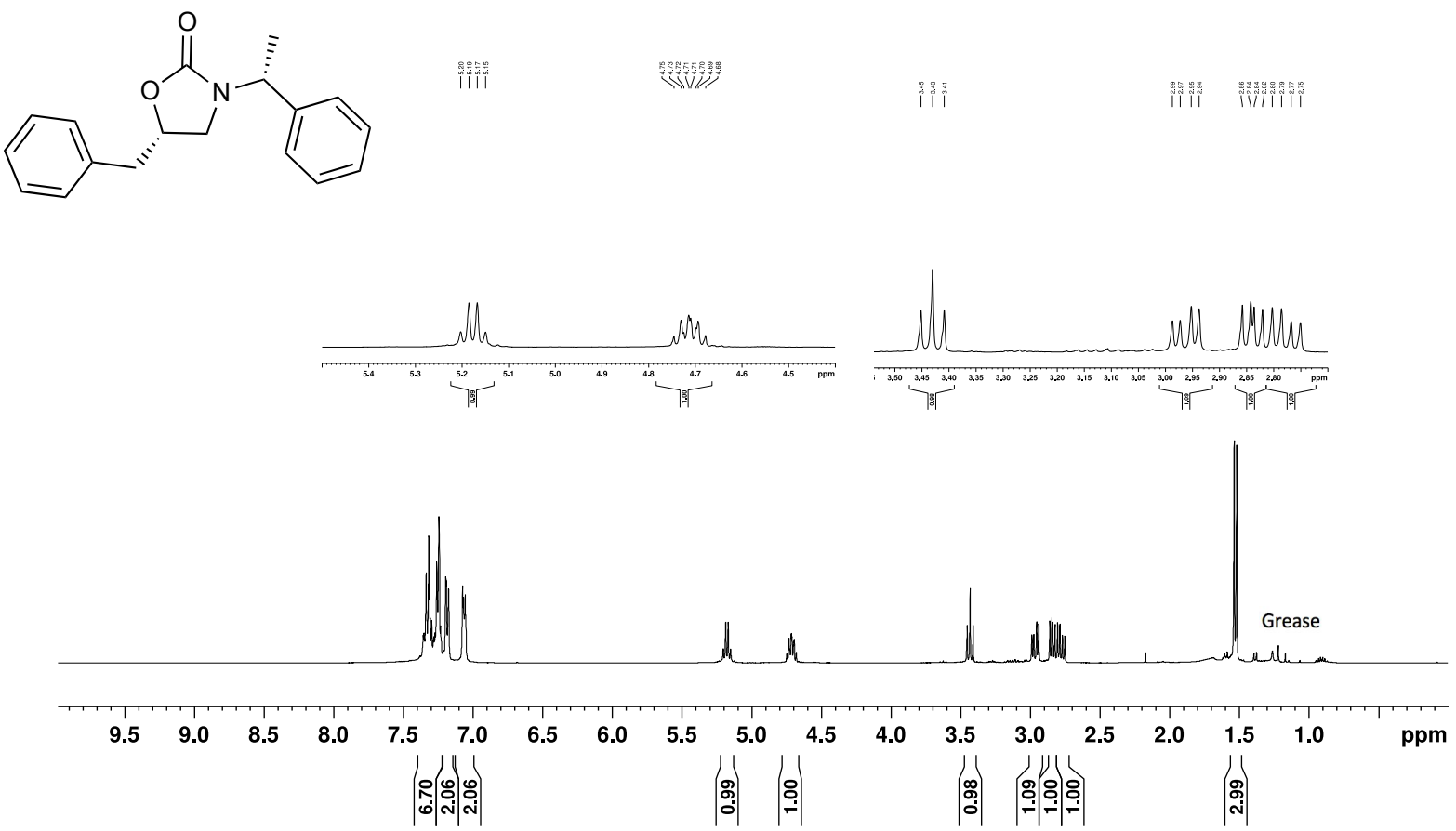

${ }^{13} \mathrm{C}$ NMR $\left(101 \mathrm{MHz}, \mathrm{CDCl}_{3}\right)$
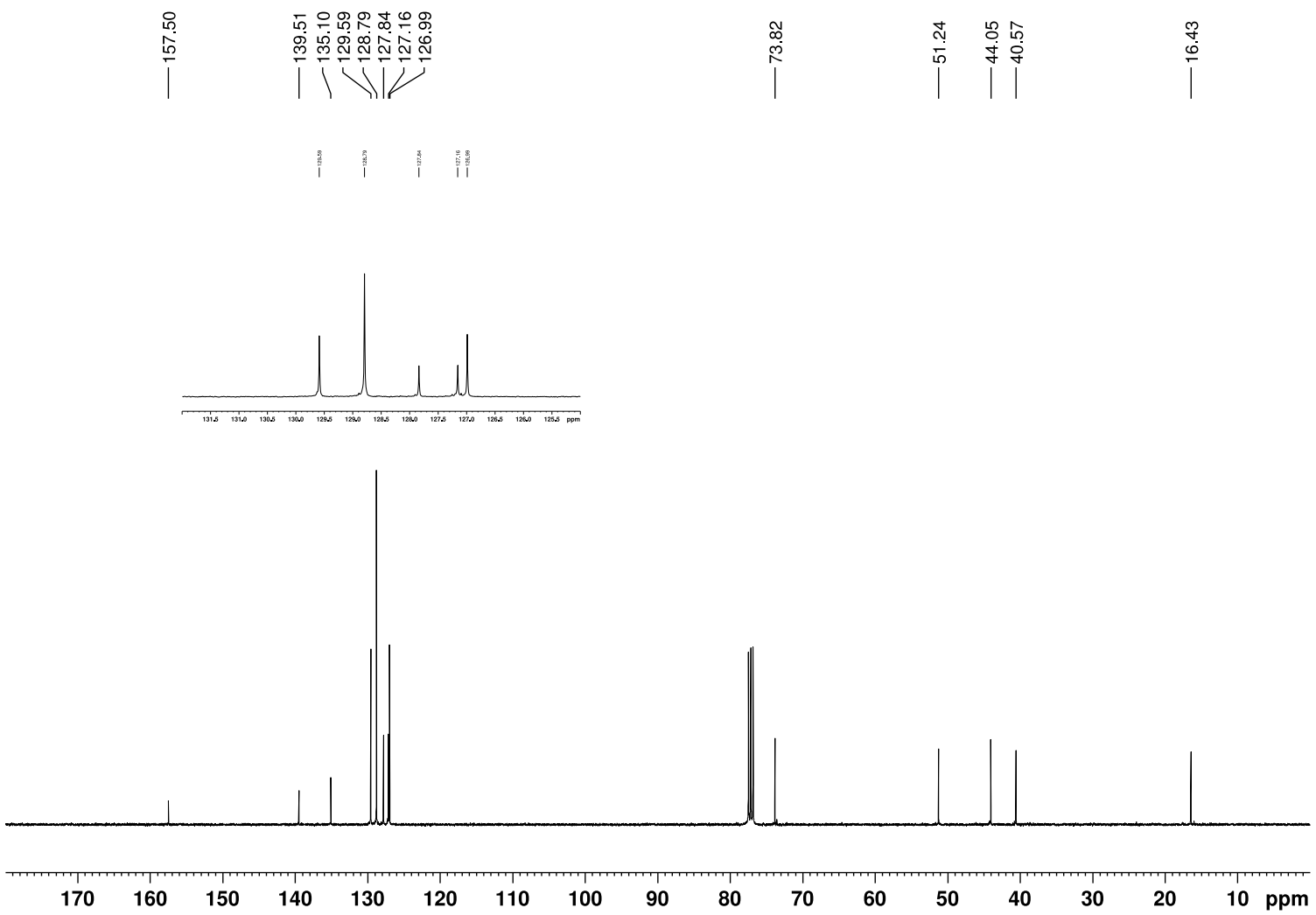
(+)-[(R)-N-Phenylethyl]-(5R)-benzyl-oxazolidin-2-one, 3i

${ }^{1} \mathrm{H} \mathrm{NMR}\left(400 \mathrm{MHz}, \mathrm{CDCl}_{3}\right)$

管 ـ<smiles>C[C@H](c1ccccc1)N1C[C@H](Cc2ccccc2)OC1=O</smiles>

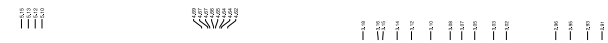

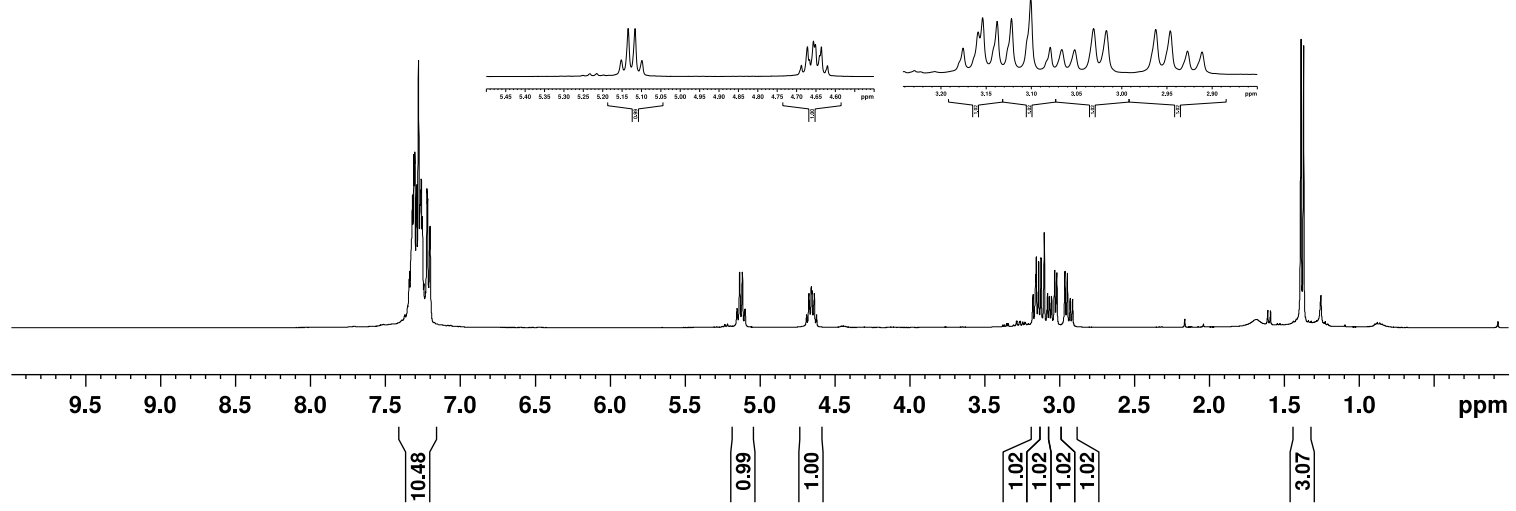

${ }^{13} \mathrm{C}$ NMR $\left(101 \mathrm{MHz}^{\mathrm{CDCl}}{ }_{3}\right)$
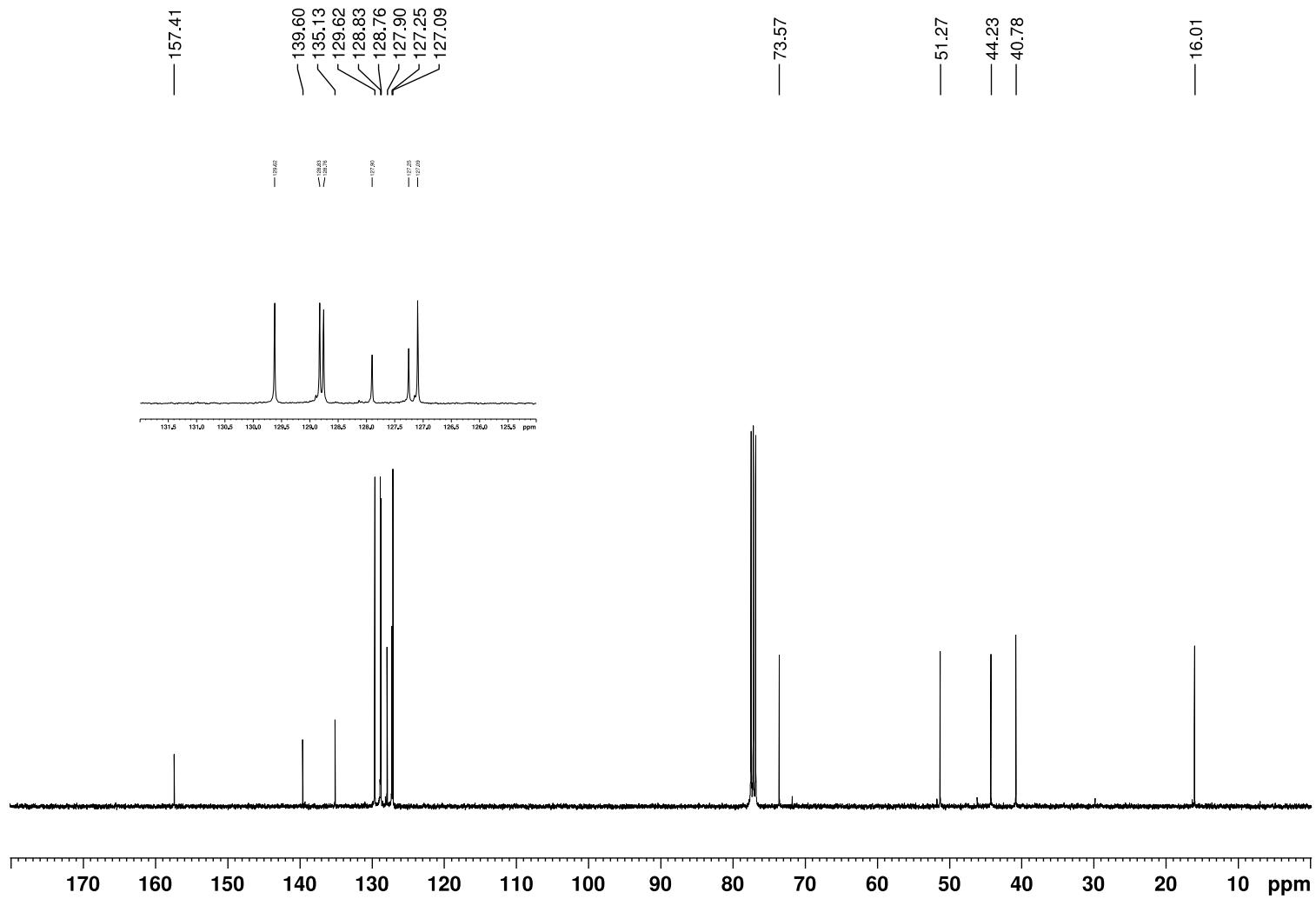
( \pm )-N-Benzyl-7-phenylhexahydrobenzo[d]oxazol-2(3H)-one, 3j

${ }^{1} \mathrm{H}$ NMR $\left(400 \mathrm{MHz}, \mathrm{CDCl}_{3}\right)$

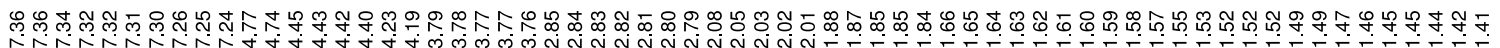<smiles>O=C1O[C@H]2[C@@H](c3ccccc3)CCC[C@H]2N1Cc1ccccc1</smiles>

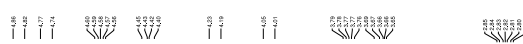

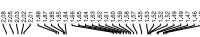
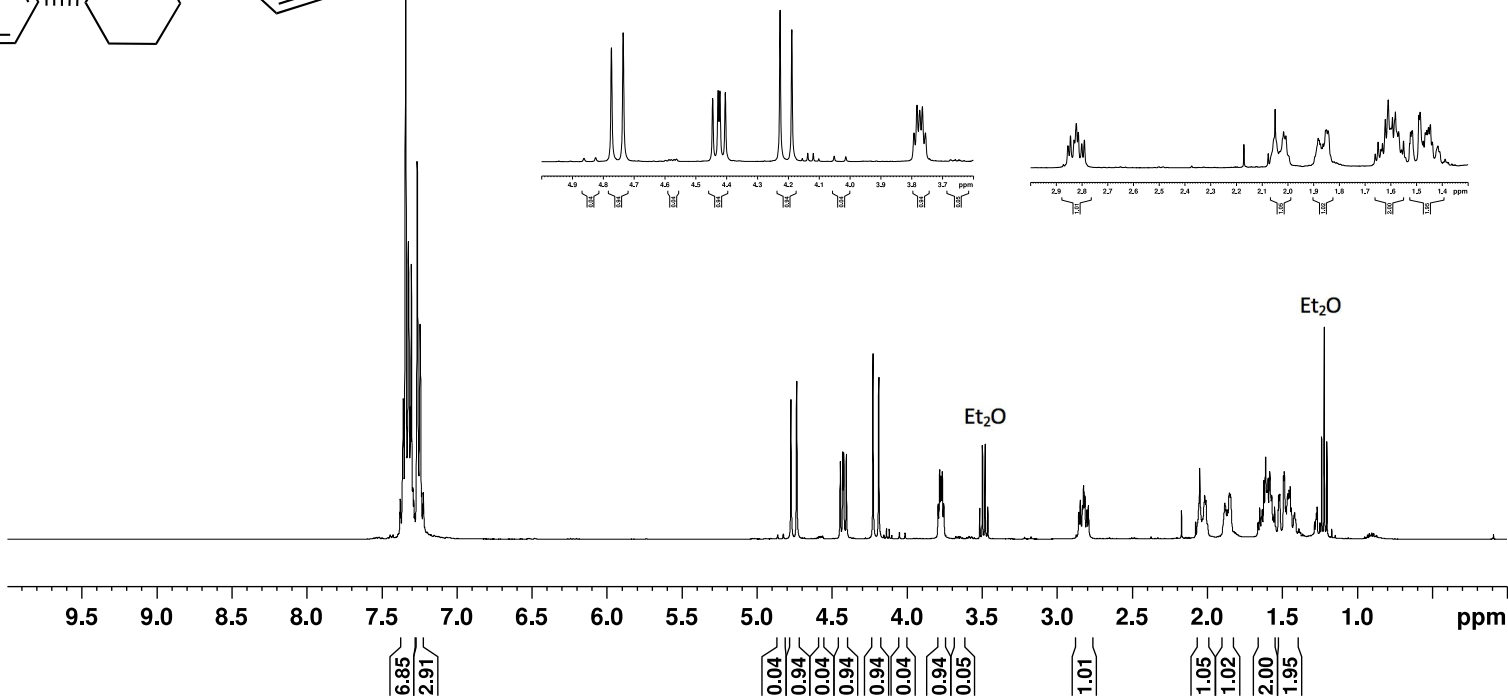

${ }^{13} \mathrm{C}$ NMR $\left(101 \mathrm{MHz}, \mathrm{CDCl}_{3}\right)$

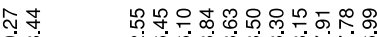

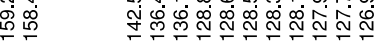

$1+1$

20
0
0

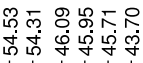

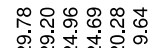

V V V

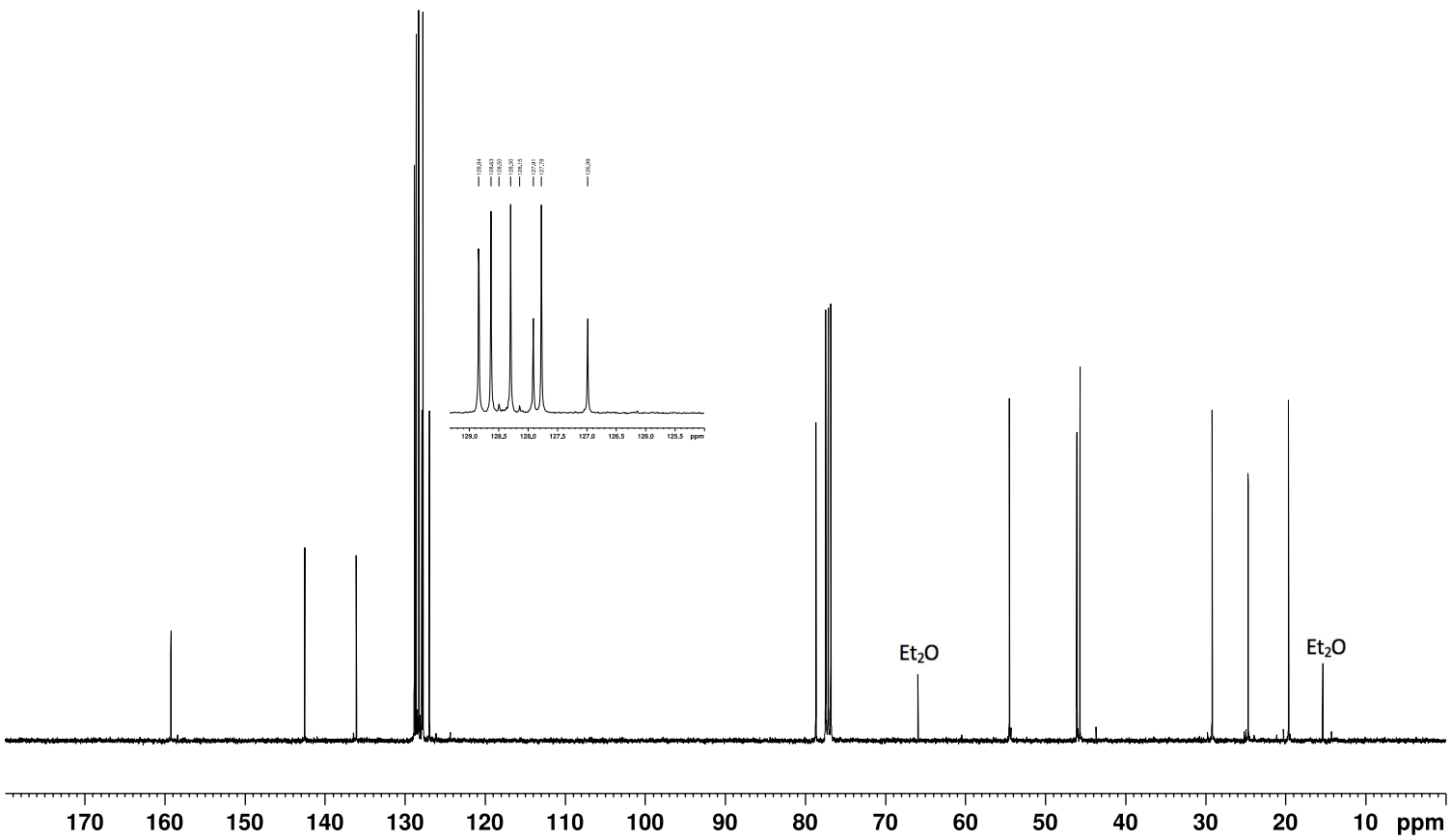


HSQC 2D-NMR (DEPT $135 /{ }^{1} \mathrm{H}, \mathrm{CDCl}_{3}$ )

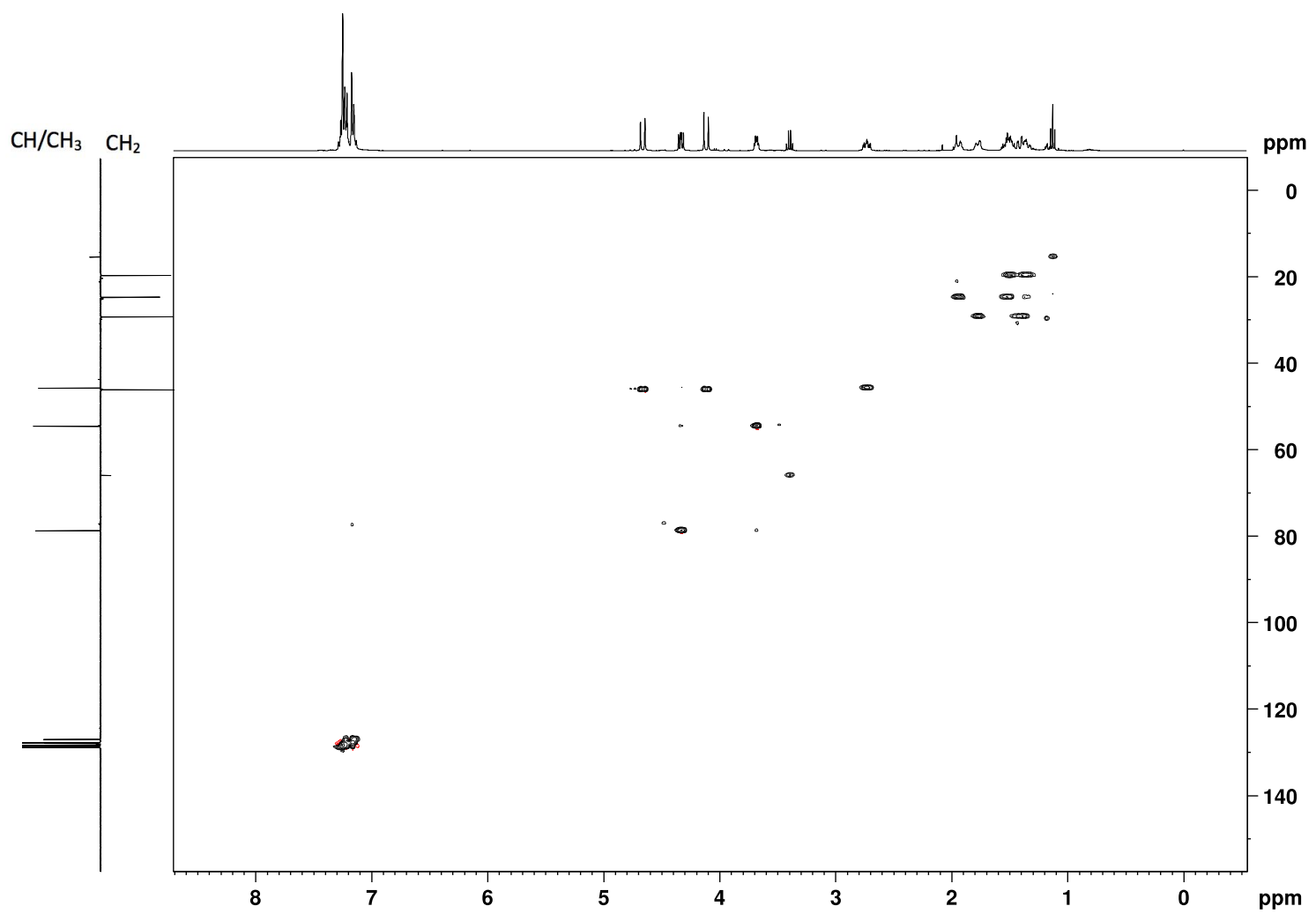

HSQC 2D-NMR (DEPT $135 /{ }^{1} \mathrm{H}, \mathrm{CDCl}_{3}$ ) - Zoom

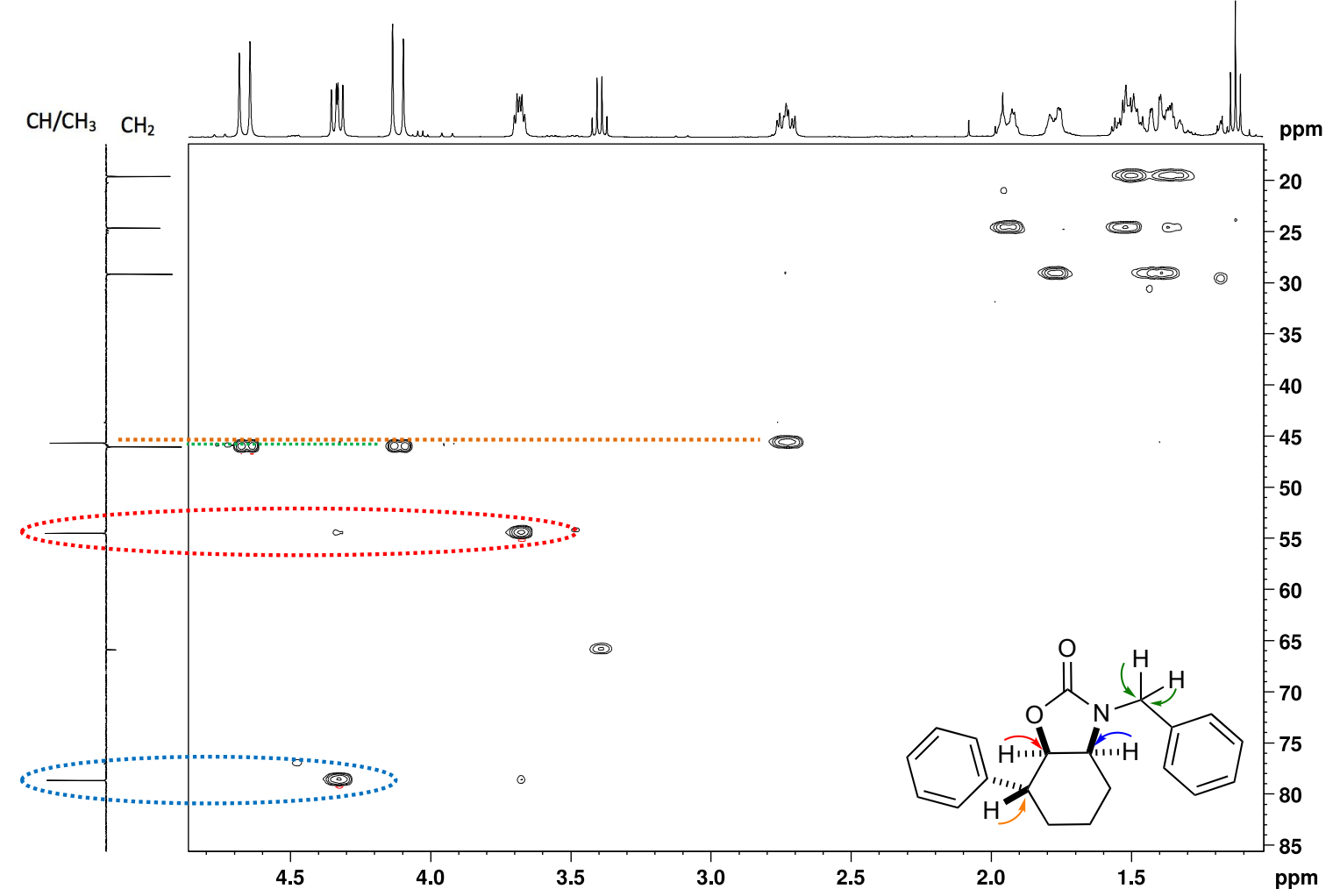


(士)-1-Benzyltetrahydro-1H,3H-pyrrolo[1,2-c]oxazol-3-one, 3k

${ }^{1} \mathrm{H} \mathrm{NMR}\left(400 \mathrm{MHz}, \mathrm{CDCl}_{3}\right)$

䲩

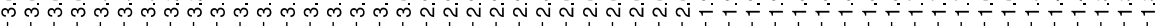<smiles>O=C1O[C@H](Cc2ccccc2)[C@@H]2CCCN12</smiles>

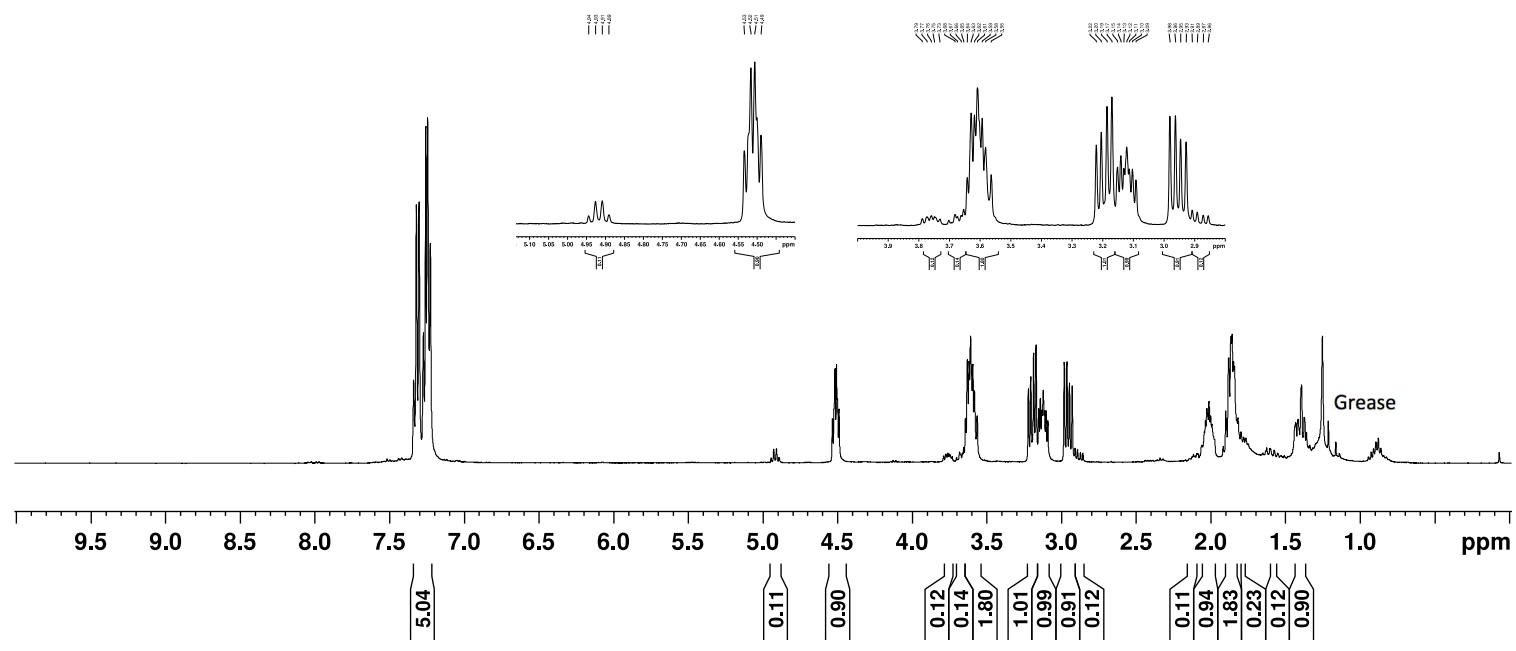

$\left.{ }^{13} \mathrm{C} \mathrm{NMR} \mathrm{(101} \mathrm{MHz,} \mathrm{CDCl}_{3}\right)$
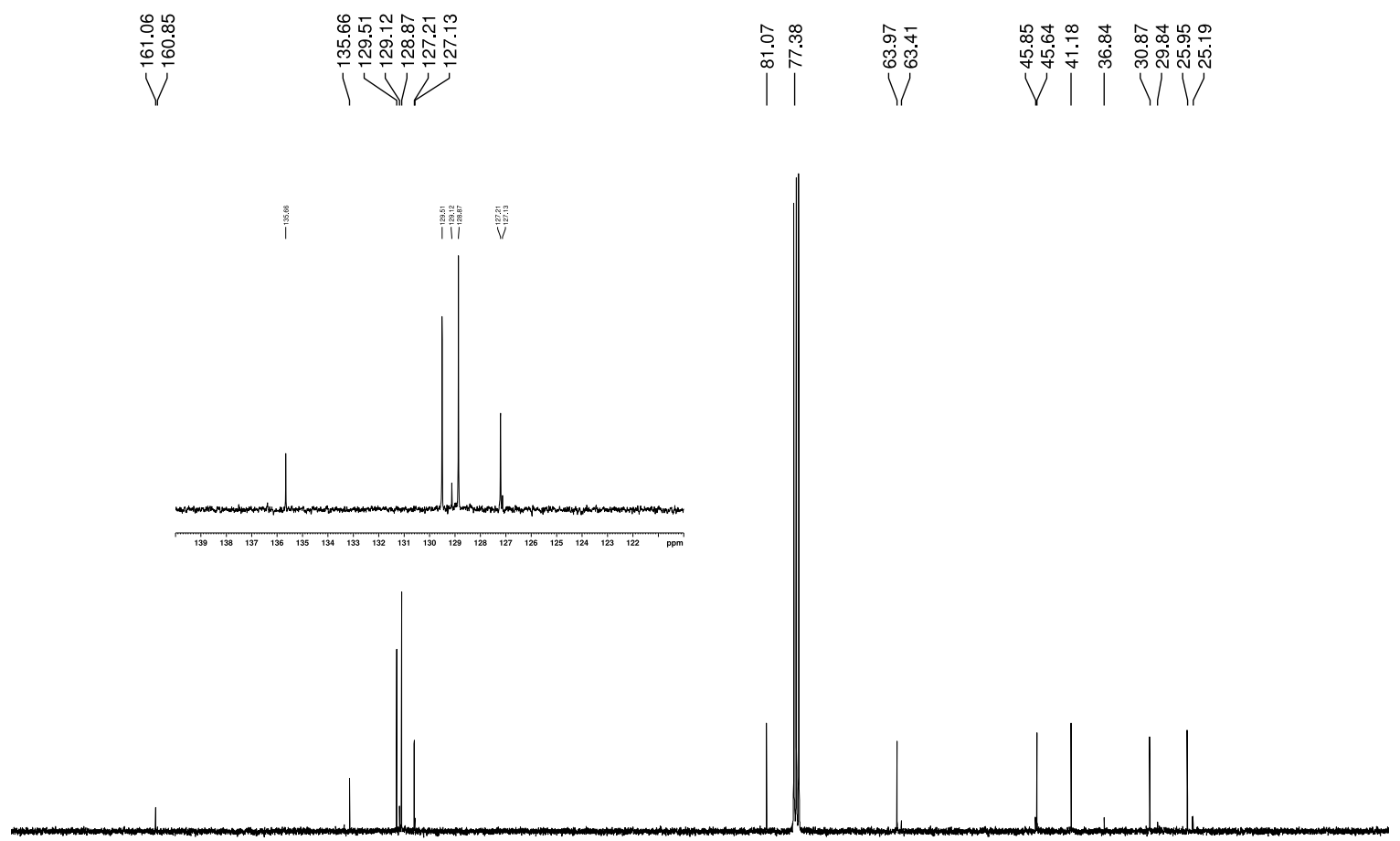

$170 \quad 160$

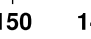

140130

120

110100

$90 \quad 80$

$70 \quad 60$

50

$40 \quad 30$

$2010 \mathrm{ppm}$ 
HSQC 2D-NMR (DEPT $135 /{ }^{1} \mathrm{H}, \mathrm{CDCl}_{3}$ )

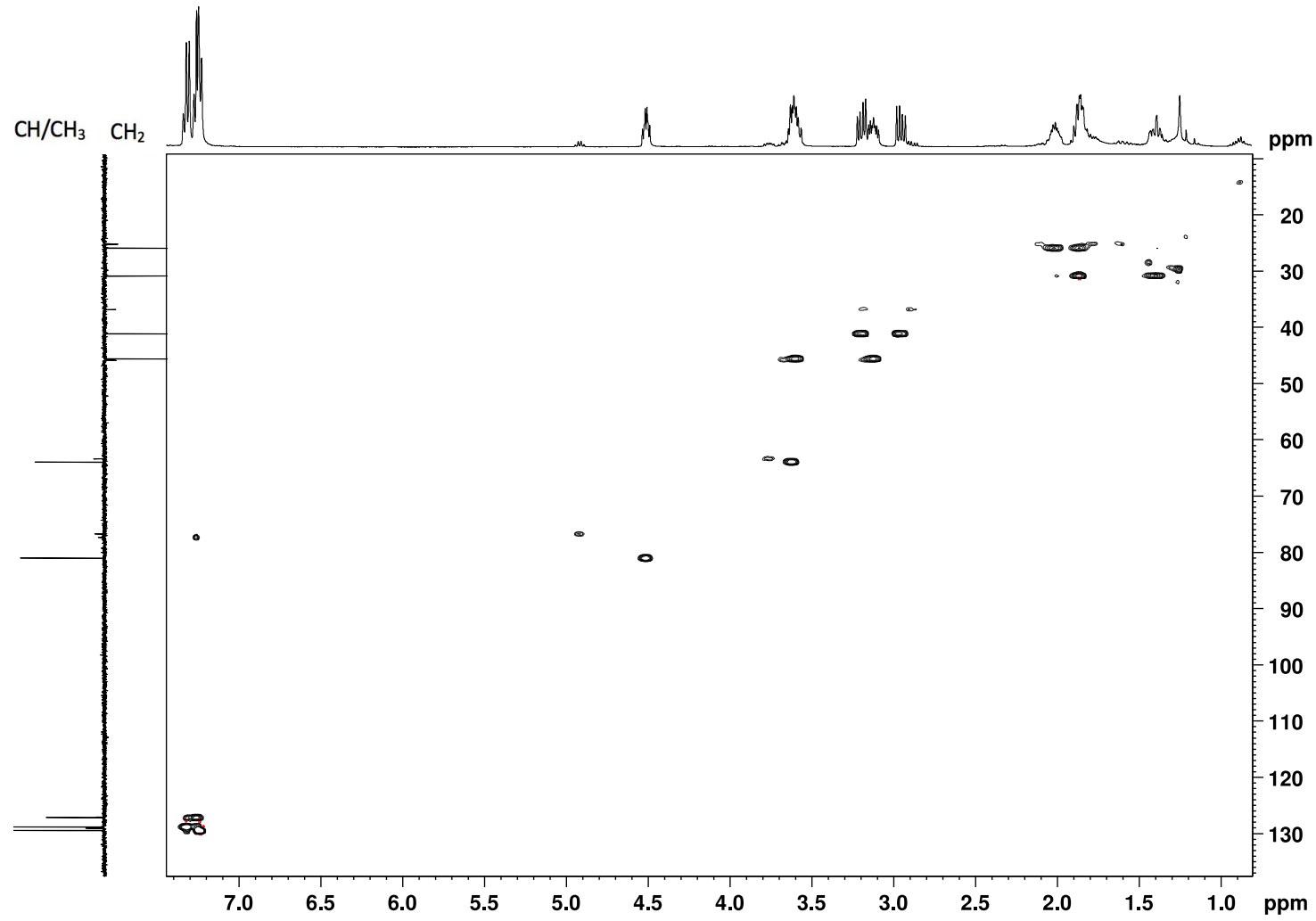

HSQC 2D-NMR (DEPT $135 /{ }^{1} \mathrm{H}, \mathrm{CDCl}_{3}$ ) - Zoom

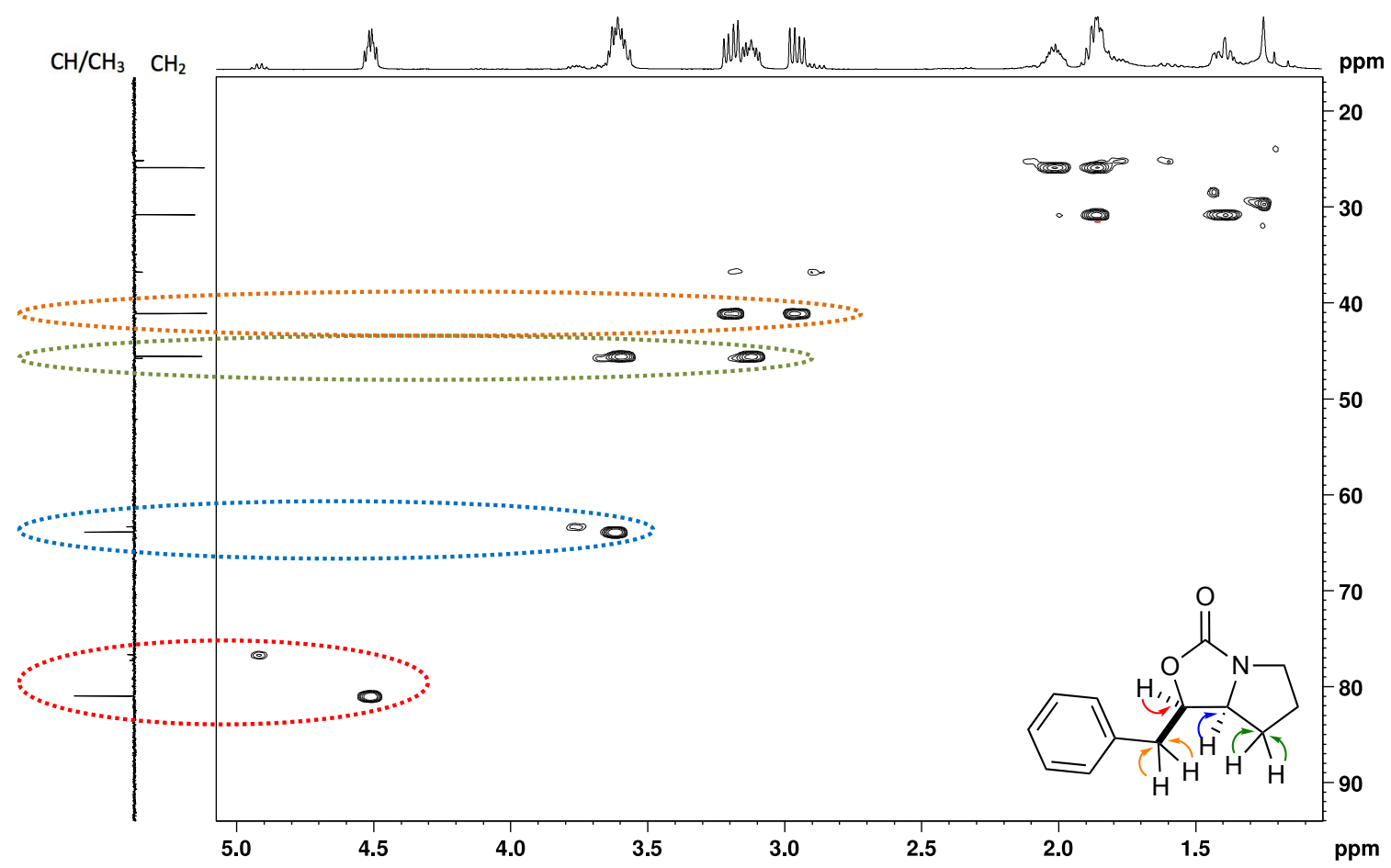


(士)-1-Benzylhexahydro-3H-oxazolo[3,4-a]pyridin-3-one, 31

${ }^{1} \mathrm{H} \mathrm{NMR}\left(400 \mathrm{MHz}, \mathrm{CDCl}_{3}\right)$

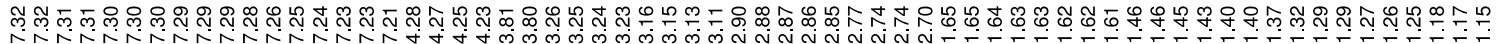<smiles>O=C1O[C@H](Cc2ccccc2)[C@H]2CCCCN12</smiles>

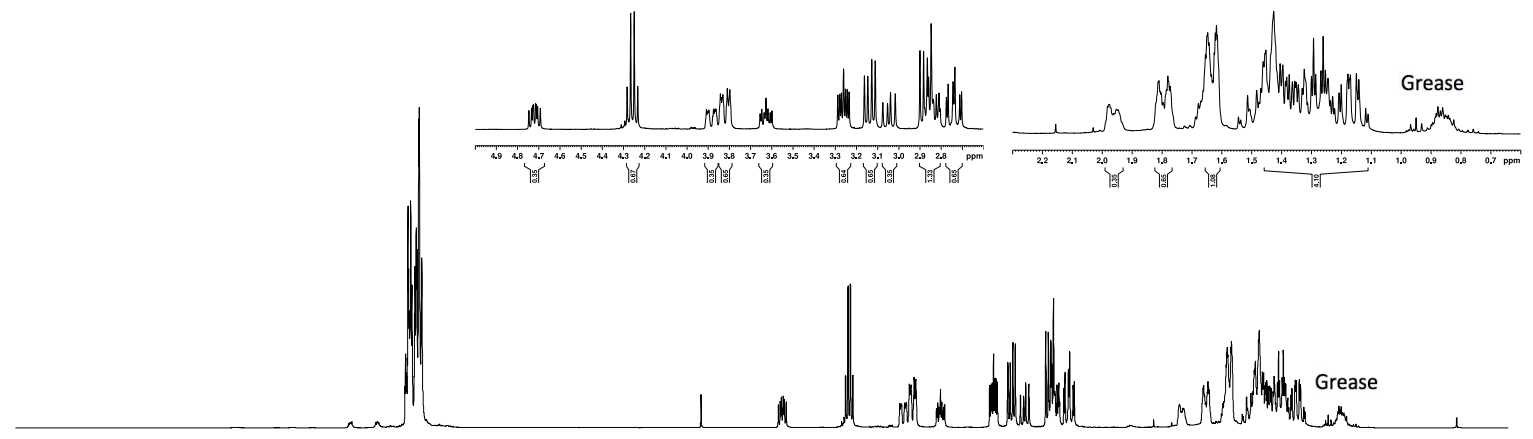

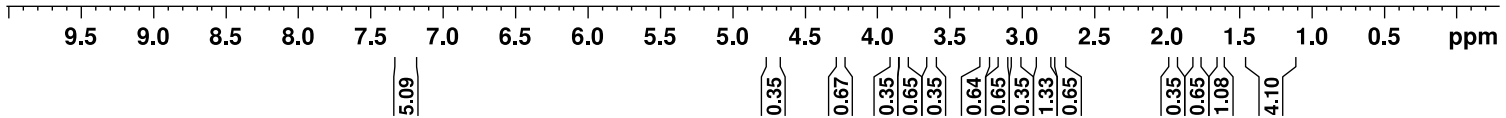

${ }^{13} \mathrm{C}$ NMR $\left(101 \mathrm{MHz}, \mathrm{CDCl}_{3}\right)$
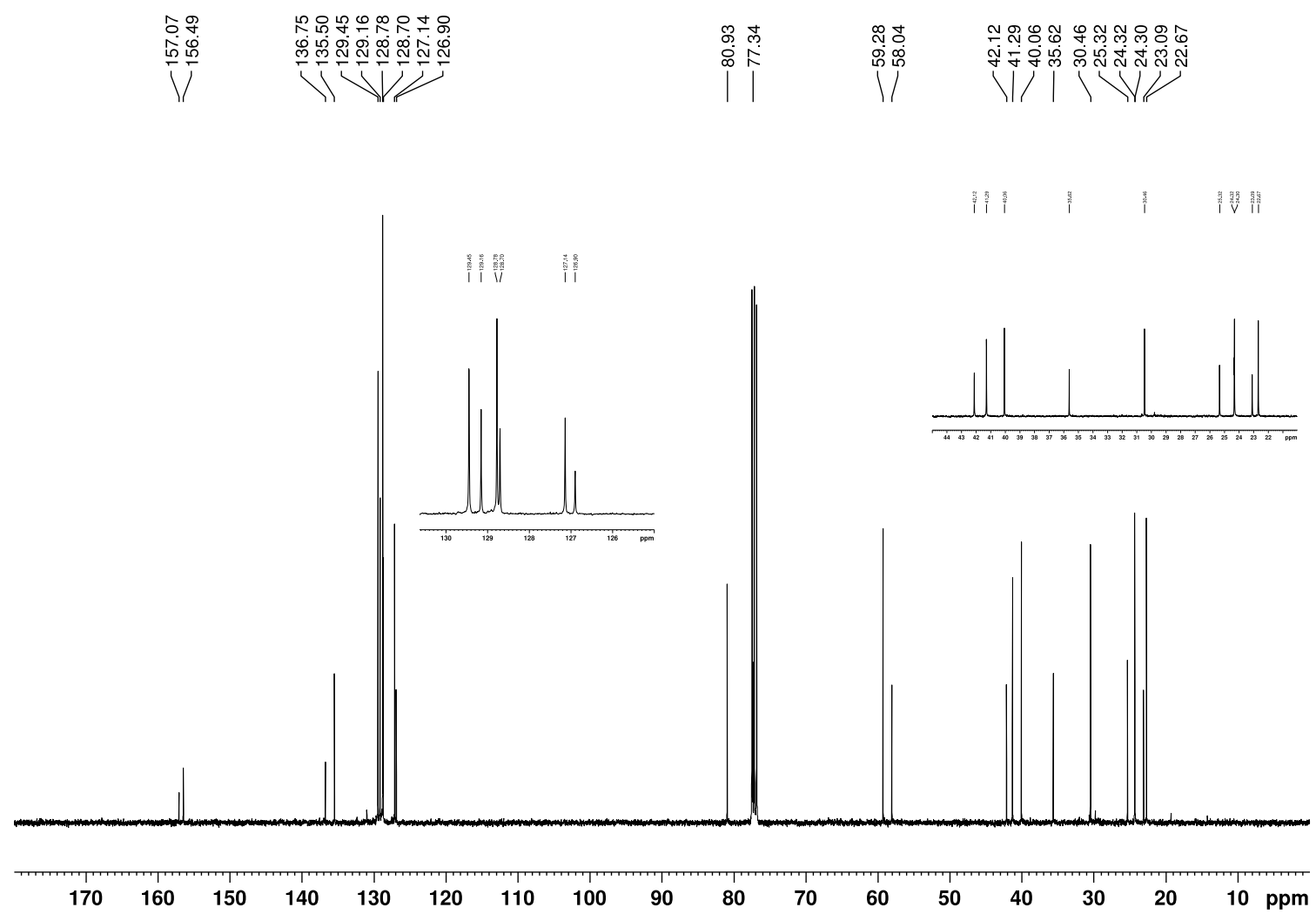
HSQC 2D-NMR (DEPT $135 /{ }^{1} \mathrm{H}, \mathrm{CDCl}_{3}$ )

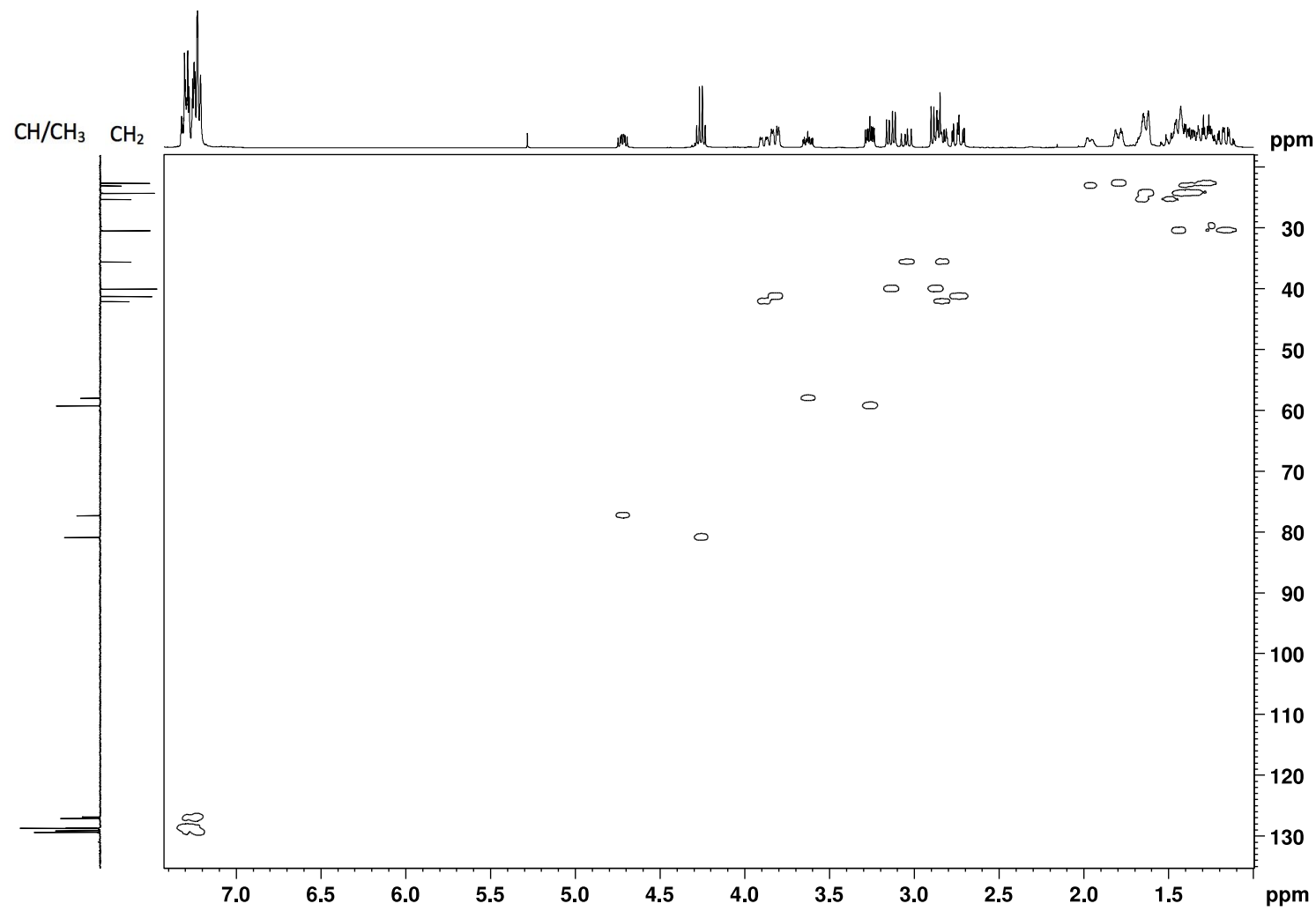

HSQC 2D-NMR (DEPT $135 /{ }^{1} \mathrm{H}, \mathrm{CDCl}_{3}$ ) - Zoom

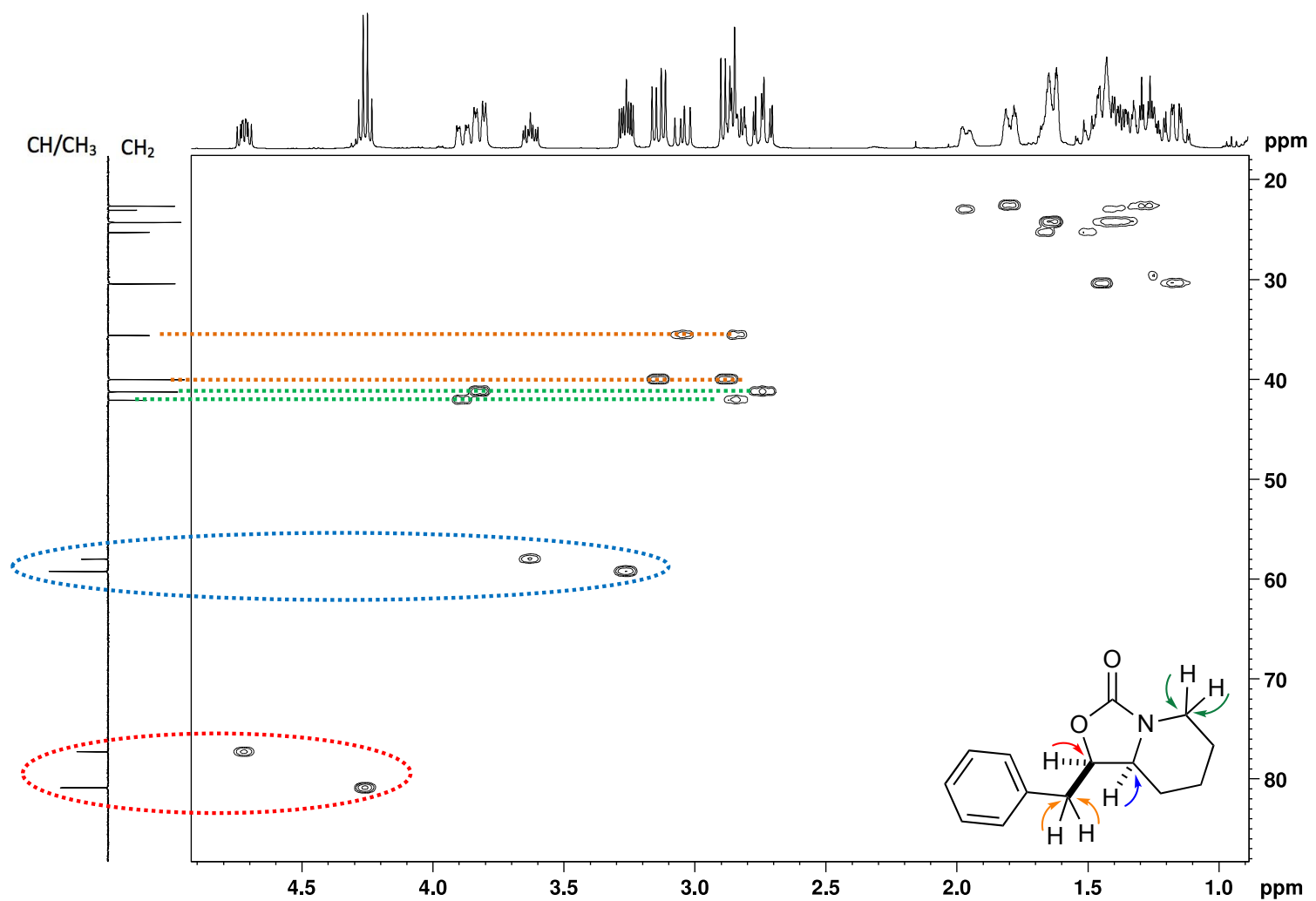


(士)-N-Benzyl-6-phenyl-1-oxa-3-azaspiro[4.5]decan-2-one, 3m

${ }^{1} \mathrm{H} \mathrm{NMR}\left(400 \mathrm{MHz}, \mathrm{CDCl}_{3}\right)$

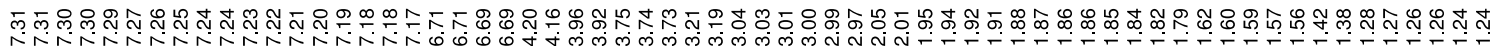
4 V

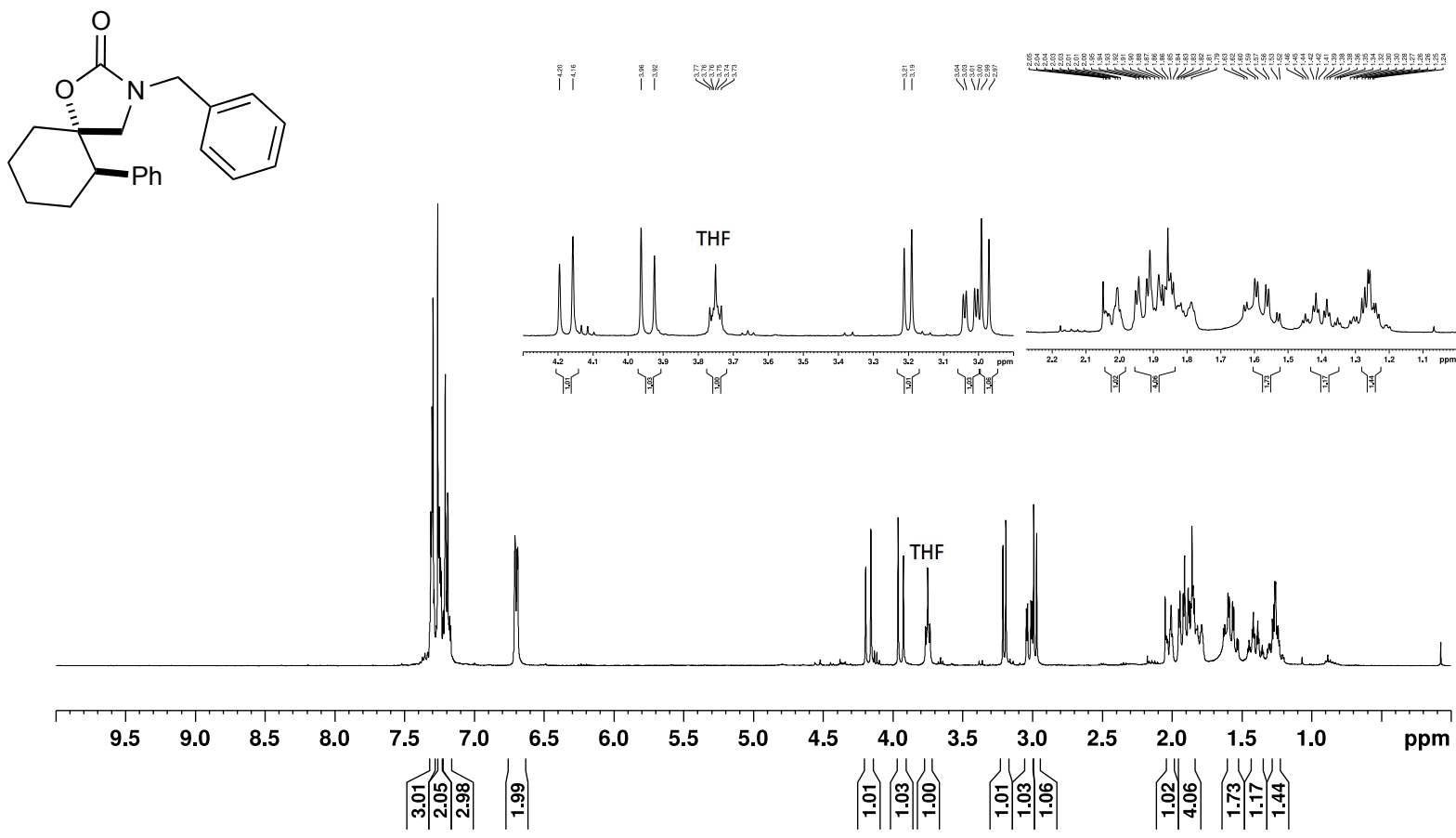

${ }^{13} \mathrm{C} \mathrm{NMR}\left(101 \mathrm{MHz}, \mathrm{CDCl}_{3}\right)$
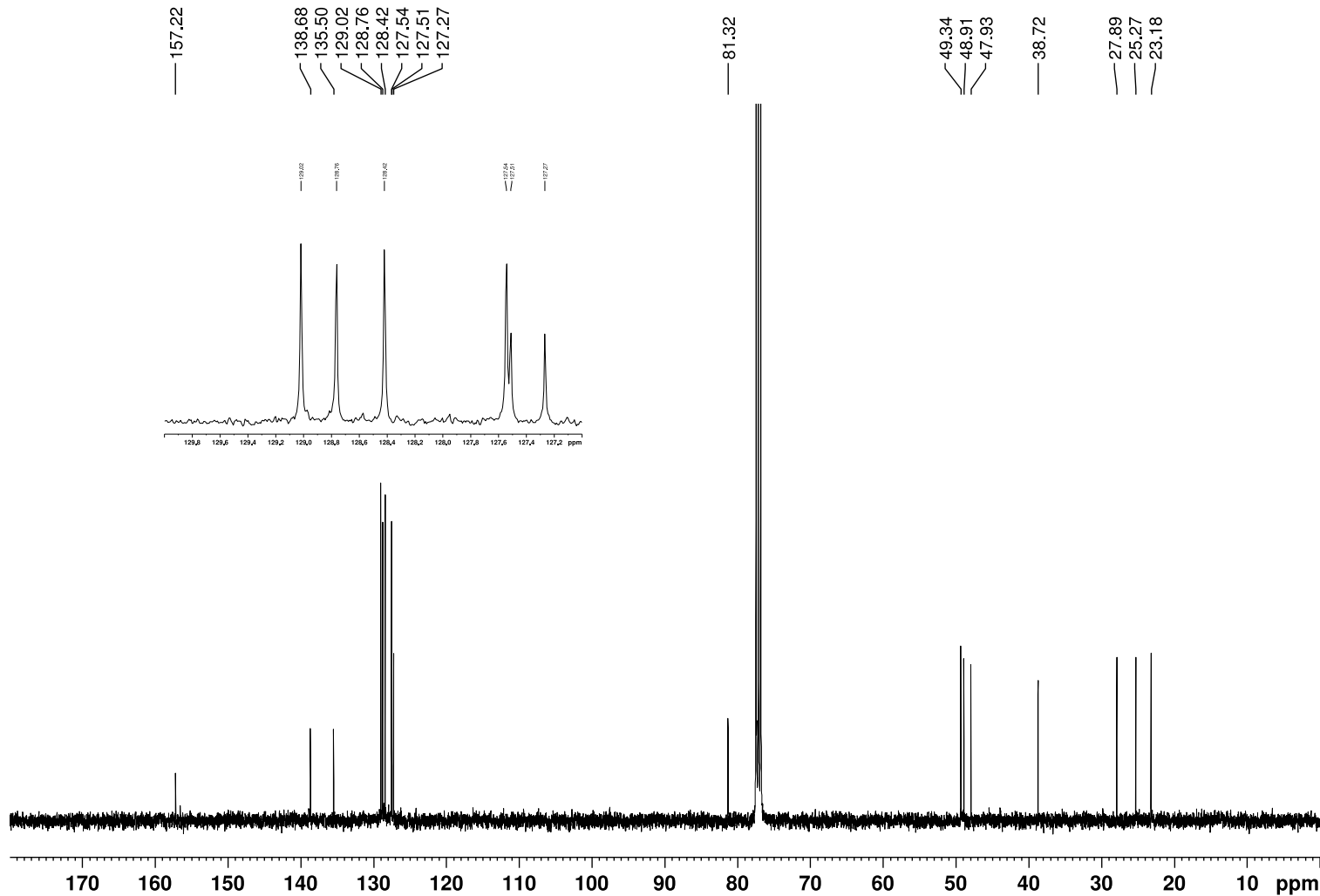
$(+)-(R)-1-[N-(R)-P h e n y l e t h y l a m i n o]-p r o p a n-2-o l, ~ 4$ ${ }^{1} \mathrm{H} \mathrm{NMR}\left(400 \mathrm{MHz}, \mathrm{CDCl}_{3}\right)$

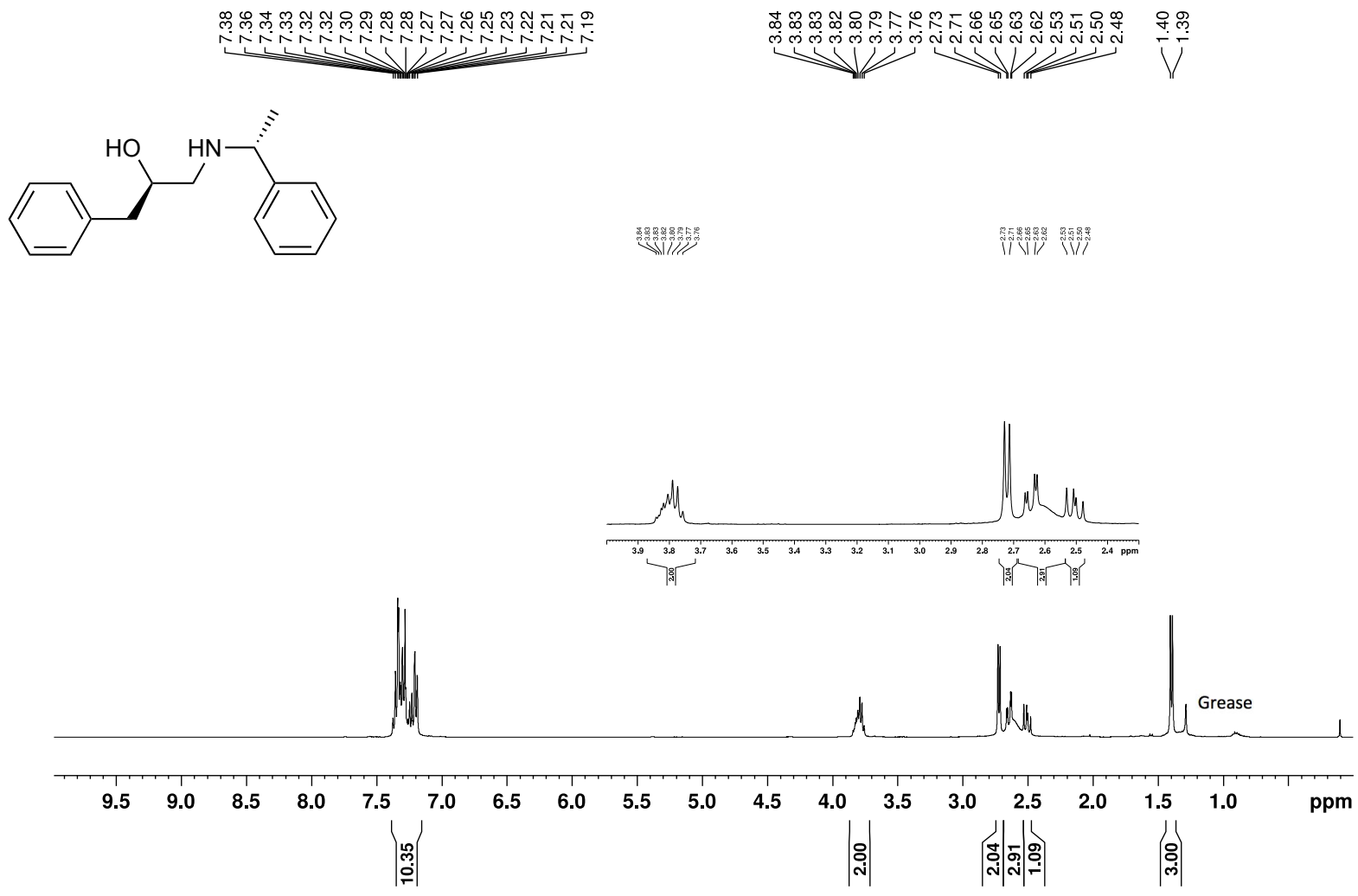

${ }^{13} \mathrm{C}$ NMR $\left(101 \mathrm{MHz}, \mathrm{CDCl}_{3}\right)$

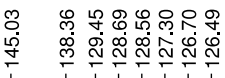

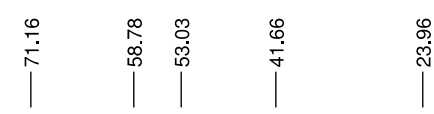

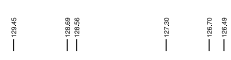
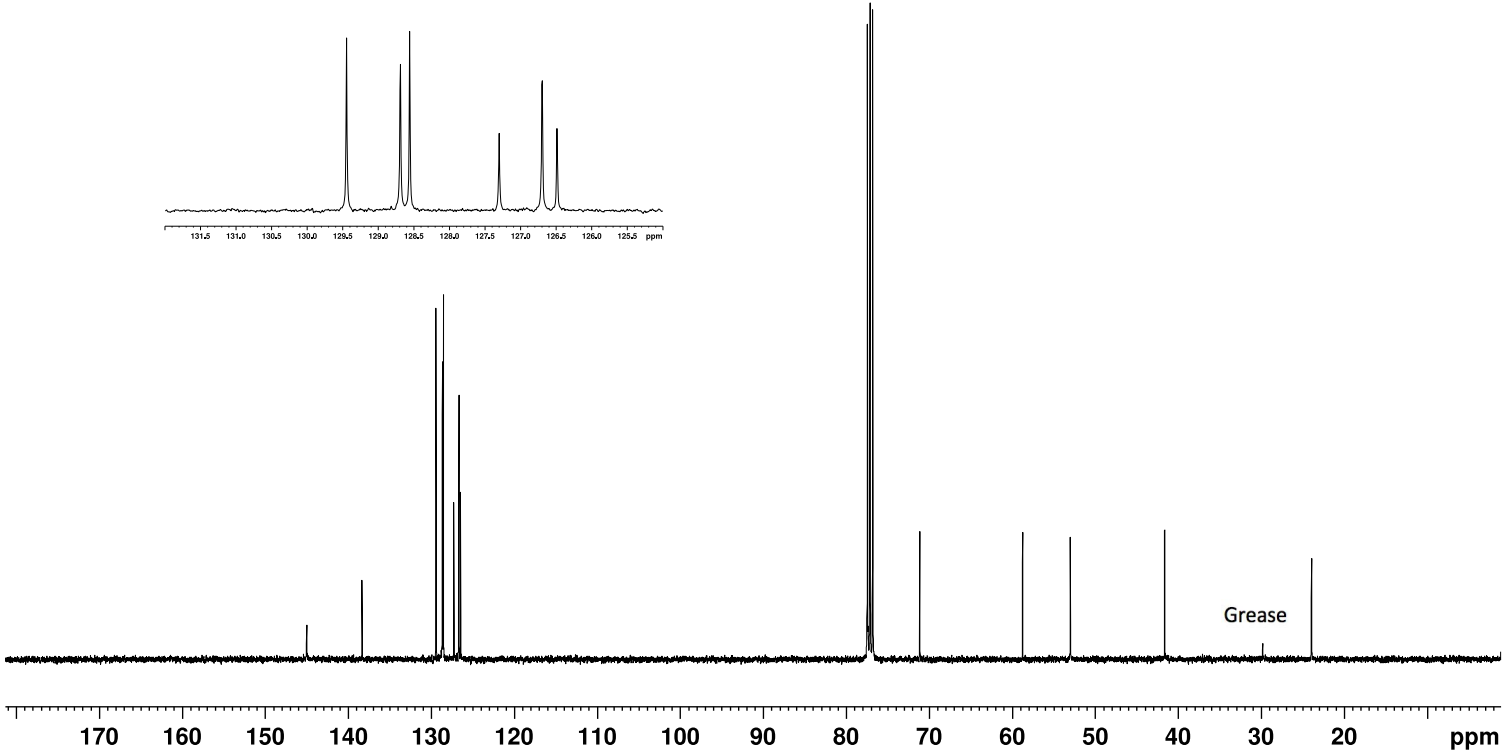
( \pm )-N-Benzyl-5-cyclopropylmethyl-oxazolidin-2-one, 3n

${ }^{1} \mathrm{H} \mathrm{NMR}\left(400 \mathrm{MHz}, \mathrm{CDCl}_{3}\right)$

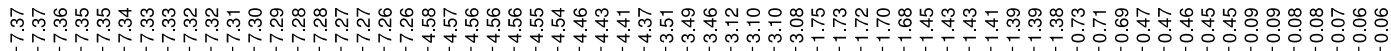

W

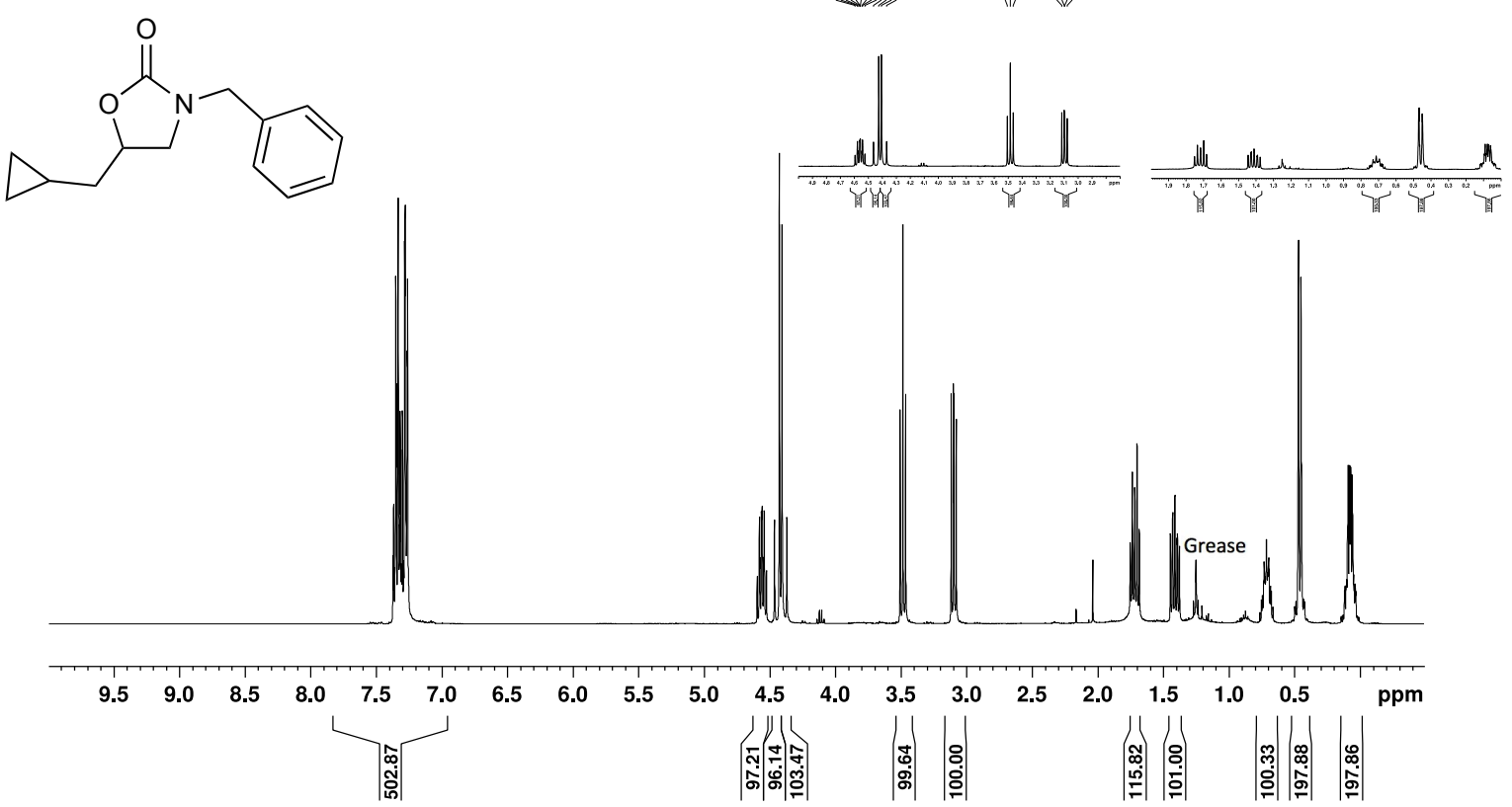

$\left.{ }^{13} \mathrm{C} \mathrm{NMR} \mathrm{(101} \mathrm{MHz,} \mathrm{CDCl}_{3}\right)$

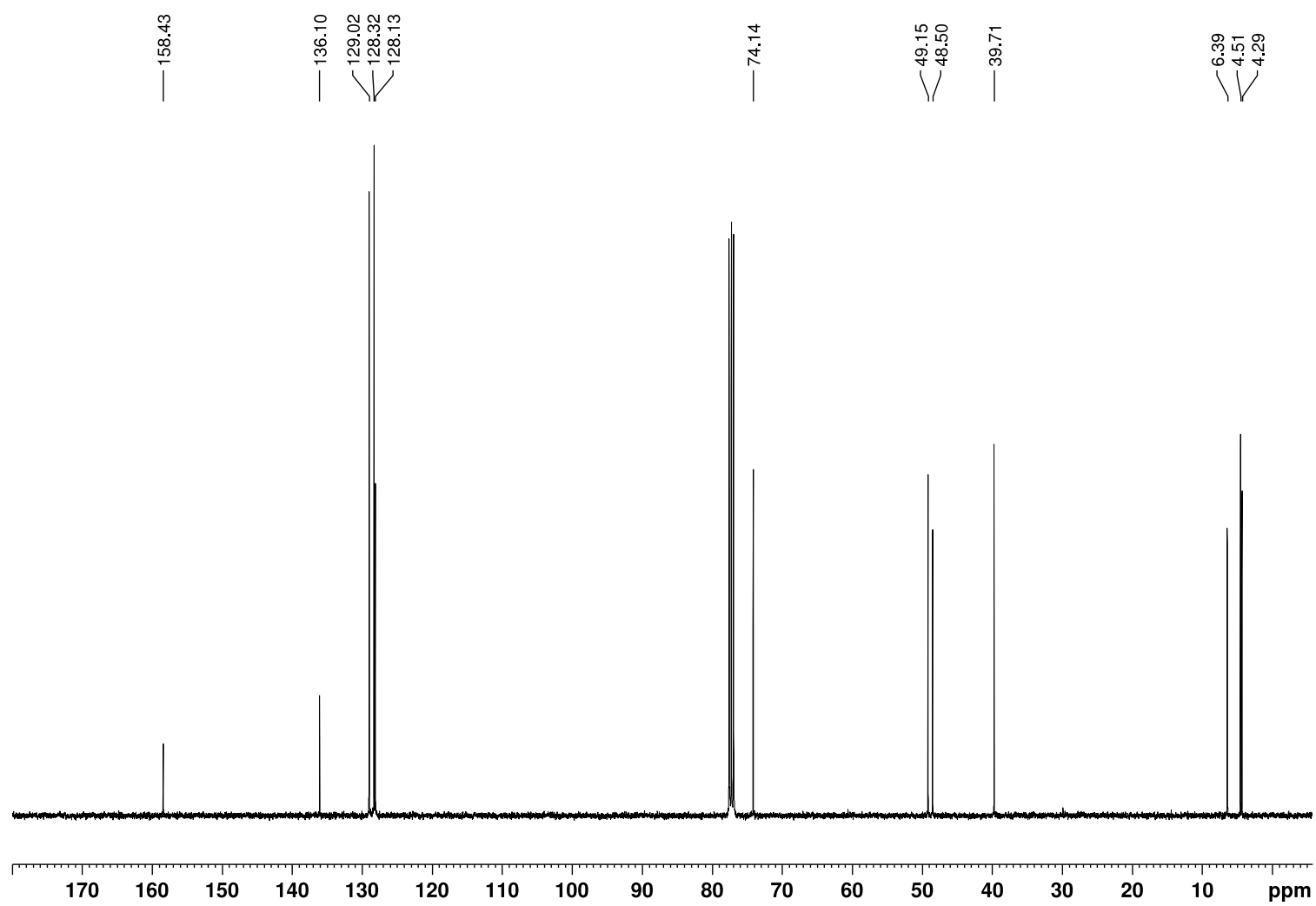

